\title{
Cerebral and cardiac signal monitoring in fetal sheep with hypoxic-ischemic encephalopathy
}

Citation for published version (APA):

Zwanenburg, A. A. (2015). Cerebral and cardiac signal monitoring in fetal sheep with hypoxic-ischemic encephalopathy. [Doctoral Thesis, Maastricht University]. Maastricht University. https://doi.org/10.26481/dis.20151119az

Document status and date:

Published: 01/01/2015

DOI:

10.26481/dis.20151119az

Document Version:

Publisher's PDF, also known as Version of record

\section{Please check the document version of this publication:}

- A submitted manuscript is the version of the article upon submission and before peer-review. There can be important differences between the submitted version and the official published version of record.

People interested in the research are advised to contact the author for the final version of the publication, or visit the DOI to the publisher's website.

- The final author version and the galley proof are versions of the publication after peer review.

- The final published version features the final layout of the paper including the volume, issue and page numbers.

Link to publication

\footnotetext{
General rights rights.

- You may freely distribute the URL identifying the publication in the public portal. please follow below link for the End User Agreement:

www.umlib.nl/taverne-license

Take down policy

If you believe that this document breaches copyright please contact us at:

repository@maastrichtuniversity.nl

providing details and we will investigate your claim.
}

Copyright and moral rights for the publications made accessible in the public portal are retained by the authors and/or other copyright owners and it is a condition of accessing publications that users recognise and abide by the legal requirements associated with these

- Users may download and print one copy of any publication from the public portal for the purpose of private study or research.

- You may not further distribute the material or use it for any profit-making activity or commercial gain

If the publication is distributed under the terms of Article $25 \mathrm{fa}$ of the Dutch Copyright Act, indicated by the "Taverne" license above, 


\title{
Cerebral and cardiac signal monitoring in fetal sheep with hypoxic-ischemic encephalopathy
}

\author{
Dissertation
}

by Alexander Adriaan Zwanenburg

to obtain the degree of Doctor at Maastricht University on the authority of the Rector Magnificus Prof. dr. L.L.G. Soete

in accordance with the decision by the Board of Deans to be defended, in public

on Thursday 19 November 2015, at 16.00 hours 


\section{Promotors}

Prof. dr. Tammo Delhaas

Prof. dr. Boris W. Kramer

\section{Co-promotors}

Dr. Peter Andriessen, Máxima Medical Centre Veldhoven

Dr. Tim G.A.M. Wolfs

\section{Assessment committee}

Prof. dr. Ralf L. M. Peeters (chair)

Prof. dr. Arend F. Bos, University Medical Centre Groningen

Dr. Carola van Pul, Máxima Medical Centre Veldhoven

Prof. dr. Uli Schotten

Prof. dr. Marc E. A. Spaanderman 


\section{Table of Contents}

Chapter 1:

Chapter 2:

Chapter 3:

Chapter 4:

Chapter 5:

Chapter 6:

Chapter 7:

Chapter 8:
General introduction 5

Detecting hypoxia with ST waveform analysis $\quad 15$

ECG markers for detecting hypoxia 29

Detection of short seizures $\quad 51$

Interhemispheric burst synchrony 71

Baroreceptor reflex function in HIE 81

Baroreceptor reflex function in HIE with stem cell treatment $\quad 97$

General discussion $\quad 117$

References $\quad 127$

Summary 139

Valorisation 143

Acknowledgements $\quad 149$

Curriculum vitae \& publication list 153

Propositions 157 



\section{General introduction}



Hypoxic-ischemic encephalopathy

An adequate fetal oxygen supply is essential for normal cerebral function and maturation. Low oxygen availability (hypoxia) and/or restriction of blood supply (ischemia) lead to brain injury. This brain injury is often referred to as hypoxic-ischemic encephalopathy (HIE). HIE is estimated to occur in 1.5 per 1000 live births ${ }^{1}$. In general, three clinical stages of increasingly severe neuronal loss are distinguished. These stages, referred to as 'mild', 'moderate' and 'severe' encephalopathy, are based on the infant's alertness, muscle tone, pupils, respiration and the presence of seizures ${ }^{2}$. HIE often has lifelong effects, ranging from mild motor or learning disabilities to cerebral palsy, blindness and premature death ${ }^{3}$. These effects lead to immense personal and societal burden, and it is estimated that neonatal encephalopathy causes 50 million disabilityadjusted life-years (DALY) globally ${ }^{4}$. In comparison, cancer was estimated to lead to 169 million DALY globally ${ }^{5}$.

Hypoxia-ischemia may be the result of several conditions. Examples of such conditions are a prolonged or repeated umbilical cord occlusion, placental rupture, placental insufficiency, placental ischemia, fetal infection and maternal disease. The neuronal damage resulting from hypoxia-ischemia develops in two phases ${ }^{6,7}$. The acute phase, when hypoxia-ischemia and acidosis is present, causes a degree of neuronal loss which depends on the length and severity of the hypoxic-ischemic period and the infant's metabolic reserves. As soon as the condition resulting in hypoxia-ischemia has been resolved, tissue is reperfused. Vital signs, such as heart rate, blood pressure and blood oxygen saturation return to normal. However, tissue reperfusion after hypoxiaischemia sets processes in motion that adversely affect neurons several hours after the incident. In general most permanent neuronal damage occurs within the period from 6 to 72 hours after start of reperfusion ${ }^{7}$. This period is characterised by seizure activity, cerebral cytotoxic edema, accumulation of excitotoxins in the brain, cerebral inflammatory processes, failure of cerebral mitochondrial activity and cell death ${ }^{7,8}$. The start of this subacute phase of neuronal damage signifies the point at which current treatments will fail to improve condition the infant. Because of this, the first 6 hours after reperfusion is considered to be a treatment window ${ }^{9}$.

Current treatment options for HIE are limited and often merely supportive. Nowadays, a standard treatment for term infants with moderate and severe HIE is therapeutic hypothermia, which is achieved through active body cooling. The infant's body temperature is lowered to $33^{\circ} \mathrm{C}$ and maintained at this temperature for 72 hours, although different settings for temperature and duration are being investigated as well ${ }^{9}$. Therapeutic hypothermia prevents apoptotic cell death, inhibits inflammation, reduces disruption of the blood brain barrier and increases neurotrophin levels and their receptors $^{8}$. Therapeutic hypothermia thus attenuates neuronal losses during the subacute phase of neuronal loss in term infants with moderate and severe grades of HIE. Additionally, hypothermia treatment reduces the incidence of long-term adverse outcomes ${ }^{10}$. However, hypothermia treatment is not successful in all infants with HIE. Recently, the Total Body Hypothermia for Neonatal Encephalopathy Trial (TOBY) reported the effect of hypothermia treatment on neurocognitive function of the participating children at 6 or 7 years old. In the TOBY trial 8 children needed to be treated in order to prevent 1 child from dying or having an IQ score $<85^{11}$. Also, hypothermia treatment for infants born preterm ( $<36$ weeks of gestation) is not widely 
implemented, due to suspected harmful effects of the therapy. Clinicians are reluctant to treat preterm infants with HIE, despite the fact that actual proof for the harmful effect of hypothermia treatment in preterm infants with HIE has not been established ${ }^{9}$.

Another issue in treating HIE is assessing the severity of the initial hypoxic-ischemic insult and determining treatment prognosis ${ }^{12}$. In other words, clinicians have to decide whether the initial hypoxic-ischemic insult was severe enough to warrant treatment and whether they are still in time to start treatment. It is sometimes unclear when the initial hypoxic-ischemic insult took place and how severe it was. Some infants develop hypoxia gradually, starting hours before birth, thus severely reducing the treatment window. Other infants suffer a hypoxic-ischemic insult even earlier and have already entered the subacute phase before birth. Again in other infants the initial hypoxic-ischemic insult might be so severe that the onset of the subacute phase takes places earlier ${ }^{13}$. Markers are thus needed to confirm the presence and assess severity of the initial hypoxicischemic insult and determine treatment prognosis. Many markers have been reviewed by Bennet et al. who came to the conclusion that although many markers show good correlations with outcomes, they only do so once the subacute phase has already started $^{12}$. Interpretation of these markers therefore cannot support clinicians in determining whether an infant can and should be treated. The issue becomes even more complicated in preterm infants. Many markers for HIE, such as muscle tone and EEG activity, that would indicate term infants with HIE are normal for preterm infants ${ }^{14-}$ ${ }^{17}$ and, hence, are less reliable in assessing HIE in this group of vulnerable patients.

Infants with HIE are kept under observation at the NICU for treatment and/or recovery. Their vital signs, such as heart rate, blood pressure and oxygen saturation, are monitored to identify acute risks to their well-being. Neuronal loss may be assessed at the anatomical level through MRI, but it may also be assessed at the level of cortical, subcortical and autonomic function. The latter commonly involves more advanced techniques than used for monitoring vital signs. Such functional analysis may lead to identification of threatening conditions which develop gradually and would be missed by short-term observations of vital signs and histological assessment of cerebral injuries.

Hence we will focus on two areas in this thesis (Figure 1.1): 1) early detection of hypoxia-ischemia to avoid or limit HIE through timely intervention, and 2) assessment of cortical, subcortical and autonomic function to assess HIE severity and to warn for conditions that may lead to further brain injury. In the above areas interpretation of physiological signals is necessary. In the work described in this thesis we analysed several signals that can be measured and monitored continuously. Notably, we analysed electrocardiographic (ECG), blood pressure (BP) and electroencephalographic (EEG) signals to examine their clinical implications in HIE.

Interpreting signals through data-driven algorithms

Signals are evaluated through interpretation of one or more characteristic features. The features provide clinicians with information necessary to make a well-founded decision. For example, identifying characteristic features associated with seizure activity will help the clinician decide upon starting an anti-convulsant therapy, or in the case of suspected HIE help assess the HIE grade. ECG, BP and EEG monitoring produces 
Early detection of conditions

leading to HIE

Chapter 2 - 3
Assessment of cortical, subcortical

and autonomic function

Chapter 4 - 7

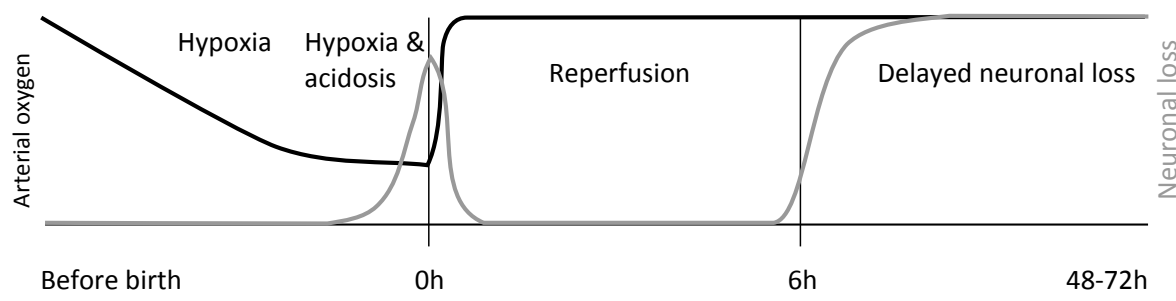

Figure 1.1: Course of brain injury in hypoxic ischemic encephalopathy and thesis contents. Prolonged hypoxia (potentially through ischemia- a disruption in blood flow) can lead to the development of acidosis and concomitant brain injury. Reperfusion starts after the condition leading to hypoxia has been resolved. Reperfusion, in absence of treatment, sets in motion processes which may lead to a second stage of neuronal loss, even in the absence of hypoxia. The second stage of neuronal loss starts at around 6 hours after reperfusion and lasts up to 72 hours. Early detection of conditions leading to HIE can help limit or avoid HIE by allowing early intervention, and is considered in chapters 2-3. Assessment of cortical, subcortical and autonomic function can help establish HIE severity and identify risks for additional brain injury, and is considered in chapters 4-7.

complex, longitudinal signals. Computerised algorithms may aid in the analysis and interpretation of signals. In addition, signal analysis by a clinical decision support algorithm may reduces the intra- and interobserver variability inherent to human observers $^{18,19}$. Furthermore, it may reduce the time necessary for clinicians to interpret the recordings ${ }^{20}$.

Clinical decision support algorithms ideally have a strong predictive value, i.e. they interpret markers and produce the right decision. A set of rules is necessary to translate input features to an output decision. Such a rule set can be created manually by human investigators or device manufacturers, or can be data-driven. Many current clinical algorithms are still based on manual rule sets. Though the latter rule sets have the advantage of being more easily understood by clinicians, the evidence for such rule sets is at times vague or altogether lacking. This can cause algorithms to fail on new clinical data, even though the rule set did apply for the studies it was developed on ${ }^{21}$. Ideally clinical computer algorithms are based on data-driven rule sets ${ }^{22}$. Such a data-driven approach is called machine learning. In machine learning features are assigned to a class by a classifier. The classifier is the rule set that is trained using feature data for which the class is known. An example from voice recognition can elucidate this process. In this example the aim of voice recognition is the automatic transcription of a conversation. Audio signal recorded by a microphone needs to be translated to written script by an algorithm. Investigators, through trial and error, manage to define features which adequately describe the audio signal, such as the frequency spectrum of the audio signal. These features are then interpreted by the classifier in the voice recognition software and the correct word is transcribed. Before this can happen, the 
classifier needs to learn which feature values correspond to which vowel sound. Of course, exact pronunciation of vowels differs between individuals. Multiple individuals will therefore first need to produce the vowel sound to allow the classifier to determine which range and combination of feature values correspond to the vowel produced.

In the example above investigators defined features that adequately capture the audio signal and allow it to be interpreted. In general, features need to be sensitive and specific for the problem being examined so that they can be effectively interpreted by a computer algorithm and used as markers. Concretely, to prevent or limit hypoxicischemic brain injury by early intervention, potential markers should provide an early alarm for conditions that could lead to HIE. Likewise, assessment of cortical, subcortical and autonomic function to establish HIE severity and to identify adverse conditions requires markers that serve as a surrogate for cortical, subcortical and autonomic function. The aim of this thesis is to evaluate current markers and to identify new markers that can be used by clinical decision support systems.

\section{An animal model for severe hypoxia-ischemia}

Signals in this thesis are recorded in humans, but also in animals because the reproducible environment offered by an animal model was often required. Perinatal diseases, including HIE, have been very successfully studied in sheep ${ }^{23-29}$. Back et al. reviewed the applicability of fetal ovine sheep as a model for white matter injury (WMI) in the preterm infant ${ }^{30}$. As WMI is the major form of $\mathrm{HIE}^{3}$, many of their comments are applicable to the fetal ovine model for HIE. First, the fetal preterm model is viable even after instrumentation, i.e. mortality and morbidity in the model is low. Second, the developmental biology and the physiology of the fetal lamb closely resemble the human situation, including hemodynamics and neurodevelopment which are relevant to HIE.

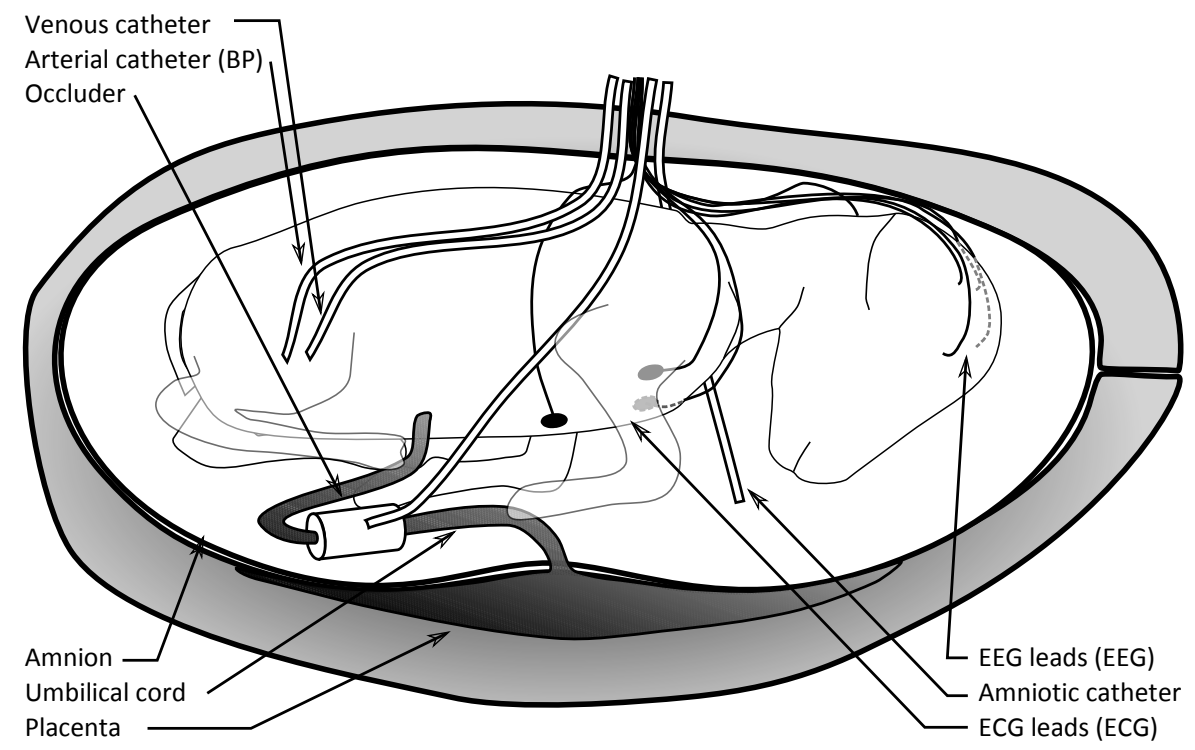

Figure 1.2: The fetal ovine model was chronically instrumented to measure EEG, blood pressure and ECG signals. A standardised period of hypoxia-ischemia was achieved through inflating and deflating the umbilical cord occluder. 
Cerebral white matter is abundant and anatomically similar to that of humans.

Myelination in the ovine fetus starts prenatally, as it does in the human fetus. Third, histopathology of the hypoxic-ischemic fetal ovine brain is comparable to that of humans as mostly white matter is affected. Fourth, fetal sheep are large animals, which allows for instrumentation for continuous measurement of electrical activity of the heart, blood pressure and electrical cortical activity. Therefore the preterm ovine fetus is an important and useful model to study clinically relevant $\mathrm{HIE}$.

The ovine fetus was chronically instrumented for ECG, EEG and BP measurements at an age of 102 days of gestation (Figure 1.2), which is equivalent to 30-32 weeks of gestation in humans. At 106 days of age, an umbilical cord occluder was inflated, thus halting flow of oxygen-rich blood from the placenta to the fetus. After a period of 25 minutes, the occluder was deflated and blood could flow again. During umbilical cord occlusion the fetus becomes hypoxic, blood $\mathrm{pH}$ decreases and blood carbon dioxide content increases, setting in motion processes that will lead to the development of severe HIE.

\section{Chapter 2: Detecting hypoxia with ST waveform analysis}

Fetuses are regularly monitored for fetal hypoxic distress during birth. Hypoxia affects heart rate patterns and fetal heart rate responses to uterine contractions. To detect hypoxia and acidosis, fetal heart rate and uterine contractions are monitored using a technique called cardiotocography (CTG). Remarkably, CTG did not significantly reduce adverse clinical outcomes, but did lead to an increases of emergency caesarean sections ${ }^{31}$.

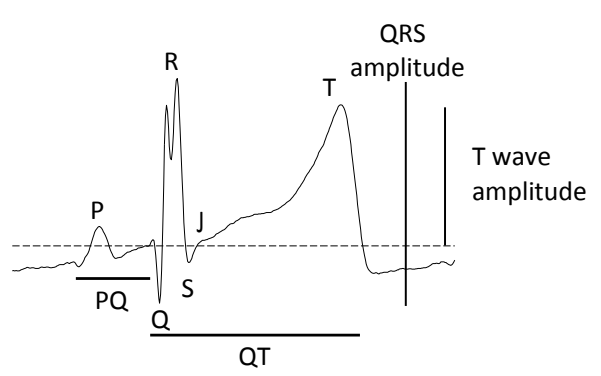
Hence additional information to assess developing fetal hypoxia is required. Hypoxia-ischemia inhibits aerobic glycolysis-a process necessary for

Figure 1.3: Fetal ECG with characteristic points. The $P$ wave is associated with atrial activation. The QRS complex is associated with ventricular activation, and the $T$ wave with ventricular repolarisation.

maintaining myocardial metabolism. Ventricular repolarisation is an energy-intensive process and gives rise to the ST waveform of the ECG, see Figure 1.3. Hypoxia-ischemia thus affects the shape of the ST waveform of the ECG ${ }^{32-34}$. ST waveform analysis (STAN) by means of the STAN device, was introduced in many clinics to provide information in addition to CTG to reduce the number of adverse outcomes and reduce the number of caesarean sections. Unexpectedly, introduction of the STAN device in clinical practice did neither reduce most adverse outcomes nor reduce the number of unnecessary caesarean sections $\mathrm{s}^{35,36}$. In the second chapter we investigated whether the device implementation of STAN might be a cause for the discrepancy between laboratory findings and clinical practice. To this end, we analysed ECG recordings from our standardised fetal model using a clinical STAN device and analysed the generated alarms. 


\section{Chapter 3: ECG markers for detecting hypoxia}

As meta-analyses have demonstrated that STAN appears not to be clinically useful, other markers for hypoxia-ischemia in addition to CTG are needed. ECG-based markers can be divided into interval and waveform-based markers. The markers used by STAN are examples of the latter, as they assess the shape of the ST waveform. Interval-based markers are based on the timing of characteristic points in the ECG. In contrast to ECG waveform-based markers, interval-based markers have the advantage of being less dependent on changes in projection angle of the electrical heart vector. Multiple markers for hypoxia, aside from those in STAN, have been proposed, based on fetal heart rate, atrioventricular delay and ventricular activation and repolarisation ${ }^{37-41}$. Direct comparison of the reported effectiveness of markers in detecting hypoxiaischemia is impeded by methodological differences between the studies. In the third chapter we used ECG recordings measured within the standardised animal model to investigate which ECG-based markers have the potential to detect the presence of hypoxia-ischemia in the fetus, and to determine possible practical differences between interval-based and waveform-based markers.

\section{Chapter 4: Detection of short seizures}

Cases of mild and moderate HIE are not easily distinguished in preterm infants because several discriminating characteristics for HIE grading are normal for prematurity. Surrogate markers for cortical, subcortical and autonomic function could aid in assessment of HIE severity in preterm infants as well as in observation of potential adverse conditions. One aspect of normal cortical function is the absence of seizures. Seizure activity by itself can adversely affect the developing brain and predispose the infant to recurrent seizure episodes at later age ${ }^{42,43}$. EEG is needed to establish seizure activity because seizures in preterm and term infants often do not have clinical correlates, such as involuntary muscle contractions ${ }^{18}$. Computer-assisted EEG assessment could aid clinicians in determining seizure presence, because EEG is difficult to interpret ${ }^{20,44,45}$. Several computer algorithms for seizure detection have been tested, which were based on either manually defined rule sets ${ }^{46-48}$ or on classifiers trained through machine learning ${ }^{49-51}$. In preterm infants computer-assisted detection of seizures is complicated by the fact that in preterm infants electrographic seizures are often short, i.e. less than 1 minute in duration ${ }^{45,49,52}$. An electrographic seizure can visually be divided in roughly three sections, namely onset, middle and end. Welltrained classifiers can most reliably assess the middle section which is relatively stable and contains pronounced seizure activity. However, in short seizures this middle section can be short in duration, if not absent, which hampers detection. Both onset and end of seizures are distinct transitions from and to non-seizure EEG background, but current algorithms do not incorporate these transitions for increasing detection performance. Hence, in the fourth chapter we described and tested a new marker which takes seizure onset and end into account.

\section{Chapter 5: Interhemispheric burst synchrony}

An aspect of normal subcortical function is synchronous EEG activity between both hemispheres, originating through thalamocortical driving and/or projection through the corpus callosum ${ }^{53,54}$. The preterm EEG is characterised by intermittent activity, called 
bursts, and quiet periods, called interburst intervals ${ }^{17}$. Asynchronous burst activity between hemispheres in the EEG indicates the presence of brain lesions ${ }^{55-57}$. Unlike the presence of seizure activity ${ }^{58}$ and other markers for $\mathrm{HIE}^{12}$, interhemispheric burst synchrony might already be affected by neuronal loss in the acute phase. This marker may thus be useful to assess the level of brain injury already present and may be used to create a treatment prognosis before the subacute phase starts.

The question whether burst synchrony is affected by maturation is unresolved, as some investigators did report a maturational effect ${ }^{56}$, and others did not ${ }^{59}$. It is important to know to what extent burst synchrony is affected by maturation because the effect of maturation and the effect of HIE may interfere, i.e. a preterm infant of 36 weeks' gestation with HIE might well show a level of burst synchrony comparable to an otherwise healthy preterm infant of 31 weeks. This would affect the choice of which particular machine learning algorithm should be used for assessing subcortical function. The studies of Lombroso and Anderson relied on manual analysis of burst synchrony in the EEG, which might have introduced errors or biases. Criteria for assessing interhemispheric burst synchrony are different as well, which might have contributed to the difference in findings. Thus, to reduce errors and biases and to assess to what extent a maturational effect is present in the EEG of healthy preterm infants, we used a computer algorithm to determine interhemispheric burst synchrony.

\section{Chapter 6: Baroreceptor reflex function in HIE}

In the sixth and seventh chapters we studied the baroreceptor reflex associated cardiovascular fluctuations in HIE. This autonomic system reflex mechanism ensures that mean arterial blood pressure is maintained at a constant level. The baroreceptors are pressure-sensing cells mainly located in the carotid sinuses and the aortic arch. Baroreceptors monitor changes in blood pressure and relay these to several interacting nuclei in the brain stem. Through a complex feedback mechanism the baroreceptor reflex buffers short term changes in blood pressure by adapting systemic vascular resistance, myocardial contractility and heart rate. HIE could affect the baroreceptor reflex function through neuronal loss in these brain stem regions. An impaired baroreceptor reflex may cause increased risk for developing additional brain injury ${ }^{60,61}$ by exposing the vulnerable developing cerebral vascular network to large fluctuations in blood pressure. Fluctuations associated with high blood pressure may disrupt the cerebral capillaries in the germinal matrix ${ }^{62}$. Otherwise, periods of low blood pressure may cause localised hypoxia-ischemia of the watershed areas ${ }^{62}$. If the baroreceptor reflex is adversely affected by HIE and if its suppression causes an additional risk for brain injury, it should be monitored in infants with HIE. Before the barorecepter reflex function can be used as a marker for increased brain injury, several questions need to be investigated. In the sixth chapter we investigated whether severe HIE affects baroreceptor reflex function in the ovine fetal model.

\section{Chapter 7: Baroreceptor reflex function in HIE with stem cell treatment}

The fetal brain is exposed to large fluctuations in blood pressure if baroreceptor reflex function is impaired by HIE, which may increase risk for developing additional brain injury. Treatments should aim to preserve baroreceptor reflex function in severe HIE. In the seventh chapter we studied the operation of the baroreceptor reflex after 
HIE in the presence and absence of stem cell therapy. Stem cell therapy has not been introduced in the clinic as a treatment for HIE, but is undergoing pre-clinical investigations within the context of stroke ${ }^{63}$, traumatic brain injury ${ }^{64,65}$ and neonatal hypoxia-ischemia ${ }^{66}$, among others. Stem cell therapy could help reduce neuronal loss, or the impact thereof, within the autonomic centres in the brain stem and thus preserve baroreceptor reflex function. In chapter seven we investigated whether stem cell therapy can mitigate or limit loss of baroreceptor reflex function after severe hypoxiaischemia.

Chapter 8: General discussion

The thesis ends with a chapter containing a general discussion of the main findings in the previous chapters and their broader clinical implications. 


\section{Detecting hypoxia with ST waveform analysis}

Submitted:

Zwanenburg, A., Andriessen, P., Vullings, R., Hermans, B. J. M., Niemarkt, H. J., Jellema, R. K., Ophelders, D. R. M. G., Wolfs, T. G. A. M., Kramer, B. W. \& Delhaas, T. Fetal ECG ST waveform analysis fails to detect hypoxic conditions in an ovine model. BJOG (2015) 



\section{Abstract}

Background Changes in ST waveform of the fetal ECG are associated with hypoxic distress. Because meta-analyses of clinical trials showed inconclusive results for ST waveform analysis (STAN) in detecting fetal hypoxic conditions, we hypothesized that the clinical device for STAN is unable to discriminate between normoxic and hypoxic conditions.

Methods 8 fetal lambs were exposed to 25 minutes of hypoxia by a transient umbilical cord occlusion (UCO). ECG was recorded during the period starting 4 hours before until 4 hours after UCO. ECG recordings were analyzed using a clinical STAN device. Alarms produced by the device were examined to assess its potential to distinguish between normoxic and hypoxic state.

Results 25 minutes of UCO resulted in hypoxia and acidosis. Whereas STAN produced many false alarms in the normoxic baseline period (low PPV of max 32\%), it produced few alarms during UCO (low alarm sensitivity of max 11\%). Remarkably, STAN did not generate any alarms during UCO for two out of eight fetuses. Low values for Matthews correlation coefficients for STAN alarms (ranging between -0.28 and 0.12 ) indicate that the STAN device could not discriminate between normoxic and hypoxic conditions of the fetus.

Conclusion STAN could not detect hypoxia-ischemia after complete UCO in a standardized model for severe hypoxia-ischemia. Whereas alarms were generally abundant during the normoxic baseline period, they were mostly absent during the UCO period. Poor performance of STAN in assessing the normoxic/hypoxic state of a fetus with severe hypoxia-ischemia might explain limited effectiveness in clinical settings. 


\section{Introduction}

Periods of transient fetal hypoxia are common during labor. Cardiotocography (CTG) is used to identify fetuses in hypoxic distress. The introduction of continuous CTG monitoring in the clinic led to an increase in caesarean sections. While the risk for developing seizures was reduced, neither neonatal mortality nor incidence of cerebral palsy did significantly decrease ${ }^{31}$. Therefore, additional markers for monitoring fetal distress have been considered. The ST interval of the fetal ECG represents the energyconsuming process of myocardial repolarization. The myocardium is unable to replenish its glycogen stores under hypoxic and acidotic conditions, leading to a change in the ST waveform ${ }^{34,67}$. Because a rise in the ST waveform of the fetal ECG was found to be associated with hypoxic distress ${ }^{32,33,68}$, an ST waveform analysis (STAN) device was introduced in many clinics as an addition to CTG. Subsequent meta-analyses of clinical trials, which evaluated the effect of STAN in addition to CTG, could not show a conclusive benefit of STAN on primary outcomes ${ }^{35,36}$. Despite that hypoxia and acidosis induce clear changes in the ST waveform, the lack of clinical benefit of STAN may be caused by the inability of the STAN device to detect changes in the ST waveform that indicate hypoxia and acidosis.

Therefore, we hypothesize that STAN is unable to discriminate between normoxic and hypoxic conditions by assessing the performance of the STAN device in a standardized ovine model with hypoxia-ischemia. In this standardized model fetal hypoxia-ischemia was induced by a single transient umbilical cord occlusion (UCO) of 25 minutes. ECG recordings were made using chronically implanted electrodes, and stored offline. Subsequently, ST waveform analysis was performed on ECG recordings fed into a STAN device. Resulting alarms were analyzed to evaluate the ability of STAN to discriminate between normoxic and hypoxic conditions.

\section{Methods}

\section{Animal experiment}

Animals The study group consisted of 8 fetuses of both genders from time-mated Texel ewes, randomly drawn from a larger cohort of 69 fetal sheep, which was measured over the period February 2010 to November $2013^{29,69,70}$. The original cohort studies - set up to investigate treatment of preterm hypoxic-ischemic encephalopathywere conducted at the Maastricht University Medical Center, Maastricht, the Netherlands, and were approved by the local Animal Ethics Research Committee (DEC 2010-131, DEC 2012-064). Use of this data for the current study affected neither measurement protocol nor study setup of the original studies.

Experiment Fetuses were instrumented at $101.7 \pm 1.0$ (mean \pm sd) days gestational age (GA; full gestation equals 146 days). Instrumentation was performed according to the procedure described previously by Jellema et al. ${ }^{29}$. During instrumentation 3 ECG electrodes ( $5 \mathrm{~mm}$; Cooner Wire Co., Chatsworth, $\mathrm{CA}$ ) were fixed subcutaneously with sutures to form ECG leads I, II and III, see Figure 1.2. An inflatable occluder (OC16HD, In Vivo Metric, Healdsburg, CA) was placed around the umbilical cord. A 3.5 French polyurethane umbilical vessel catheter (Tyco Healthcare Group, Mansfield, MA) was 
inserted into the femoral artery of the fetus to allow withdrawal of blood samples for blood gas analysis and for monitoring arterial blood pressure. Ewe and fetus were allowed to recover for 3 to 4 days. Subsequently, the umbilical occluder was inflated for 25 minutes to induce transient severe global hypoxia-ischemia.

\section{Data acquisition}

Blood gas analysis Fetal arterial blood was sampled to estimate arterial blood acidity level $(\mathrm{pH})$, arterial partial oxygen pressure $\left(\mathrm{PaO}_{2}\right)$ and arterial partial carbon dioxide pressure $\left(\mathrm{PaCO}_{2}\right)$. Baseline samples were drawn 1 hour before UCO. During UCO, samples were drawn at 5, 10 and 20 minutes after start of the UCO. In the reperfusion period, samples were drawn at 5, 10, 20, and 30 minutes after occlusion end.

Fetal scalp ECG ECG data were acquired and digitized by an MPAQ unit (MaastrichtProgrammable AcQuisition system, Maastricht Instruments BV, Maastricht, The Netherlands) at a sample frequency of $1000 \mathrm{~Hz}$. Matlab 2013a (The MathWorks, Natick, MA, USA) was used for further processing and analysis of ECG recordings from leads I and II during the period from 4 hours before umbilical cord occlusion until 4 hours after occlusion. Leads I and II were digitally projected to a negative aVF (-aVF) lead to reproduce the signal that would be measured using a unipolar scalp electrode. Subsequently, recordings were converted to an analog signal using the digital-analog converter on a Realtek ALC892 chipset (Realtek Semiconductor Corp., Taiwan). The analog signal was offered real time to a Goldtrace fetal scalp electrode (FSE-8000G, Neoventa Medical AB, Mölndal, Sweden) connected to a STAN S31 fetal heart monitor (Neoventa Medical AB). The heart rate monitor provided real time display of heart rate and ST waveform analysis. ECG displayed by the heart rate monitor resembled clinical fetal ECG in shape and amplitude. Screen output was simultaneously printed to paper by STAN P11 printer (Neoventa Medical AB). Alarms and other log entries were printed after the end of the recording. Logs were entered manually into Microsoft Excel version 14.0.7151.5001 (Microsoft Corporation, Redmond, WA) for further analysis with Matlab.

\section{Data analysis}

Based on clinical guidelines to STAN ${ }^{34}$, the following ST waveform-based alarms were considered significant: a biphasic ST waveform alarm lasting more than 2 minutes, a baseline T/QRS rise alarm over 0.05 and an episodic T/QRS rise alarm over 0.10 .

Performance analysis was based on alarms given by the STAN algorithm within 1 minute windows, which equals the temporal resolution of the log.

We used two approaches to analyze performance of the STAN device. To determine the time until the first alarm, we constructed a detection curve using the alarms during the UCO period. We did so for each of the three types of alarm separately as well as combined.

Using alarms generated during the 4 hour normoxic baseline period and during UCO, we determined sensitivity, specificity, positive predictive value (PPV), negative predictive value (NPV). We also determined Matthews correlation coefficient (MCC) as a measure of the correlation between the hypoxic/normoxic condition detected by STAN and the actual condition. A true positive result was defined as an alarm given within any 
1-minute window during the hypoxic UCO period. Consequently, a true negative result was defined as a 1-minute window without an alarm during the normoxic baseline period.

We furthermore analysed signal loss as reported through the log entries. The STAN device distinguishes two types of signal loss, namely loss of ST waveforms and loss of fetal heart rate. Loss of ST waveforms signifies intervals where the ST waveform could not be assessed by the device, but where HR could be assessed. HR loss indicates intervals where heart rate could not be determined by the device. We cross-referenced HR loss alarms with the input ECG to further specify causes of HR loss in the device. Three conditions were checked. The first was absent ECG signal due to subject handling, such as reconnecting the ECG leads to the MPAQ. The second and third conditions were high (>230 bpm) or low heart rates $(<50 \mathrm{bpm})$, above and below which the STAN device cannot determine HR.

\section{Results}

Blood gas measurements confirmed a successful umbilical cord occlusion. Partial arterial oxygen pressure decreased from 17 to $<5 \mathrm{mmHg}$, partial arterial carbon dioxide pressure increased from 39 to $109 \mathrm{mmHg}$ and arterial $\mathrm{pH}$ decreased from 7.42 to 6.88 at the end of UCO (Figure 2.1). Acidosis became more severe as hypoxia prolonged, with a concomitant rise in $\mathrm{PaCO}_{2}$. Release of UCO was followed by an immediate increase in $\mathrm{PaO}_{2}$ and gradual increases in arterial $\mathrm{pH}$ and $\mathrm{PaCO}_{2}$.

STAN produced few alarms during UCO. By the end of the UCO, when the fetus is severely asphyxiated and acidosis is present, baseline T/QRS rise alarms were given in three out of eight fetuses, episodic T/QRS alarms in four out of eight fetuses and biphasic ST alarms in three out of eight fetuses (Figure 2.2). For two of the fetuses, STAN did not produce any alarm.

Alarms were mostly absent during the UCO period, and generally abundant during the normoxic baseline period, as is shown in Figure 2.3. The absence of alarms during the hypoxic UCO period is reflected in the sensitivity of the different alarms (Table 2.1). Mean sensitivity for baseline T/QRS rise alarms, episodic T/QRS rise and biphasic ST alarms was $9 \%, 11 \%$ and $5 \%$, respectively. Despite the fact that blood gas measurements suggested normal blood acidity and normal arterial oxygen content during the baseline period, the STAN device produced many alarms in this period. The prevalence of false alarms during normoxia is indicated by low positive predictive values, which reflects what fraction of 1-minute windows contains STAN alarms corresponding to hypoxic conditions. PPV was $26 \%$ for baseline T/QRS rise, $32 \%$ for episodic T/QRS rise and 7\% for biphasic ST alarms. Low Matthews correlation coefficients for STAN alarms gave the same indication. Baseline T/QRS rise, episodic T/QRS rise and biphasic alarms were not, or weakly, correlated with normoxia/hypoxia ${ }^{71}$, with MCC of $0.12,0.07$ and -0.28 respectively. 
Low alarm sensitivity can be partially attributed to the fact that the STAN device has difficulties to assess the ST waveform during UCO (Figure 2.4 and Table 2.2). The STAN device could not assess the ST waveform in $58 \%$ of the 25 minutes of recording during UCO, either due to loss of ST waveform (48\%) or due to loss of heart rate (10\%). ST waveform loss was significantly increased during UCO with regards to the baseline $(17 \%$ to $48 \%$; Wilcoxon signed rank test: $p<0.05$ ).

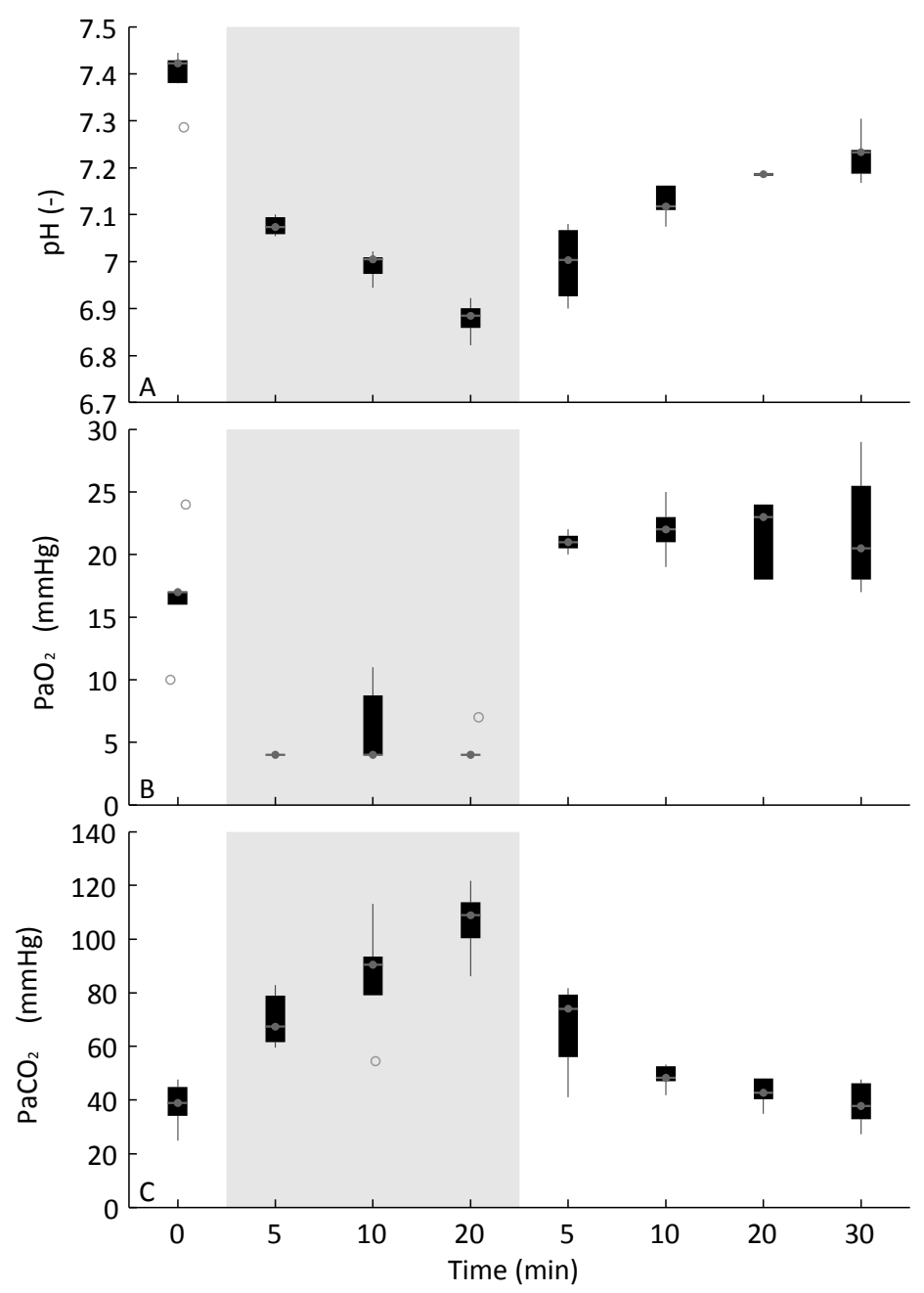

Figure 2.1: Whisker plots for arterial blood $\mathrm{pH}(\boldsymbol{A})$, partial arterial oxygen pressure (B) and partial arterial carbon dioxide pressure (C), with whiskers extending up to 1.5 times interquartile range. Blood gasses were assessed before, during (grey) and after umbilical cord occlusion. Outliers are displayed as open circles. 


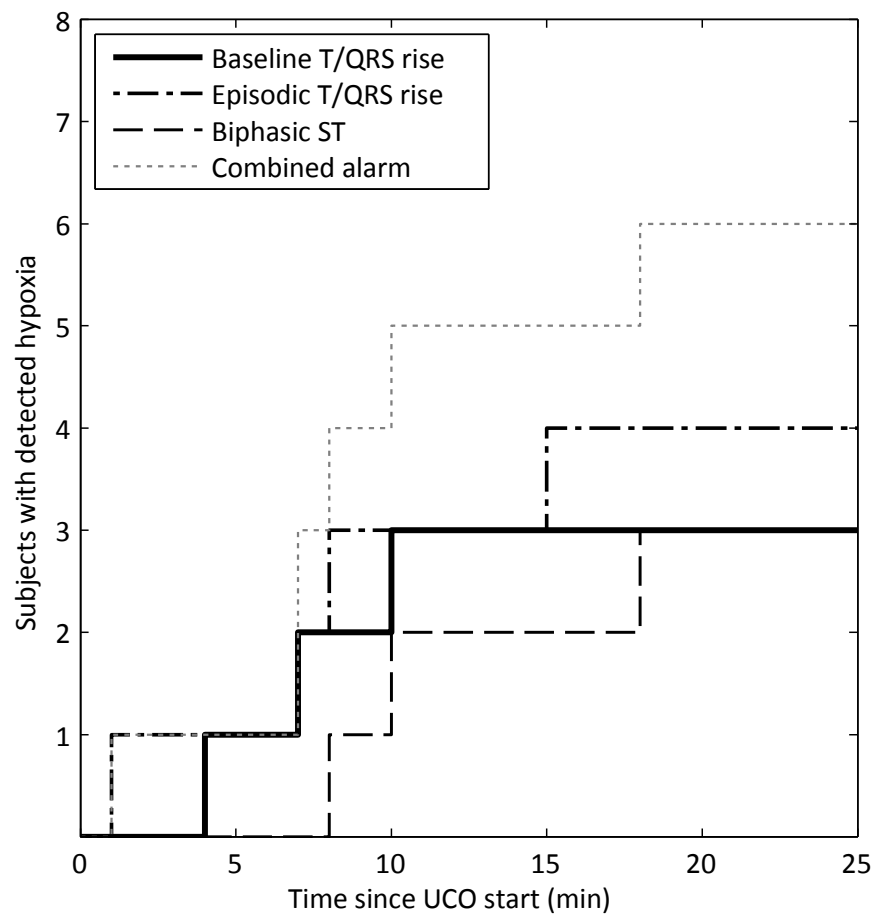

Figure 2.2: Detection of hypoxia by STAN alarms over the course of the umbilical cord occlusion (UCO).

Baseline rise Episodic rise Biphasic ST Combined alarm

\begin{tabular}{lrrrrrrrrrr}
\hline Sensitivity (\%) & 9 & $(0-35)$ & 11 & $(0-38)$ & 5 & $(0-19)$ & 20 & $(0-54)$ \\
Specificity (\%) & 96 & $(88-100)$ & 88 & $(69-100)$ & 58 & $(6-100)$ & 47 & $(6-89)$ \\
PPV (\%) & 26 & $(0-100)$ & 32 & $(0-100)$ & 7 & $(0-40)$ & 3 & $(0-$ & $0-9)$ \\
NPV (\%) & 91 & $(89-96)$ & 91 & $(87-94)$ & 79 & $(37-93)$ & 79 & $(34-93)$ \\
MCC (-) & $(-0.12-0.57)$ & $(-0.20-0.57)$ & $(-0.77-0.14)$ & $(-0.77-0.09)$
\end{tabular}

Table 2.1: Classification performance within subjects, based on 1 minute epochs during normoxia and UCO. PPV: positive predictive value; NPV: negative predictive value; MCC: Matthews correlation coefficient. Performance statistics are given as population mean (minimum subject value - maximum subject value). 

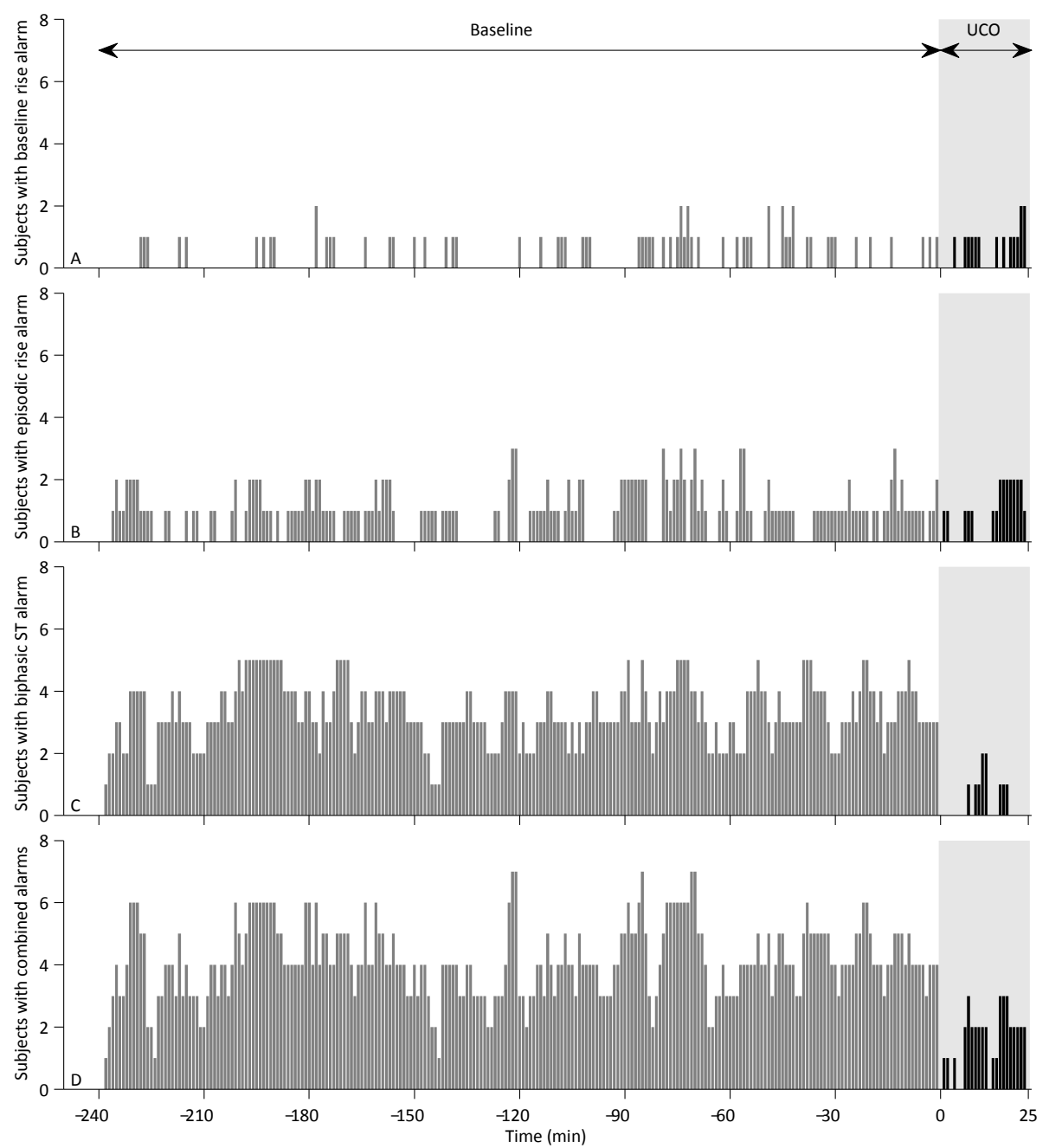

Figure 2.3: Number of fetuses with STAN alarms in 1 minute windows during the normoxic baseline period (grey bars on white background) and during the transient umbilical cord occlusion (black bars on grey background). The alarms assessed are baseline T/QRS rise $(\boldsymbol{A})$, episodic T/QRS rise (B) and biphasic ST alarms (C). When combining alarms (D) any alarm is considered, but only one alarm is counted each minute. During UCO, the STAN device suffered from high signal loss, which affects alarms. Notably, the STAN device could often not assess the ST waveform. 


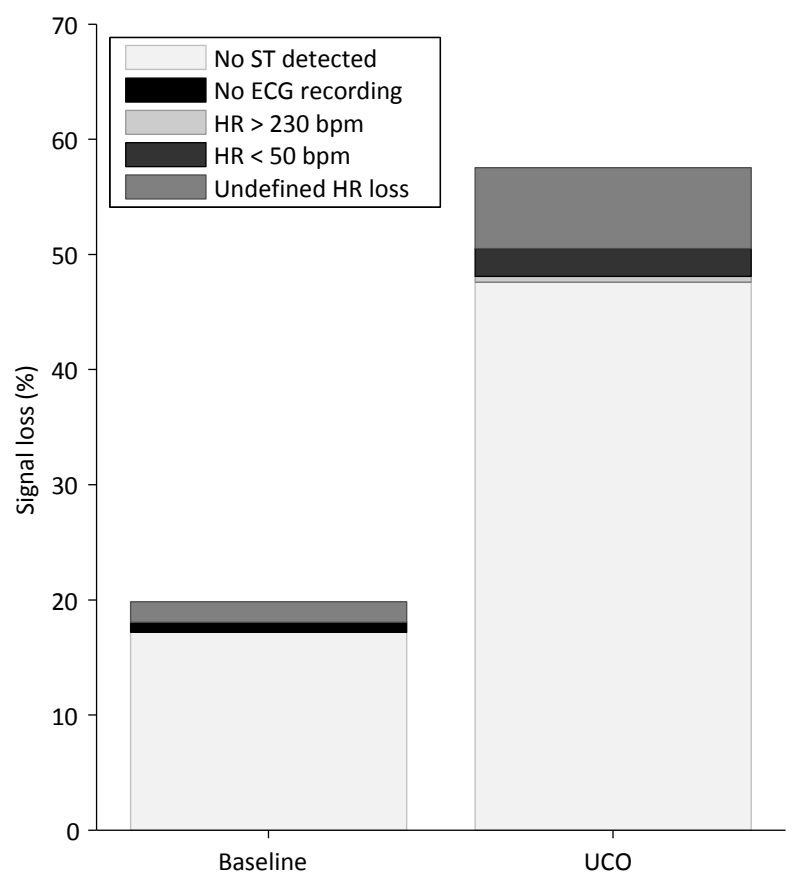

Figure 2.4: Causes of STAN device signal loss in the baseline period and during umbilical cord occlusion (UCO). In instances where no ST waveform could be assessed (No ST detected), heart rate (HR) could still be assessed. Absence of heart rate signal is caused by lack of ECG recording (No ECG recording), tachycardia (HR $>230 \mathrm{bpm})$, bradycardia ( $\mathrm{HR}<50 \mathrm{bpm}$ ) and undefined causes (Undefined HR loss). The latter is due to limitations of the STAN device itself, as ECG was present. 


\begin{tabular}{lrcrc} 
& Baseline & UCO \\
\hline No ST detected (\%) & $17(0-70) *$ & $48(15-88)$ \\
No HR detected (\%) & $3(0-10)$ & $10(0-50)$ \\
$\quad$ No ECG recording (\%) & $1(0-2) *$ & $0(0-0)$ \\
HR < 50 bpm (\%) & $0(0-0)$ & $2(0-19)$ \\
HR > 230 bpm (\%) & $0(0-0)$ & $0(0-4)$ \\
$\quad$ Undefined HR loss (\%) & $2(0-10)$ & $7(0-29)$ \\
Total signal loss (\%) & $20(3-70) \dagger$ & $58(15-100)$
\end{tabular}

Table 2.2: Signal loss in STAN recordings. STAN logs indicate segments where either ST waveforms cannot be assessed (No ST detected) or fetal heart rate is lost (No HR detected). Cross-reference of the latter with input ECG signal enabled specifying three causes of HR loss, i.e. absent recording (No ECG recording), severe bradycardia (HR $<50$ bpm) and severe tachycardia $(H R>230)$. The remaining HR loss indicated by STAN could not be attributed to these causes (Undefined HR loss) and occurred despite presence of ECG input signal. Values are population mean (minimum subject value - maximum subject value). Differences between baseline and UCO were assessed using the Wilcoxon signed rank test. *: $p<0.05 ;+: p<0.01$

\section{Discussion}

The STAN device was unable to adequately detect severe hypoxic fetal conditions in our standardized fetal ovine model for severe hypoxia-ischemia. A high presence of false alarms was indicated by a low positive predictive value of STAN alarms. Low alarm sensitivity demonstrated that there were but few alarms during UCO.

The use of an ovine model for fetal hypoxia-ischemia allowed evaluation of the STAN device within an environment where a clear distinction between normoxic and hypoxic state can be made. STAN guidelines indicate that a baseline period prior to the hypoxic event is required for the algorithm to properly function. Because short baseline periods could degrade detector performance ${ }^{34}$, we chose a baseline period of 4 hours to allow the STAN device to determine baseline T/QRS. After the baseline period followed 25 minutes of UCO during which severe hypoxia and acidosis developed. We expected that the severity and length of the hypoxic insult would allow the STAN device to detect the hypoxic condition of the fetus. Remarkably, alarms were mostly absent during the UCO period, and generally abundant during the normoxic baseline period.

The transient severe hypoxic-ischemic model is uncommon in clinical practice where hypoxia often develops more gradually. More gradual development of hypoxia is hypothesized to produce slower changes in ST waveform, as aerobic glycolysis could be maintained longer. Despite the different course of hypoxia in this study, we wonder whether in clinical practice alarms are produced correctly and timely and whether alarm fatigue might not be detrimental to correct assessmen ${ }^{72}$. The UCO period is long enough to allow both episodic and baseline T/QRS alarms to produce alarms. This does 
not happen consistently, and during normoxia alarms are produced as well. This could partially explain the findings from recent meta-analyses of clinic trials that demonstrated no significant benefit of the combination of CTG and STAN over CTG alone with regards to all primary outcomes except for the occurrence of seizures ${ }^{35,36}$.

We focused on STAN for detecting hypoxia. In clinical practice STAN supplements CTG surveillance. The abnormal heart rate tracing during UCO indicates fetal distress by itself, even without STAN. STAN was intended to provide additional information concerning fetal distress that could not be captured by CTG alone. In this study, where a period of definite distress was present, STAN produced a number of false alarms during normoxia and but few alarms during UCO, indicating that no or little additional information was provided.

We deliberately chose a fetal ovine model for evaluating performance of the STAN device. Not only was a fetal ovine model originally used to develop the STAN device ${ }^{33,34}$, it is furthermore regarded as a suitable animal model for studying fetal cardiovascular ${ }^{25}$ and cerebral ${ }^{26,30}$ responses to hypoxia-ischemia. Though clinical guidelines for STAN are for term infants, preterm and term fetuses demonstrate comparable hypoxia-induced ST waveform changes ${ }^{73,74}$. We therefore expect that STAN alarms should be comparable between preterm and term fetuses.

ECG leads I and II were not placed perfectly according to the Einthoven frame of reference. Therefore the reconstructed-aVF lead contained signal which is slightly different from signal measured using a scalp electrode. However, our signal was likely of better quality than that of a clinically measured unipolar scalp electrode for the following reasons. Any ECG waveform depends on the orientation between the recording electrodes and the electrical heart vector. The ST waveform is thus affected by how signal is measured. The electrical field produced by electrical cardiac activation and repolarization is propagated through tissue and liquids, including that of the mother. During birth the infant's head position, and thus the orientation of the scalp electrode with respect to the body, will vary. Since the electrical field produced by the fetal heart is propagated through maternal tissue, orientation of the scalp electrode with respect to the fetal heart vector will shift during birth. Thus the ST waveform is affected in clinical measurements, leading to increased variability. This is not the case in the -aVF lead which was reconstructed from two bipolar leads on the fetal body, and which thus provides a more reliable signal.

Identifying causes for the poor performance of STAN is impeded by the lack of description in literature concerning the specifics of its operation and implementation. One possible cause for poor performance of the STAN device lies in the hypoxia-induced changes in ST waveform. In several subjects within our study we observed that T waves became temporarily more negative during hypoxia, which was also described by others ${ }^{34,73}$. We doubt that the algorithm for STAN takes this possibility into account, as the name of baseline and episodic T/QRS rise alarms suggests that only positive T/QRS changes are assessed. If this is indeed the case, it would negatively affect STAN performance. 


\section{Conclusion}

A clinical STAN device could not detect hypoxia-ischemia after complete UCO in a standardized model for severe hypoxia-ischemia. Poor performance of the STAN

devicein assessing the normoxic/hypoxic state of a fetus with severe hypoxia-ischemia could indicate that in clinical settings the STAN device offers limited additional information concerning fetal hypoxic distress. 



\section{ECG markers for detecting hypoxia}

Submitted:

Zwanenburg, A., Hermans, B. J. M.,Andriessen, P., Niemarkt, H. J., Jellema, R. K., Ophelders, D. R. M. G., Vullings, R., Wolfs, T. G. A. M., Kramer, B. W. \& Delhaas, T. A comparison of ECG-based physiological markers for hypoxia in a preterm ovine model. Ped Res (2015) 



\begin{abstract}
Background Current methods for assessing perinatal hypoxic conditions did not improve infant outcomes. Various waveform-based and interval-based ECG markers have been suggested, but not directly compared. We compare performance of ECG markers in a standardised ovine model for fetal hypoxia.
\end{abstract}

Methods 69 fetal sheep of 0.7 of gestation had ECG recorded 4 hours before, during and 4 hours after a 25 minute period of transient umbilical cord occlusion (UCO), leading to severe hypoxia. Interval- and waveform-based ECG marker values were calculated. Performance was assessed by correlating predicted and actual hypoxic/normoxic state and subsequently deriving sensitivity, specificity and positive predictive value (PPV), and determining the accumulative detection rate of hypoxia during UCO.

Results Of the markers studied, HR, QTC Bazett $_{\text {and }}$ QTC Hodges $_{\text {demonstrated high }}$ sensitivity ( $\geq 86 \%)$, specificity ( $\geq 96 \%)$ and PPV ( $\geq 86 \%)$. These marker detected hypoxia in $\geq 90 \%$ of fetuses at 4 minutes after UCO. In contrast, episodic and baseline T/QRS rise markers, analogue to alarms of clinical ST waveform analysis devices, displayed low sensitivity ( $\leq 20 \%)$ and could not detect severe fetal hypoxia in $65 \%$ and $28 \%$ of the animals respectively.

Conclusion Interval-based heart rate, $\mathrm{QTC}_{\mathrm{Bazett}}$ and $\mathrm{QTC} \mathrm{C}_{\text {Hodges }}$ markers could assess the presence of severe hypoxia. Waveform-based episodic and baseline T/QRS rise markers were ineffective as markers for hypoxia. 


\section{Introduction}

Continuous monitoring of the fetal heart rate is assumed to provide clinical information about the well-being of the fetus ${ }^{31}$. Cardiotocography (CTG) allows noninvasive real-time assessment of fetal heart rate and its response to uterine contractions, and is nowadays used in clinics worldwide. However, heart rate patterns in CTG are often not predictive for hypoxia ${ }^{75}$. Introduction of CTG in clinics did not significantly reducing occurrences of neonatal death or cerebral palsy, but did lead to an increase in caesarean sections ${ }^{31}$. Consequently, ST waveform analysis (STAN) was proposed as an additional analysis method. STAN assesses hypoxia-induced changes in the ST waveform of the $\mathrm{ECG}^{76}$ and operates in conjunction with CTG. Implementation of STAN in clinical practice was initially associated with a beneficial reduction in hypoxiainduced outcomes (metabolic acidosis and moderate/ severe neonatal encephalopathy) compared to CTG alone ${ }^{68}$. Recent meta-analyses of clinical trials, however, demonstrated no clear advantage of STAN with CTG over CTG alone with respect to primary outcomes ${ }^{35,36}$.

STAN markers are based on the shape of the ECG waveform. Other ECG markers for hypoxia detection have been suggested, namely atrioventricular conduction delay ${ }^{37,38}$, the relationship of AV delay and heart rate $\mathrm{e}^{39,40}$ and ventricular activation and repolarisation ${ }^{41}$. These markers are all interval-based, i.e. they use time intervals between characteristic points in the ECG. A major difference between waveform-based markers and interval-based markers is the dependency of the former on the projection angle of the ECG lead with the heart vector. Variation in orientation of the heart vector can lead to different results for waveform-based markers. An example, based on T/QRS ratio, is demonstrated in Figure 3.1. Interval-based markers are less dependent on differences in projection angle and might perform better in detecting fetal hypoxia.

Direct comparison of ECG markers is hampered because the different markers have previously been studied under different circumstances. Therefore, we compare performance of waveform- and interval-based ECG markers for hypoxia in a standardized pre-clinical ovine fetal model of hypoxia-ischemia.

\section{Methods}

\section{Animal experiment}

Animals The study group for this retrospective study consists of 74 fetuses of both genders of time-mated Texel ewes, measured in the period from February 2010 to November 2013. The study group was originally set up to investigate processes and treatment of preterm hypoxic-ischemic encephalopathy ${ }^{29,69,70}$. The original studies were approved by the local Animal Ethics Research Committee (approval reference numbers: DEC 2010-131, DEC 2012-064) and conducted at the Maastricht University Medical Center, Maastricht, the Netherlands. The current study did not require additional approval. 

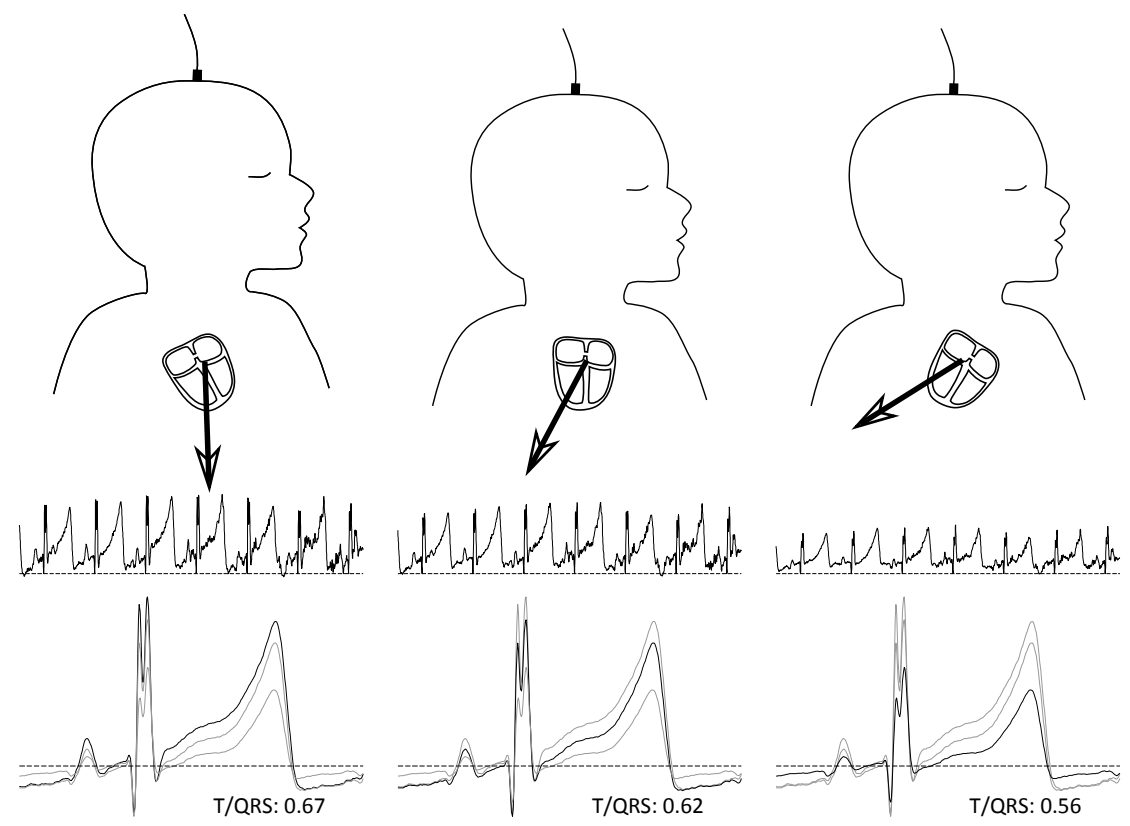

Figure 3.1: Influence of heart axis orientation on waveform-based T/QRS values. The same signal is projected to simulate a rotation of the heart axis by 30 and 60 degrees to the right. This affects the shape of the ECG waveform and the T/QRS ratio, defined as the maximum deviation of the $T$ wave with regards to baseline divided by the amplitude of the QRS complex.

Instrumentation Fetuses were instrumented at $101.5 \pm 1.2$ (mean \pm sd) days gestational age (GA; full gestation equals 146 days). The surgical procedure was described previously ${ }^{29}$. Ewes received intravenous prophylactic antibiotics (1000 mg amoxicillin and $200 \mathrm{mg}$ clavulanic acid). Anesthesia was induced by intravenously administered thiopenthal ( $15 \mathrm{mg} / \mathrm{kg}$ ). After intubation, general anesthesia was maintained with 1 to $2 \%$ isoflurane guided by depth of sedation and supplemented by remifentanyl i.v. (0.75 $\mu \mathrm{g} / \mathrm{kg} / \mathrm{min}$ ) for analgesia. During surgery, vital parameters and depth of sedation were continuously monitored by certified personnel. A catheter was placed in the maternal long saphenous vein to provide access for a peri-operative saline drip ( $250 \mathrm{~mL} / \mathrm{hour}$ ) and for postoperative blood sampling and administration of prophylactic antibiotics for four days.

The fetus was exposed through a lower midline laparotomy. Two catheters (3.5 French polyurethane umbilical vessel catheters; Tyco Healthcare Group, Mansfield, MA) were inserted in the brachial vein and femoral artery. A vascular occluder (OC16HD, In Vivo Metric, Healdsburg, CA) was placed around the umbilical cord. Three silver electrodes (5mm; Cooner Wire Co., Chatsworth, CA) were fixed subcutaneously with sutures for continuous fetal ECG recording, forming ECG leads I, II and III. After repositioning the fetus, an amniotic catheter was inserted into the amniotic cavity and 
used to fill the cavity with warm sterile saline and $80 \mathrm{mg}$ of Gentamycin. The electrode leads and catheters were exteriorised through the flank of the ewe and incisions were sutured. After surgery, catheters were continuously perfused with heparinized saline $(25 \mathrm{IU} / \mathrm{mL}, 0.2 \mathrm{~mL} / \mathrm{h})$. The ewe and fetus were allowed to recover from surgery for three to four days. At $105.6 \pm 1.0$ (mean \pm sd) days, the umbilical cord was occluded for 25 minutes by rapidly inflating the occluder with sterile saline. Blood pressure and heart rate were monitored and intermittent blood gas measurements were performed before, during and after UCO.

Animals were housed together when possible and had ad libitum access to water and food. Surgical wounds were inspected daily and treated with chlortetracycline spray to prevent infection. The welfare of the animals was monitored daily by certified personnel. The animals were maintained for 7 days after occlusion. At this point both ewe and fetus were euthanized by intravenous administration of pentobarbital (200 $\mathrm{mg} / \mathrm{kg})$.

\section{Data acquisition}

ECG data were acquired and digitized by an MPAQ unit (Maastricht-Programmable AcQuisition system, Maastricht Instruments BV, Maastricht, The Netherlands) (26) at a sample frequency of $250 \mathrm{~Hz}$ (12 fetuses) or $1000 \mathrm{~Hz}$ ( 57 fetuses). After measurement in the initial cohort of 12 fetuses, it was decided to increase the sample frequency from $250 \mathrm{~Hz}$ to $1000 \mathrm{~Hz}$ to capture ECG features at a higher temporal resolution. ECG data were analysed from 4 hours before umbilical cord occlusion until 4 hours after occlusion.

\section{Data processing}

All data processing and analysis was automatically performed using custom scripts in Matlab 2013b (The MathWorks, Natick, MA, USA). The first step of the analysis was preprocessing ECG data for each fetus to derive averaged ECG waveforms ( $E_{\text {CG }}$ avg) for every 30 beats. The pre-processing steps are illustrated in figure 4. In short, ECG was filtered using a $0.05-100 \mathrm{~Hz}$ third-order bidirectional Butterworth bandpass filter and a $50 \mathrm{~Hz}$ third-order bidirectional infinite impulse response notch filter to reduce signal drift, high frequency noise and mains noise (Figure 3.2A). To remove major baseline deviations, the ECG was subsequently decomposed using a Daubechies 5-wavelet, up to the 6 th $(250 \mathrm{~Hz})$ or 8 th $(1000 \mathrm{~Hz})$ level ${ }^{77}$. The residual was regarded as a baseline deviation and subtracted from the ECG signal (Figure 3.2B). To imitate the unipolar scalp electrode used to record invasive ECG for ST waveform analyses, leads I and II were digitally projected to a negative aVF lead. To determine cycle length $(C L)$, and to define a suitable feature for waveform averaging, we determined the position of the $Q$ wave top in the -aVF lead using our adaptation of the Pan Tompkins algorithm (Figure $3.2 \mathrm{C})^{78}$. Q wave top was used instead of $R$ wave top because the $R$ wave was split in all fetuses, the split $R$ wave tops varied in amplitude and position, and therefore did not represent a stable feature in the ECG. Differences between vertical positions of subsequent $Q$ wave tops were linearly interpolated and subtracted, thus aligning each $Q$ wave top with 0 (Figure 3.2D) ${ }^{79}$. To derive $\mathrm{ECG}_{\text {avg, }}$, we set the 25 th percentile of cycle lengths from 30 subsequent ECG waveforms ( $E C G_{\text {ind }}$ ) as CL25. Each of the $30 \mathrm{ECG}$ waveforms was truncated in the interval $[\mathrm{Q}-0.4 * \mathrm{CL} 25, \mathrm{Q}+0.8 * \mathrm{CL25}]$. This interval was chosen to 
A ) Filtered ECG $(0.05-100 \mathrm{~Hz})$

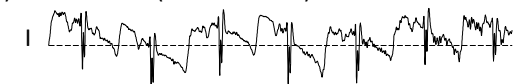

B ) Wavelet baseline correction

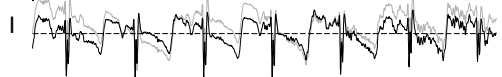

C) Q wave top detection

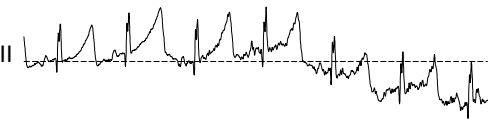

1

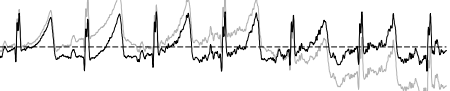

$-\mathrm{aVF}$

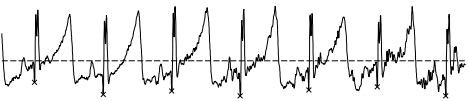

D ) Linear baseline correction

$-a V F$

E) Average ECG waveform
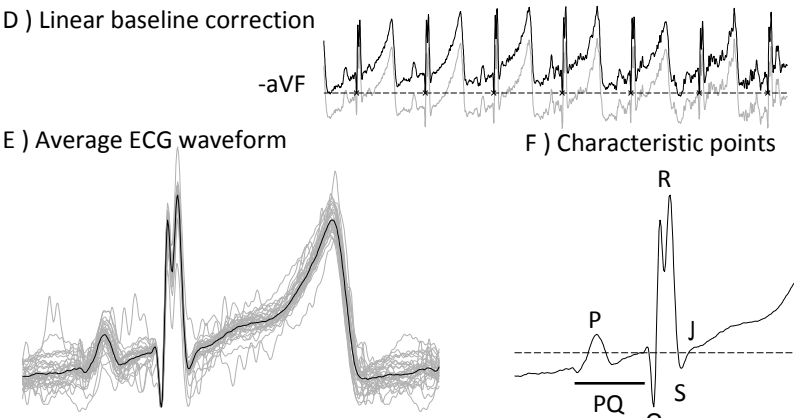

F) Characteristic points

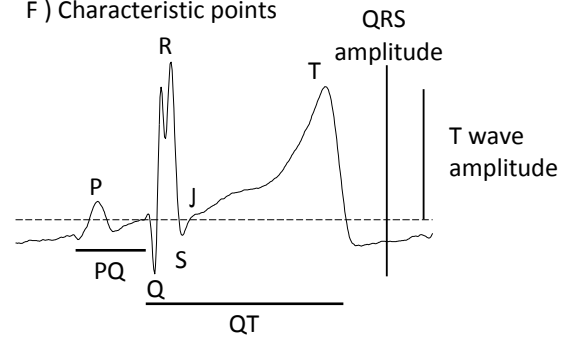

Figure 3.2: Illustration of ECG pre-processing steps. (A) ECG recordings for leads I and II are first filtered using a Butterworth filter. (B) Subsequently, the filtered signal (grey) is decomposed using a wavelet and partially reconstructed (black) to suppress baseline wander. (C) $Q$ wave tops (black cross) are detected after projecting ECG I and II leads to a negative aVF lead. (D) Amplitude differences between $Q$ wave tops are linearly interpolated and used for further correction of baseline wander of the ECG. (E) 30 truncated ECG waveforms are aligned on the detected $Q$ wave top, and averaged. (F) Characteristic points, intervals and waves of the ECG. T amplitude is the amplitude of the $T$ wave above baseline and QRS amplitude is the largest of $Q R$ and RS amplitudes.

capture relevant features and intervals of the ECG waveform. The truncated waveforms were averaged by omitting $30 \%$ of the outermost values at each sample point and calculating the mean over the remaining $70 \%$ (Figure 3.2E). Occasionally, $\mathrm{ECG}_{\text {avg }}$ thus derived should be omitted due to low signal quality of part of the $30 \mathrm{ECG}_{\text {ind }}$ from which $E C G_{\text {avg }}$ was derived. Quality of $\mathrm{ECG}_{\text {avg }}$ was verified by deriving a signal-to-noise ratio (SNR). This SNR was defined as the base-10 logarithm of the squared length of $\mathrm{ECG}_{\text {avg }}-\mathrm{a}$ surrogate for true signal variance-divided by the mean squared length of the corresponding $30 \mathrm{ECG}_{\text {ind }}$-a surrogate for noise induced variance. Waveforms with SNR below the empirically established threshold of -0.25 were removed from further analysis.

ECG-based physiological markers

Figure 3.2F illustrates characteristic points, segments and intervals of the ECG. Q wave onset, $P$ wave onset, $R$ wave top, $S$ wave top, $J$ point and $T$ wave end were detected in $\mathrm{ECG}_{\text {avg }}$ using our adaptation of the Pan 
Tompkins algorithm. $\mathrm{Q}$ wave onset and $\mathrm{P}$ wave onset were also detected in $\mathrm{ECG}_{\text {ind. }} \mathrm{Q}$ wave onset was used as ECG baseline, since at high heart rates the T wave partially overlaps and merges with the $P$ wave, thereby slanting the PQ segment commonly used to define the ECG baseline.

Values for all ECG markers were updated every 30 beats, i.e. at every subsequent derivation of $\mathrm{ECG}_{\mathrm{avg}}$. The values of the various markers were calculated as follows.

Heart rate was determined by calculation of the mean heart rate of the 30 most recent $E C G_{\text {ind }}{ }^{80}$. Heart rate variability was calculated by determining the standard deviation of heart rates of for the same 30 most recent $E C G_{\text {ind }}{ }^{80}$.

Atrioventricular conduction delay was estimated by the length of the PR interval. The PR interval was defined as the interval between the onset of the $P$ wave and the start of the QRS complex. The PR/RR ratio is calculated for by dividing the PR interval by the RR interval for the 30 most recent $\mathrm{ECG}_{\text {ind }}$ and calculating their mean value. We used the approach of Van Wijngaarden et al. to assess the PR-HR relationship, and calculated the Pearson correlation coefficient between PR intervals and heart rates of $E_{C G}$ ind in the previous 150 seconds $^{39}$.

QT interval length represents ventricular activation and repolarisation time. The QT interval was defined as the time interval between the onset of the $Q$ wave and the end of the T wave in ECGavg. QT interval length decreases with increasing heart rate and is therefore corrected for heart rate to reduce HR dependency. In addition to Hodges' correction, $Q T_{c, \text { Hodges }}=Q T+1.75(H R-60)$, and Bazett's correction. $Q T_{c, \text { Bazett }}=Q T(H R / 60)^{1 / 2}$, we also assessed the uncorrected $Q T$ interval.

T/QRS ratio was calculated as the maximal positive or negative deflection between $S$ wave top or J point-when present-and the end of the T wave, divided by the amplitude of the QRS complex in ECGavg (Figure 3.2F). The clinically used and commercially available STAN device assesses episodic and baseline rise in T/QRS values as ECG markers for hypoxia ${ }^{76}$. Analogue to STAN device alarms, episodic rise in T/QRS value was defined as a positive change between the actual T/QRS value and the median T/QRS value of the previous 10 minutes $^{68}$. Also analogue to STAN device alarms, baseline rise in T/QRS value was defined as a positive change from baseline by comparison of the median T/QRS value of the last 10 minutes with the baseline T/QRS value $^{68}$. The baseline T/QRS value was defined as the lowest non-negative median T/QRS value in a 20 minute moving window within the previous 3 hours of recording.

Blood gasses We sampled fetal arterial blood to obtain baseline values for blood acidity $(\mathrm{pH})$, arterial partial oxygen pressure $\left(\mathrm{PaO}_{2}\right)$ and arterial partial carbon dioxide pressure $\left(\mathrm{PaCO}_{2}\right)$. Samples for the baseline period were drawn 1 hour before UCO took place. During UCO samples were withdrawn at 5, 10, 15, and 20 minutes after UCO start. In the recovery period samples were drawn at 3,10, 20,30, and 60 minutes after UCO ended.

\section{Performance analysis}

Interval- and waveform-based ECG markers were grouped within 1-minute windows as follows to facilitate comparison between markers. Markers within each 1-minute 
window were represented by the median value, except for STAN episodic and baseline rise markers, which were represented by the maximum value within the 1-minute window. We based performance analysis on changes in marker values with respect to the individual baseline. We defined the individual baseline as the mean value of the marker during the baseline period for the individual under consideration. STAN episodic or baseline rise markers were not corrected for the individual baseline, as they were already corrected for baseline in a different way. Subsequently, the individual baseline value was subtracted from windowed marker values to determine (normalised) changes with respect to baseline.

Area under the receiver operating characteristic (ROC) curve (AUC) is a general measure for the ability of an ECG marker to differentiate between hypoxic and normoxic states. To construct ROC curves, normalised values during the baseline and UCO period were first sorted. Then detection thresholds for positive and negative changes were iteratively increased. A contingency table was calculated for each combination of positive and negative thresholds. Sensitivity and specificity were calculated from these contingency tables for the purpose of creating the ROC curve. Subsequently AUC was calculated.

Matthews correlation coefficient (MCC) ${ }^{81,82}$ was calculated for each of the contingency tables underlying the ROC curve to define the optimal correlation between actual and predicted hypoxic/normoxic states. The positive and negative thresholds corresponding to the optimal correlation were used to define sensitivity, specificity, positive (PPV) and negative predictive values (NPV) (Figure 3.3 and 3.4).

Performance analysis using ROC disregards the temporal behaviour of ECG markers during UCO. To assess the ability of ECG-based markers to distinguish between a normoxic and hypoxic state on the individual level, we constructed cumulative detection curves. The detection curves were subsequently used to tabulate the percentage of detected individuals at several time points during UCO. The percentage of detected individuals was assessed at 4 minutes (onset of metabolic acidemia; $\mathrm{pH}<$ $7.1^{83,84}$ ), 12 minutes (progressive acidemia; $\mathrm{pH}<7.0^{85}$ and at the end of the umbilical cord occlusion at 25 minutes. 


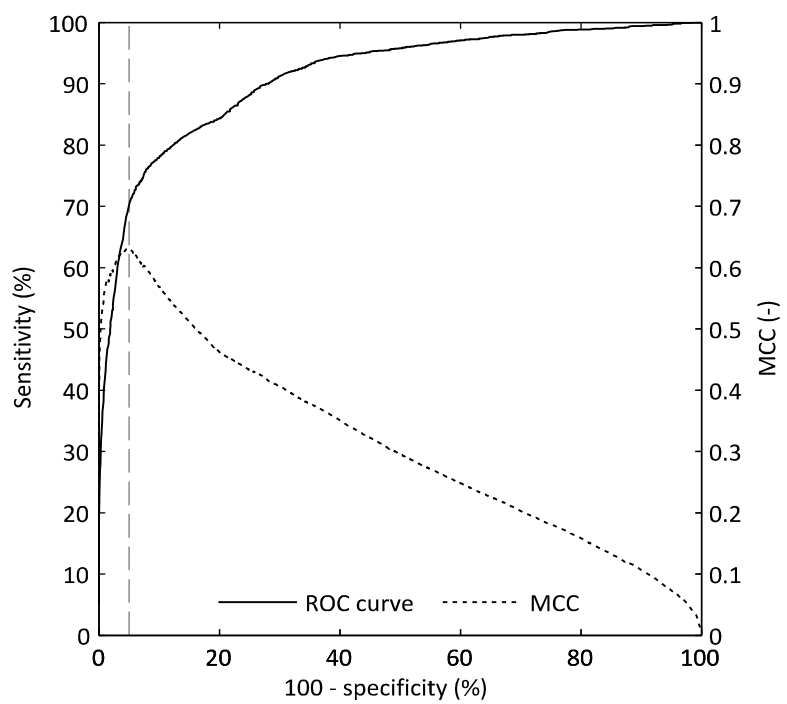

Figure 3.3: ROC curve for T/QRS ratio, with Matthews correlation coefficient (MCC) describing the correlation between predicted and actual hypoxic/normoxic state. The position of maximum MCC is shown on the ROC curve (vertical dashed line).

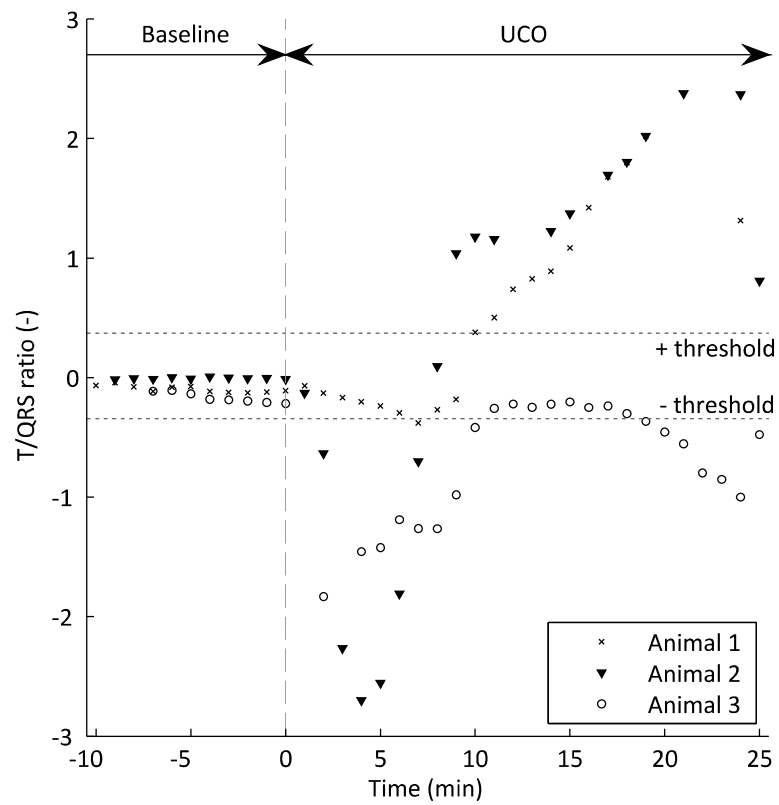

Figure 3.4: Thresholds for changes in T/QRS ratio with respect to individual baseline. T/QRS values of three subjects are shown. To clearly show T/QRS values during UCO only part of the baseline is shown. An alarm for hypoxia was given if the change in T/QRS ratio was above the positive threshold (+; T/QRS $=0.37$ ) or was below the negative $(-; T / Q R S=-0.34)$ threshold . 


\section{Results}

Presence of umbilical cord occlusion induced hypoxia-ischemia

Blood gas measurements confirm a clinically relevant umbilical cord occlusion (UCO), with a decrease in partial oxygen pressure, an increase in partial carbon dioxide pressure, $\mathrm{pH}<7.1$ (Figure 3.5), and bradycardia (Figure 3.6). Heart rate accelerates to above normal values within the first 15 minutes after resolution of the UCO. It then returns to almost normal values within the first hour after UCO and subsequently gradually increases again. Arterial $\mathrm{pH}$ values recover within the first hour. Arterial blood oxygen content directly rises after UCO, and remains elevated during the first hour after UCO, whereas carbon dioxide content decreases to normal values within 10 minutes.

\section{Classification performance of ECG markers}

Interval-based markers for hypoxia generally performed better than waveformbased markers. Heart rate, $\mathrm{PR} / \mathrm{RR}$ ratio, and heart rate corrected $\mathrm{QT}_{\mathrm{c}, \text { Bazett }}$ and $\mathrm{QT}_{\mathrm{c} \text {,Hodges }}$ markers demonstrate near-perfect behaviour on the receiver operating characteristic (ROC) curve (Figure 3.7), which is reflected in the area under curve (AUC) value (Table 3.1). In contrast, both STAN-device analogue episodic and baseline rise markers show AUC values close to random (0.50). This contrast is likewise visible in the degree of correlation between predicted and actual hypoxic/normoxic states. Interval-based HRV and waveform-based episodic and baseline T/QRS rise markers show a weak correlation (Matthews correlation coefficient; $\mathrm{MCC} \leq 0.35)^{71}$, whereas interval-based heart rate, $\mathrm{PR} / \mathrm{RR}$ ratio and both heart rate corrected QT markers were strongly correlated (MCC $>0.67$ ).

\section{Identification of hypoxic individuals during UCO}

Low sensitivity does not directly imply that on the individual level hypoxia will not be detected. We tested the individual detection rates based on the assumption that a single positive result during the UCO period would constitute a detection of hypoxia. Accumulative detection of individuals during UCO is shown in Figure 3.8. The percentage of individuals with marker values indicating hypoxia at the 4 minute mark, 12 minute mark and end of the UCO is shown in Table 3.2. At the 4 minute mark $(\mathrm{pH}<7.1)$, hypoxia can be most clearly distinguished by assessing heart rate $(96 \%$ of individuals), PR/RR ratio (96\%), and Hodges' (91\%) and Bazett's corrected QT length (90\%). By 12 minutes of hypoxia ( $\mathrm{pH}<7.0$ ), these markers identify $97 \%$ to $99 \%$ of the fetuses as hypoxic. Waveform-based STAN episodic and baseline rise markers for hypoxia do not perform well. $65 \%$ and $28 \%$ of the severely asphyxiated individuals were not detected by the end of the UCO using these markers.

\section{Baseline and threshold values}

An overview of population mean baseline values and positive and negative threshold values for predicting hypoxic/normoxic state is shown in Table 3.3. Alarms were produced when the change with respect to the baseline value exceeded the positive threshold, or decreased below the negative threshold. Heart rate, HRV and $\mathrm{PR} / \mathrm{RR}$ ratio markers produced alarms when values decreased below the negative 
threshold. The STAN-device analogue episodic and baseline rise markers only produced alarms when values exceeded the positive threshold. Other markers produced alarms above positive and below negative threshold values. Notably, the T/QRS marker produced $53 \%$ of its alarms when it exceeded the positive threshold and $47 \%$ of its alarms when it decreased below the negative threshold, in contrast to the STAN-device analogue markers that only produce alarms on positive changes. 


\begin{tabular}{lrrrrrr} 
ECG marker & Sensitivity & Specificity & PPV & NPV & MCC & AUC \\
\hline Heart rate & 0.96 & 1.00 & 0.98 & 0.99 & 0.97 & 0.99 \\
HRV & 0.58 & 0.85 & 0.34 & 0.94 & 0.34 & 0.79 \\
PR interval & 0.75 & 0.99 & 0.91 & 0.97 & 0.80 & 0.95 \\
PR/RR ratio & 0.90 & 0.99 & 0.93 & 0.99 & 0.91 & 0.97 \\
PR-HR correlation & 0.37 & 0.97 & 0.64 & 0.92 & 0.44 & 0.79 \\
QT $_{\text {uncorrected }}$ & 0.50 & 0.95 & 0.53 & 0.94 & 0.46 & 0.83 \\
QTC $_{\text {Bazett }}$ & 0.86 & 0.98 & 0.86 & 0.98 & 0.84 & 0.97 \\
QTC Hodges $_{\text {T/QRS ratio }}$ & 0.94 & 1.00 & 0.98 & 0.99 & 0.96 & 0.99 \\
STAN episodic rise $^{\mathrm{a}}$ & 0.70 & 0.95 & 0.65 & $\mathbf{0 . 9 6}$ & 0.63 & $\mathbf{0 . 9 1}$ \\
STAN baseline rise $^{\mathrm{a}}$ & 0.10 & $\mathbf{1 . 0 0}$ & 0.84 & 0.88 & 0.26 & 0.58 \\
\end{tabular}

Table 3.1: Classification performance of individual ECG markers for hypoxia. PPV: positive predictive value; NPV: negative predictive value; MCC: Matthews correlation coefficient; AUC: Area under the receiver-operator characteristic curve; Sensitivy, specificity, PPV and NPV were determined using the contingency table corresponding to maximum MCC. Performance values above 0.90 are emphasized using boldface.

${ }^{a}$ Marker is not corrected for individual baseline, by definition.

\begin{tabular}{lrrr} 
ECG marker & $4 \mathrm{~min}(\%)$ & $12 \mathrm{~min}(\%)$ & End (\%) \\
\hline Heart rate & 96 & 99 & 100 \\
HRV & 35 & 79 & 85 \\
PR interval & 75 & 93 & 96 \\
PR/RR ratio & 96 & 97 & 100 \\
PR-HR correlation & 50 & 79 & 84 \\
QT $_{\text {uncorrected }}$ & 78 & 91 & 94 \\
QTC $_{\text {Bazett }}$ & 90 & 97 & 100 \\
QTC $_{\text {Hodges }}$ & 91 & 97 & 100 \\
T/QRS ratio $_{\text {STAN episodic rise }}{ }^{\mathrm{a}}$ & 81 & 94 & 100 \\
STAN baseline rise $^{\mathrm{a}}$ & 1 & 13 & 35 \\
\end{tabular}

Table 3.2: Accumulative detection of hypoxic individuals, based on deviations from individual baselines in ECG marker values, at $4 \mathrm{~min}(\mathrm{pH}<7.1), 12 \mathrm{~min}(\mathrm{pH}<7.0)$ and the end of the umbilical cord occlusion. Detection rates above $90 \%$ are emphasized using boldface.

${ }^{a}$ Marker is not corrected for individual baseline, by definition. 


\begin{tabular}{|c|c|c|c|c|c|}
\hline \multirow[b]{2}{*}{ ECG marker } & \multirow[b]{2}{*}{ Baseline } & \multicolumn{2}{|c|}{ Threshold } & \multicolumn{2}{|c|}{ Distribution (\%) } \\
\hline & & + & - & + & - \\
\hline Heart rate (bpm) & $179(155-204)$ & NA & -37 & NA & 100 \\
\hline HRV (bpm) & $2.11 \quad(0.26-3.95)$ & NA & -1.12 & NA & 100 \\
\hline PR interval (ms) & $64 \quad(52-76)$ & 7 & -9 & 92 & 8 \\
\hline PR/RR ratio & $0.19(0.15-0.23)$ & NA & -0.03 & NA & 100 \\
\hline PR-HR correlation & $-0.07(-0.35-0.21)$ & 0.37 & -0.37 & 64 & 36 \\
\hline $\mathrm{QT}_{\text {uncorrected }}(\mathrm{ms})$ & $193(162-224)$ & 23 & -35 & 67 & 33 \\
\hline $\mathrm{QTC}_{\text {Bazett }}(\mathrm{ms})$ & $332(284-380)$ & 134 & -53 & 10 & 90 \\
\hline $\mathrm{QTC}_{\text {Hodges }}(\mathrm{ms})$ & $401 \quad(362-440)$ & 98 & -83 & 10 & 90 \\
\hline T/QRS ratio & $-0.20(-0.60-0.21)$ & 0.37 & -0.34 & 53 & 47 \\
\hline STAN episodic rise ${ }^{a}$ & $0.00(0.00-0.00)$ & 0.48 & NA & 100 & NA \\
\hline STAN baseline rise $\mathrm{e}^{\mathrm{a}}$ & $0.00 \quad(0.00-0.00)$ & 0.13 & NA & 100 & NA \\
\hline
\end{tabular}

Table 3.3: Population mean baseline value and the corresponding 95\% prediction interval were calculated from 69 individual baseline values. The threshold denotes a change with respect to the individual baseline. Thresholds were constructed through a data-driven approach by optimizing correlation between predicted and actual normoxic/hypoxic state. A change with respect to individual baseline produced a detection if the change was above the positive (+) threshold or below the negative (-) threshold. The distribution of detections during UCO is divided into two categories. The first category (+) is the fraction of total detections that was due to changes above the positive threshold. The second category (-) is the complement of the first, being the fraction of total detections that was due to changes below the negative threshold. NA: threshold did not lead to any detections during UCO.

${ }^{a}$ Marker is not corrected for individual baseline, by definition. 


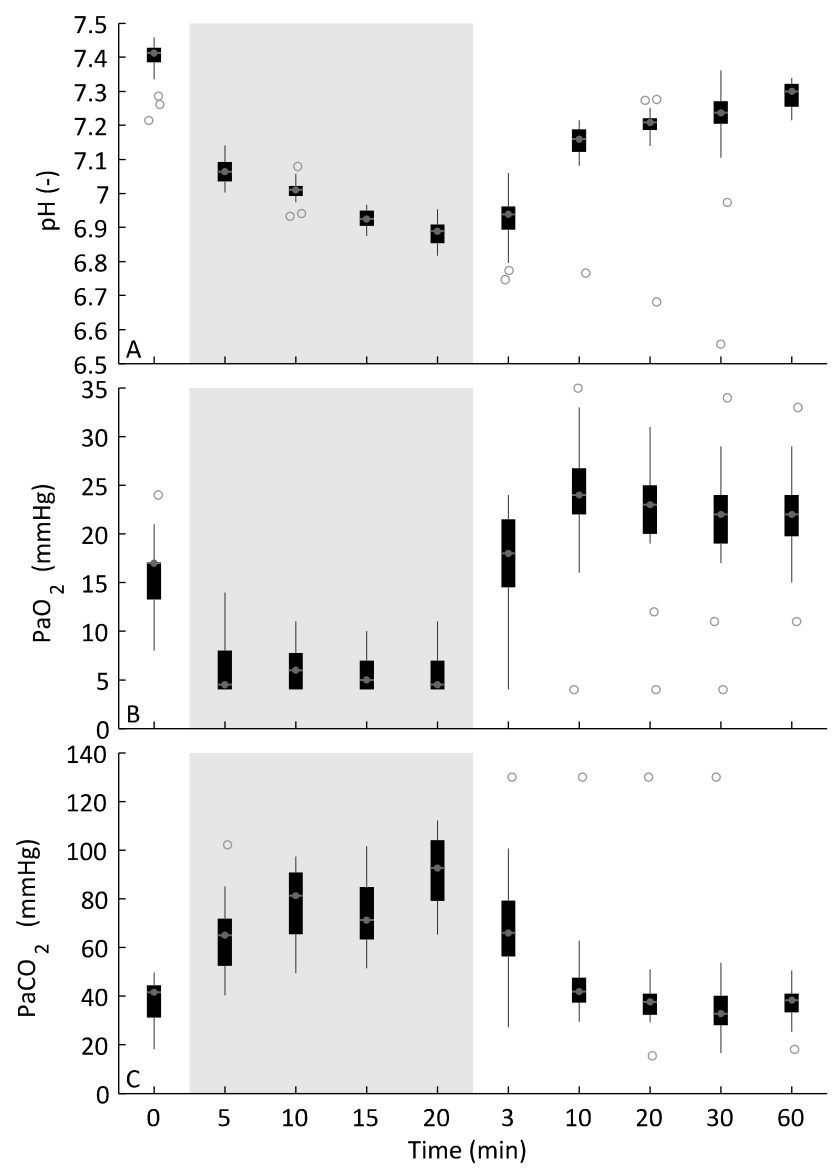

Figure 3.5: Whisker plots for arterial blood $\mathrm{pH}(\boldsymbol{A})$, partial arterial oxygen pressure (B) and partial arterial carbon dioxide pressure (C), with whiskers extending up to 1.5 times interquartile range. Outliers are displayed as open circles, with a random offset to limit visual overlap. 


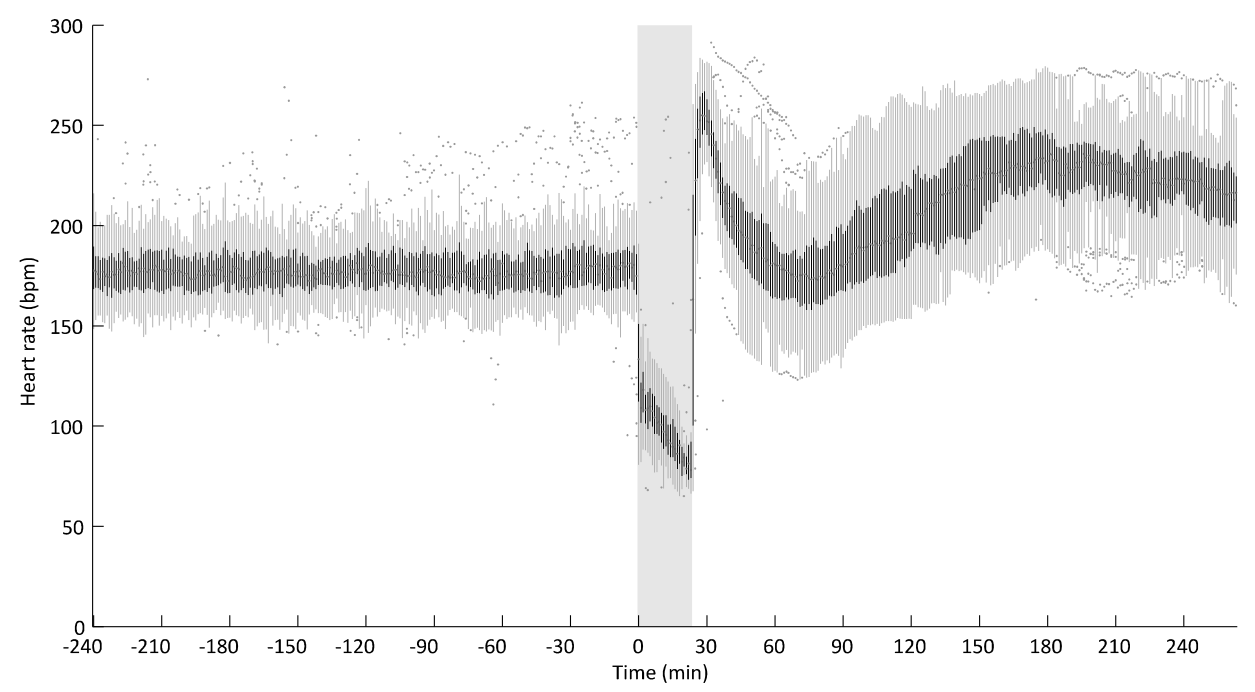

Figure 3.6: Whisker plot for heart rate with whiskers extending up to 1.5 times interquartile range. Outliers are displayed as open circles, with a random offset to limit visual overlap.
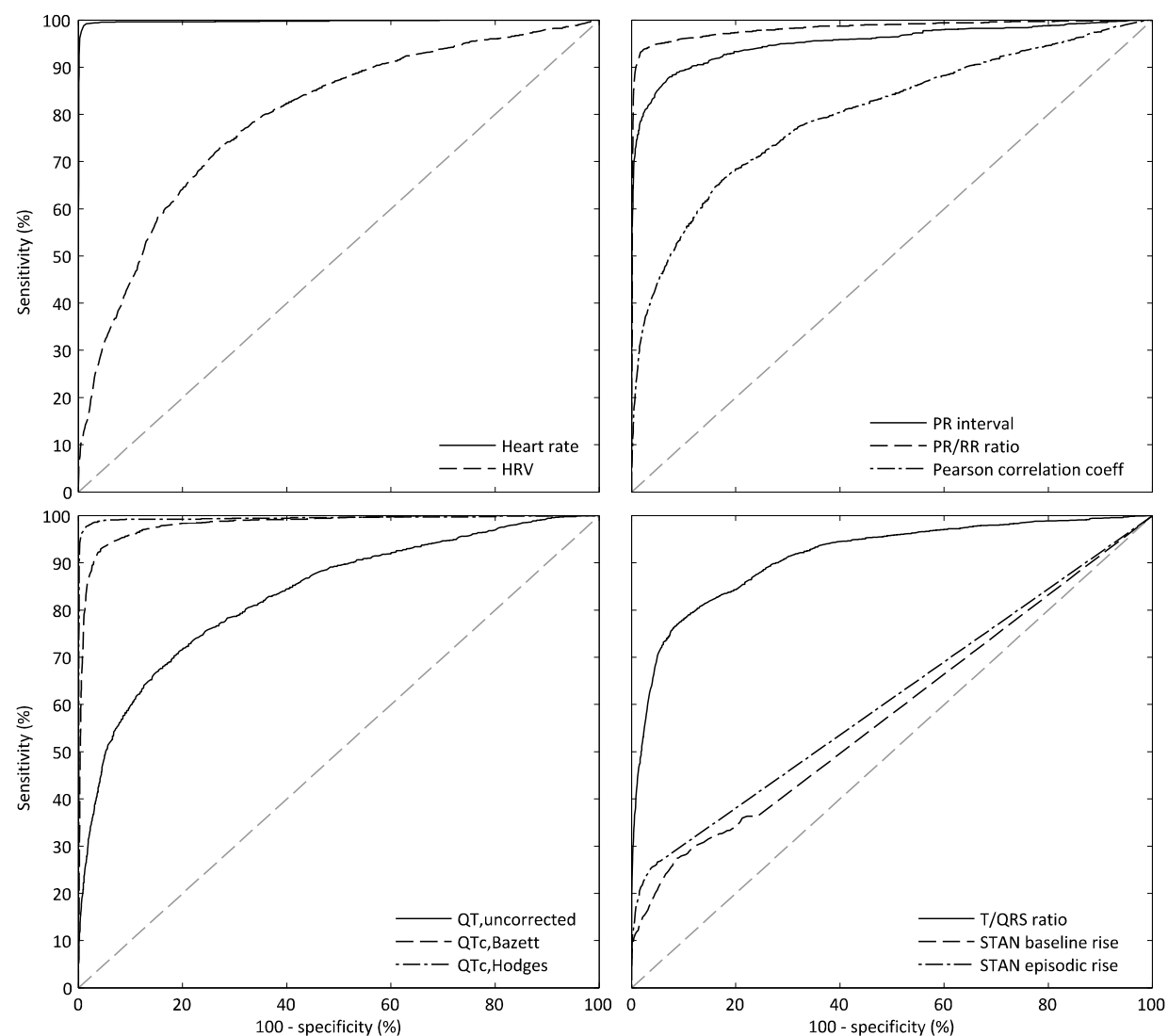

Figure 3.7: ROC curves for ECG markers for hypoxia. 

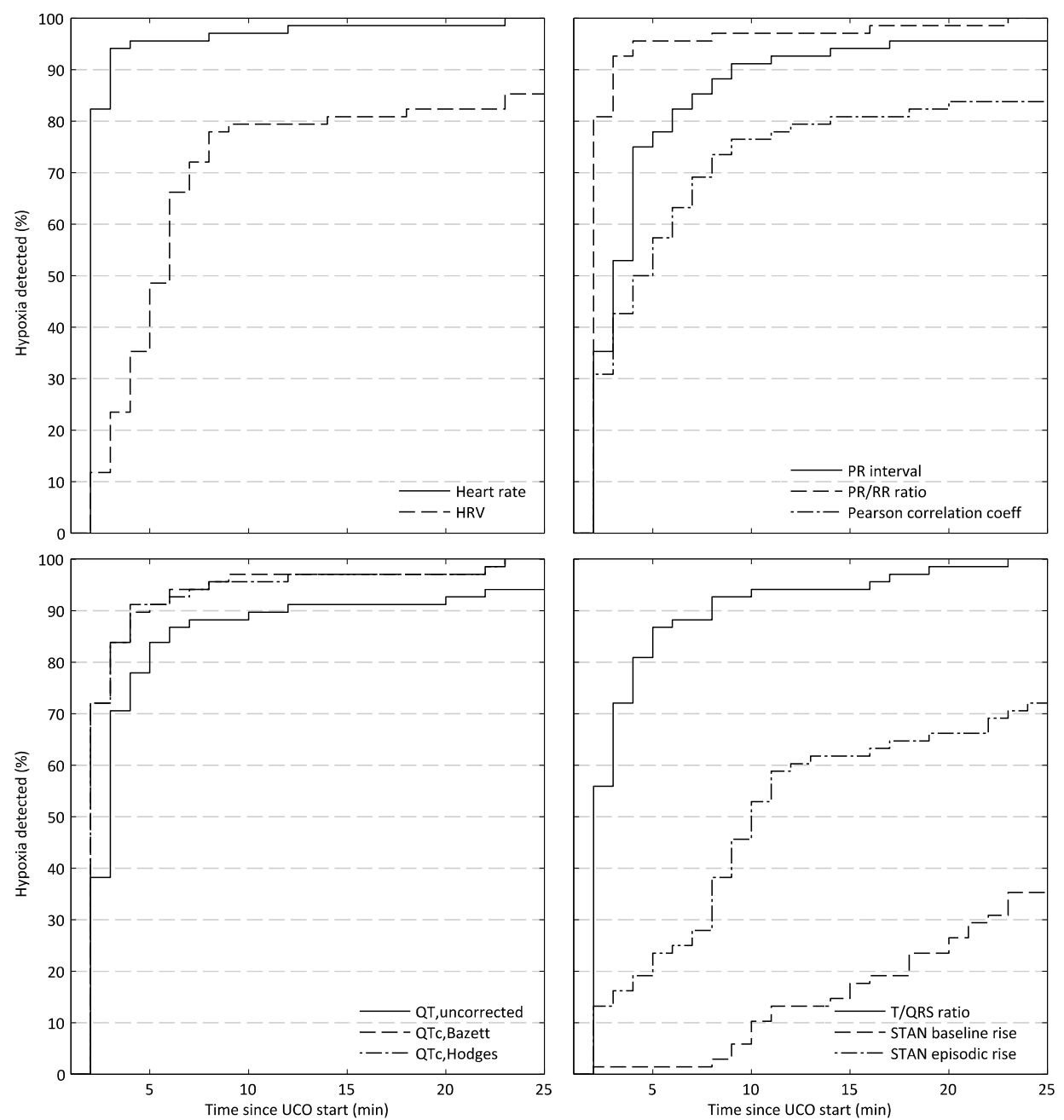

Figure 3.8: Detection plots for the percentage of fetuses in which hypoxia was detected during UCO.

\section{Discussion}

Interval-based ECG markers generally performed better than waveform-based markers in detecting hypoxia in a standardized preterm ovine model for severe hypoxiaischemia. The standardized period of complete UCO resulted in severe asphyxia which was characterized by acidosis. Hence, any potential clinically relevant marker was expected to deviate from baseline values at some point during UCO. Changes in interval-based heart rate, PR interval, PR/RR ratio and Bazett's and Hodges' corrected QT correlated strongly with hypoxia. In contrast, waveform-based STAN episodic and baseline rise markers were only weakly correlated.

Decreases in fetal heart rate with respect to baseline were found to be sensitive and specific for hypoxia in our model. Heart rate decelerations are a well-known effect of 
hypoxia, but can also be due to other factors, such as maternal movement, uterine contractions and fetal sleep cycles, limiting specificity of the marker in clinical practice ${ }^{31}$. Due to our experimental setup, the influence of non-hypoxic factors on heart rate was estimated to be less than within clinical settings, which led to near perfect behaviour based on the ROC curve.

Another interval-based marker studied was fetal heart rate variability. Depressed heart rate variability correlates to acidosis ${ }^{86}$. We found that HRV became severely depressed after onset of acidosis, as shown in Figure 3.9. This is reflected in the gradual increase in detection rate during the first 9 minutes after UCO start (Figure 3.8). Detection of hypoxia by the HRV marker was hampered due to substantial baseline variation, which is reflected in a low PPV of $34 \%$. This is most likely caused by our implementation of HRV. Significant correlation of HRV with hypoxia-ischemia was reported in literature ${ }^{87,88}$. Variation in HRV could be reduced by calculating the marker over a longer time period.

Changes in PR/RR ratio were found to be highly correlated to hypoxia. However the specificity of the PR/RR ratio as a marker for hypoxia and acidosis has been disputed ${ }^{89}$. While the decrease in PR/RR ratio during UCO was clear, Westgate et al. concluded that under conditions of no or only mild acidosis a decrease in PR/RR ratio was a marker of reflex responses to heart rate decelerations, and that it did not provide additional information concerning the metabolic reserves of the fetus ${ }^{89}$.

PR interval reflects atrioventricular delay and was found to strongly correlate with hypoxia. AV delay was generally increased during UCO (Figure 3.10). Others have reported a decrease in $A V$ delay due to hypoxia ${ }^{38,40}$. It is tempting to postulate that the different duration and number of hypoxic events are responsible for the observed differences. More precisely, a single long occlusion instead of a series of shorter occlusions was used in our study to induce hypoxia and acidosis. Atrioventricular delay may be increased due to severely depleted metabolic reserves during the single occlusion. Because the PR-marker seems to depend on the precise course of hypoxia, its clinical effectiveness is questionable. A similar observation can be made with regards to PR-HR correlation. For the PR-HR correlation marker $64 \%$ and $36 \%$ of alarms for hypoxia were due to respectively an increase above the positive threshold or a decrease below the negative threshold. Therefore, PR and PR-HR correlation are unlikely to be clinically effective markers, which is corroborated by the inconclusive results with regard to these markers found in the clinical trial by Strachan et al. ${ }^{90}$.

Changes in Hodges' and Bazett's corrected QT markers were found to be very sensitive and specific to hypoxia, and demonstrated high PPV as well. QT interval length was stable during the baseline period and decreased during hypoxia. This finding is supported by the observations of Oudijk et al. ${ }^{41}$, who demonstrated that $\mathrm{QT}_{\mathrm{c}, \mathrm{Bazett}}$ interval shortening did not depend on heart rate and was unrelated to normal labour stress. QT shortening may be linked to a catecholamine surge that is released when energy balance in the myocardium becomes negative due to hypoxia and anaerobic myocardial glycolysis starts ${ }^{41,91-93}$. The heart rate corrected QT interval markers may therefore be useful as specific markers for hypoxia.

Because clinical guidelines for STAN are specified for infants older than 36 weeks gestation it can be argued that STAN is not applicable to a preterm ovine model, such as 
the one used here. However, it was demonstrated that the response of the ST waveform to hypoxia is similar in preterm and term ovine fetuses ${ }^{73,74}$, and thus that ST waveform analysis can be reliably performed even in the preterm fetus.

However, STAN-device analogue episodic and baseline rise markers did not differ from normoxic baseline values in $65 \%$ and $28 \%$ of the fetuses at any point during UCO. The negligible correlation and low sensitivity for these markers indicate that these markers cannot be used to distinguish between hypoxia and normoxia. Our analysis did not provide evidence to support the hypothesis that STAN episodic and baseline markers are clinically useful. Whereas UCO in our model resulted in severe hypoxia and acidemia, in clinical practice perinatal hypoxia is commonly manifested through multiple shorter periods of hypoxia and is usually less severe. The fact that STAN episodic and baseline rise markers performed poorly under severe hypoxic conditions makes better performance under less severe conditions unlikely.

Waveform-based STAN episodic and baseline rise markers have already been implemented in a clinical device. The poor performance of these markers is thus surprising, yet supported by meta analyses of clinical trials ${ }^{35,36}$. As the name suggests, STAN episodic and baseline rise markers assume that hypoxia induces a rise in T/QRS ratio. In our model several subjects responded to hypoxia by developing more negative T waves (Figure 3.11). This is ignored by focusing solely on positive T/QRS ratio changes.

A general disadvantage of waveform-based markers is that the shape of the ECG waveform depends on the orientation of the ECG lead with respect to the heart vector (Figure 3.1). Differences in lead orientation, differences in cardiac anatomy and differences in cardiac response to hypoxia between individuals cause variance in waveform-based marker values. Interval-based markers do not rely directly on the shape of ECG waveforms. Therefore, interval-based markers are hypothesized to provide more stable performance than waveform-based markers.

As mentioned above, fetuses in this study were exposed to profound hypoxiaischemia. Because profound hypoxia-ischemia should produce a clear change in any potential marker for hypoxia, our model allowed evaluation and comparison of markers to identify potential markers for hypoxia. Generally, the clinical onset of hypoxia is gradual. In clinical cases the baseline period is harder to define and heart rate patterns are far less predictive ${ }^{75}$. We expect that marker performance in clinical cases will be lower than that reported here.

\section{Conclusion}

The most suitable ECG markers for detecting fetal hypoxia were interval-based, rather than waveform-based. Interval-based markers heart rate and heart rate corrected QT length were able to detect fetal hypoxia in our standardised ovine model and constitute promising markers for detecting hypoxia in human fetuses. In contrast, waveform-based STAN episodic and baseline T/QRS rise markers, which were our analogue of the markers implemented in clinical STAN devices, could not assess severe hypoxia-ischemia. 


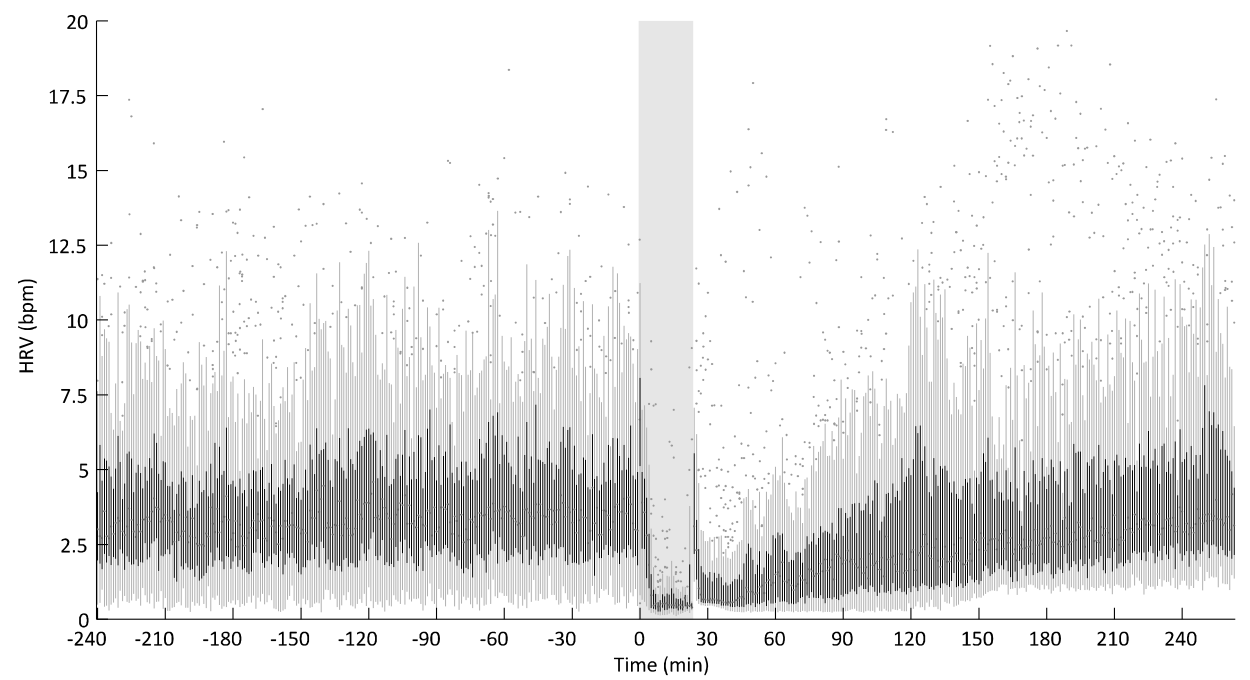

Figure 3.9: Whisker plot for heart rate variability with whiskers extending up to 1.5 times interquartile range. Outliers are displayed as open circles, with a random offset to limit visual overlap.

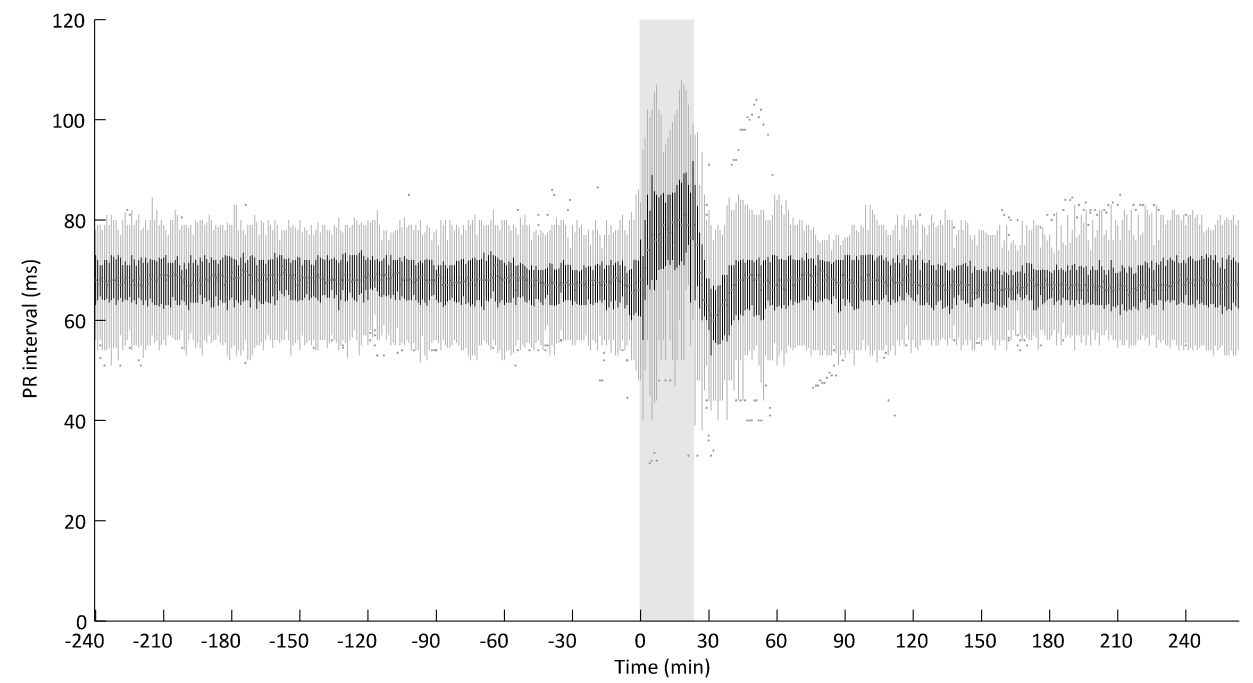

Figure 3.10: Whisker plot for the PR interval with whiskers extending up to 1.5 times interquartile range. Outliers are displayed as open circles, with a random offset to limit visual overlap. 


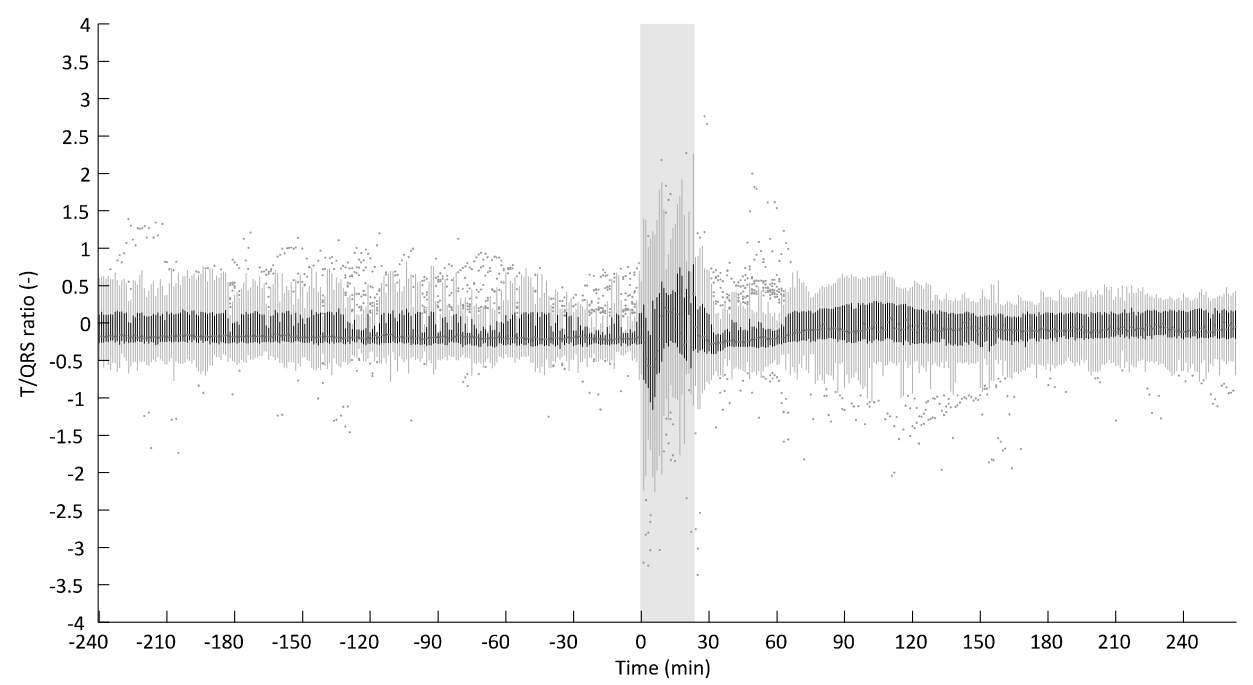

Figure 3.11: Whisker plot for the T/QRS ratio with whiskers extending up to 1.5 times interquartile range. Outliers are displayed as open circles, with a random offset to limit visual overlap. 



\section{Detection of short seizures}

Published as:

Zwanenburg, A., Andriessen, P., Jellema, R. K., Niemarkt, H. J., Wolfs, T. G. A. M., Kramer, B. W. \& Delhaas, T. Using trend templated in a neonatal seizure algorithm improves detection of short seizures in a fetal ovine model. Physiol Meas 36, 369-384 (2015) 



\section{Abstract}

Background Seizures below one minute in duration are difficult to assess correctly using seizure detection algorithms. We aimed to improve neonatal detection algorithm performance for short seizures through the use of trend templates for seizure onset and end.

Methods Bipolar EEG were recorded within a transiently asphyxiated ovine model at 0.7 gestational age, a common experimental model for studying brain development in humans of 30-34 weeks of gestation. Transient asphyxia led to electrographic seizures within 6-8 hours. A total of 3159 seizures, 2386 shorter than one minute, were annotated in 1976 hour-long EEG recordings from 17 fetal lambs. To capture EEG characteristics, five features, sensitive to seizures, were calculated and used to derive trend information. Feature values and trend information were used as input for support vector machine classification and subsequently post-processed. Performance metrics, calculated after post-processing, were compared between analyses with and without employing trend information. Detector performance was assessed after five-fold crossvalidation conducted ten times with random splits.

Results The use of trend templates for seizure onset and end in a neonatal seizure detection algorithm significantly improves the correct detection of short seizures using two-channel EEG recordings from 54.3\% (52.6-56.1) to 59.5\% (58.5-59.9) at FDR 2.0 (median (range); $p<0.001$, Wilcoxon signed rank test).

Conclusion Using trend templates may aid in detection of short seizures by EEG monitoring at the NICU. 


\section{Introduction}

Computer-assisted detection of neonatal seizures may help the clinician to detect neurological dysfunction that would otherwise go unnoticed ${ }^{45,94}$. Electrographic seizures are usually marked by distinct changes in the EEG, compared to background signal ${ }^{17,95}$. Seizure detection algorithms use one or more characteristics - or features-of the EEG signal to determine seizure presence. Following developments in the machine learning field and concurrent increases in computing power over the last decades, development of algorithms has progressed. In general, algorithms have become more complex and detector performance has improved through the implementation of machine learning algorithms such as neural networks ${ }^{50}$ and support vector machines (SVM) ${ }^{49}$. However, especially for short seizures ( $<1$ minute) improving the trade-off between a high good detection rate (GDR) and low false alarm rate is still a challenge ${ }^{49}$.

The detection challenge can be more broadly formulated as the ability to distinguish seizure activity from non-seizure background. The ability to distinguish between seizure and non-seizure activity depends primarily on the quality of classifier input, i.e. the sensitivity of features to seizure and non-seizure activity. Post-processing after classification further refines the ability, yet its effectiveness still depends on classifier input quality. Thus, our approach to the detection challenge posed by short detections is to increase the quality of classifier input.

Neonatal detection algorithms are currently based on interpreting feature values calculated within a short time window of several seconds and do not directly take information contained in neighbouring epochs into account. We hypothesize that incorporating trend information of seizure onset and end into epoch classification leads to improved good detection rates. We first derive and then implement trend templates for seizure onset and end at the feature level. Subsequently this trend-based information is included as an additional feature for SVM classification.

Our group has previously used two-channel EEG, recorded in transiently asphyxiated preterm fetal sheep of 0.7 gestational age (GA; 102 days, term 146 days), to evaluate post-asphyctic seizure burden for treatment effects ${ }^{69,70}$. The fetal ovine model is often used in studies on brain development. In terms of brain development $0.7 \mathrm{GA}$ preterm sheep are broadly equivalent to the preterm human infant of 30-34 weeks, before the onset of cortical myelination ${ }^{96,97}$. Background patterns ${ }^{56,98}$ and pathological features, such as seizures, in ovine fetal EEG recordings are similar to those observed in humans ${ }^{26,30}$. Using complete recordings from 17 preterm sheep, we were able to construct a suitable EEG database containing predominantly (75.5\%; 2386 of 3159) short seizures and homogenous seizure counts between subjects. Seizure detection performance was assessed by iterating over the dataset ten times using five-fold crossvalidation. 


\section{Methods}

\section{Animal experiment}

Animals The study group consisted of 17 fetal sheep carried by 17 date-mated Texel ewes. Fetal instrumentation was performed at $0.7 \mathrm{GA}$. The experiments were approved by the Animal Ethics Research Committee of Maastricht University, the Netherlands.

Experiment The animals were instrumented according to the procedures described by Jellema et al. ${ }^{29}$. In short, an incision was made in the uterus and an inflatable vascular occluder (OC16HD, 16 mm, In Vivo Metric, Healdsburg, CA, USA) was placed around the umbilical cord. After laying bare the fetal skull, four holes were bored into the cranium, through which two pairs of silver-tipped EEG electrodes (Cooner Wire Co., Chatsworth, CA, USA) were placed bilaterally on the dura over the parasagittal parietal cortex ( $5 \mathrm{~mm}$ and $15 \mathrm{~mm}$ anterior to point bregma and $10 \mathrm{~mm}$ lateral). EEG electrode placement was similar to the placement of $\mathrm{C} 3, \mathrm{C} 4$ and P3 and P4 electrodes in the reduced neonatal 1020 system. The EEG electrodes were secured with cyanoacrylate glue and fetal skin was replaced over the cranium and sutured. Subsequently, the uterus was closed, and leads were extracted through a trocar hole in the flank of the ewe. Fetus and ewe were allowed to recover for 4 days. At the fourth day of experiment, the occluder was inflated and the fetus was subjected to 25 minutes of umbilical cord occlusion (UCO), leading to acute global hypoxia-ischemia and cerebral hypoperfusion, and the occurrence of seizures after several hours ${ }^{26,99}$. The occurrence of seizures after hypoxiaischemia is consistent with that in the human newborn ${ }^{58}$. Ewe and fetus were euthanized 7 days after UCO by administration of pentobarbital.

\section{Data acquisition}

Data were acquired from the two bipolar EEG leads and digitized by an MPAQ unit (Maastricht-Programmable AcQuisition system, Maastricht Instruments BV, Maastricht, the Netherlands), sampled at $1000 \mathrm{~Hz}$ and subsequently stored on a hard disk drive for off-line analysis. Data were continuously recorded post-UCO until euthanization.

Preprocessing Data were processed off-line using MATLAB R2013a (The MathWorks, Inc., Natick, MA). EEG data were filtered using a $50 \mathrm{~Hz}$ notch filter and $0.5-30 \mathrm{~Hz} 4$ thorder Butterworth bandpass filter to remove mains noise and unwanted signal components. To facilitate further data processing, data were downsampled to $250 \mathrm{~Hz}$. Flat-line and high voltage $(>1000 \mu \mathrm{V})$ artefacts were detected and removed. Seizures were subsequently annotated by $A Z$, based on continuous EEG supported by simultaneous amplitude-integrated EEG $(\mathrm{aEEG})^{16}$, and according to recommendations for seizure morphology ${ }^{17,56,95}$. Both annotations and EEG were reviewed by RJ and PA, who, as a trained neonatologist with experience in two-channel EEG/aEEG interpretation, did the final interpretation. EEG recordings were divided into 1976 hourlong segments containing a total of 3159 annotated seizures of median 41.4 (interquartile range: $30.5-59.3$ ) seconds in duration.

Standard features Five features, each describing a characteristic of EEG recordings with established sensitivity to seizure activity, were selected for implementation: line length ${ }^{100,101}$, non-linear energy ${ }^{101,102}$, sample entropy $y^{51,103}$, aEEG lower bound ${ }^{94}$ and $6^{\text {th }}$ 
sublevel wavelet coefficient ${ }^{101,104}$. Recordings were divided into 8 second epochs with $50 \%$ overlap, on the basis of recommendations by the International Federation of Clinical Neurophysiology for interpretation of electrographical seizures with an abnormal EEG background ${ }^{49,105}$. Feature values were calculated for each epoch in each of the two EEG channels. The set of feature values in each epoch forms a feature vector $\vec{x}$.

Our detection algorithm used a support vector machine (SVM) as classifier. SVM classification accuracy is influenced by feature scaling and robustness of input data. To improve SVM classification accuracy, feature values were rescaled by subtraction of their median value and subsequent division by the difference between values of percentiles P95 and P5 over the entire dataset.

\section{Algorithm validation}

As shown in Figure 4.1, validation of the neonatal seizure detection algorithm was divided into three stages: SVM classifier training, post-processing optimization and independent validation. We used five-fold cross-validation with stratified folds to assess detector algorithm performance. We assigned three of the five folds to SVM classifier training, one fold to post-processing optimization and one fold to independent validation, which led to twenty unique fold permutations. Cross-validation was repeated ten times using different splits to arrive at a more stable Monte Carlo estimate for detector performance ${ }^{106}$.

Folds were created by randomly assigning hour-long EEG recordings. Fold stratification was conducted by comparing proportions of seizure and non-seizure labels within each fold to the proportion of labels within the complete data set. The split into folds was accepted when both the proportion of labels within each fold was within $5 \%$ percent of the expected proportions based on the complete data set, and the number of labels within each fold was within $5 \%$ percent of each other. Otherwise the split of EEG recordings into folds was rejected and the procedure repeated until an acceptable split was found.

SVM classifier training To classify feature vectors we used an SVM, which maps feature vectors from input space to a higher-dimensional space to determine an optimal separation between classes. An example is shown in Figure 4.2.

We used the LIBSVM implementation ${ }^{107}$ of SVM with the Radial Basis Function kernel. SVM classifier training, necessary to enable classification of epochs in postprocessing optimization and independent validation folds, was conducted using annotated epochs in the classifier training fold. To reduce model overfitting risk, nonseizure epochs were randomly selected to create a set similar in size to the set of seizure epochs. The Radial Basis Function kernel required two input parameters, namely soft margin parameter $C$ and kernel parameter $\gamma$, which determined how strictly the SVM model fitted input feature vectors. Input parameter values were initially optimized by performing a grid search with ten-fold cross-validation ${ }^{108}$. Further refinement of input parameters $C$ and $\gamma$ was conducted by employing a Nelder-Mead algorithm in the neighbourhood of the initially optimized parameter values. 

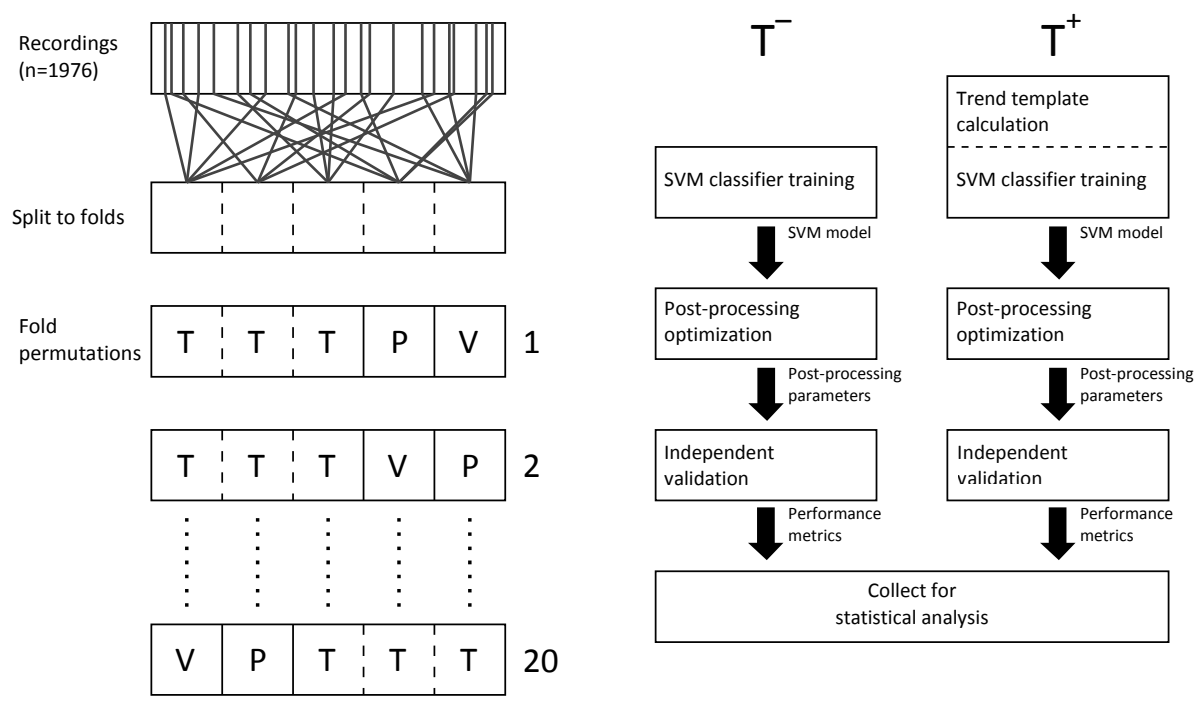

Figure 4.1: Five-fold cross-validation and algorithm evaluation and analysis. (A) 1976 recordings were randomly assigned to five folds. In each fold permutation three folds are assigned for SVM classifier training $(T)$, one fold is assigned for post-processing optimization $(P)$ and one fold for independent validation (V). The total amount of unique fold permutations is twenty. (B) The analysis procedure for each fold permutation involved several processing steps. $T$ and $T^{+}$respectively designate analysis without and with quantification of feature behaviour over time by using trend templates. Initially, an SVM model was built using recordings assigned to the training folds. For the $T^{+}$set trend templates for seizure onset and end were calculated and subsequently incorporated as an additional SVM input feature before SVM training occurred. Subsequently, the SVM model was used to classify data from recordings assigned to the post-processing optimization fold. Post-processing optimization yielded a set of post-processing parameters which produced an optimal good detection rate. Both the SVM model and post-processing parameters were then used in classification and post-processing of data in the independent validation set. Performance metrics were calculated based on resulting detector decisions and expert annotations and collected for further analysis. 


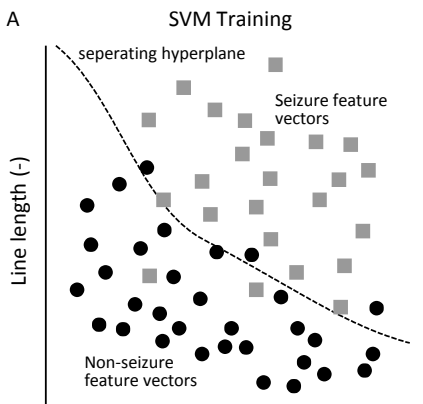

Wavelet coeff. (-)

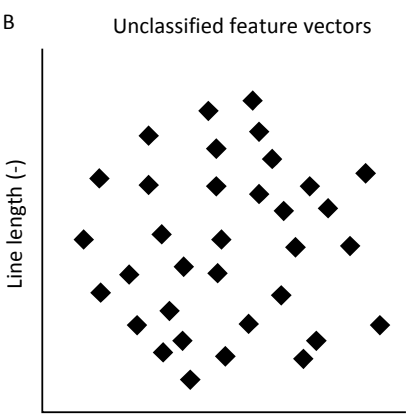

Wavelet coeff. (-)

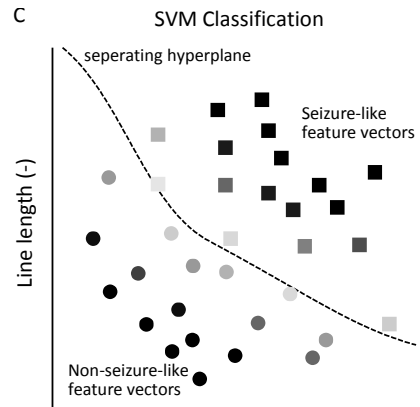

Wavelet coeff. (-)

Figure 4.2: Support vector machine (SVM) training and classification: (A) During training, feature vectors annotated as occurring during seizure epochs (squares) and non-seizure epochs (circles) are used by the SVM to find an optimally separating hyperplane (dashed line) through corresponding support vectors (not indicated). These support vectors are stored in a model to classify new data. (B) Unclassified feature vectors (rhombus) such as those of epochs in the post-processing optimization and independent validation folds. (C) Feature vectors from (B) are classified using the model trained in (A). In this case, due to the specific SVM kernel chosen, classification is not strictly binary. Features vectors are classified as seizure-like (squares) and non-seizurelike (circles). Grading indicates that feature vectors distant from the hyperplane are more likely to correspond to seizure or non-seizure epochs.

For post-processing purposes binary classification into seizure or non-seizure classes by SVM is undesirable, and it is preferable that epochs should be assigned class probabilities instead. SVM output, however, does not take the form of probabilities and needs to be converted. We used sigmoid fitting, introduced by J. Platt ${ }^{109}$, for SVM output conversion. An adaptation was made by Lin et al. ${ }^{110}$ and implemented within the LIBSVM package. An SVM model-specific two-parameter sigmoid function was fitted to the SVM output of the training folds and parameter values for the sigmoid function were estimated.

Post-processing optimization SVM classification yields class probabilities for single epochs, whereas most seizures in reality span multiple sequential epochs. To adequately arrive at a classification decision for seizure and non-seizure events, it is necessary to post-process sequences of epochs. Post-processing involves multiple steps and thus multiple parameters that are not a priori known. Values of post-processing parameters have to be learned through an optimization process. This optimization is performed on data assigned to the post-processing optimization fold.

Post-processing steps Post-processing steps were based on the method described by Temko et al. ${ }^{49}$ and are shown in Figure 4.3. After SVM classification, conversion of SVM output into empirical probabilities was conducted using the sigmoid function derived during classifier training. To arrive at a final decision, empirical probabilities for each channel were further post-processed as described below. Please note that parameter values for post-processing steps will be determined during optimization. 
1. Moving average filtering: Probabilistic output for each EEG channel was filtered using a moving average filter of order $n$.

2. Compare against threshold: After filtering, resulting epoch values pepoch were compared against a threshold value $p_{\text {threshold }}$. Epochs were assigned to the seizure-like class (or ' 1 ') if pepoch $\geq$ pthreshold or non-seizure-like class (' 0 ') if $p_{\text {epoch }}<p_{\text {threshold }}$.

3. Combine channels: Thresholded output for both channels was combined using a logical 'OR' operation.

4. Join neighbouring seizure-like segments: Seizure-like segments were joined when they were within $m$ epochs of each other.

5. Collar seizure-like segments: Seizure-like segments were collared to include $k$ epochs at the start and end of each segment.

6. Remove seizure-like segments that were too short: This post-processing step was an addition to the scheme proposed by Temko et al. ${ }^{49}$. One method for false detection reduction is demanding that detections have a minimum duration ${ }^{111}$. Hence seizure-like segments shorter than $h$ epochs in duration were classified as non-seizure-like.

Post-processing parameter optimization Good detection rate (GDR), being the percentage of annotated seizures correctly detected by the algorithm, was used as the optimization target for optimizing post-processing parameter values for steps 1, 2, 4, 5 and 6 . It was necessary to impose constraints during the optimization process to prevent optimizing towards artificially high GDR and low FDR. Achieving high GDR with a low number of false detections is arbitrarily easy with post-processing parameter values chosen such that most epochs are classified as seizure. In this situation, a single false detection will be found in a recording without any seizures present, whereas in recordings with one or more annotated seizures present all annotated seizures are detected, leading to both high GDR and low FDR. To avoid this situation, we imposed two constraints during optimization. The first constraint limited mean false detection duration to 2.5 minutes, close to the mean false detection durations found by Temko et al. in their post-hoc analysis ${ }^{112}$. The second constraint was to evaluate GDR at set false detection rates (FDR), namely $0.50,1.00$ and 2.00 false detections per hour. Evaluating GDR at set FDR furthermore served to facilitate comparison of performance metrics between fold permutations, Monte Carlo iterations and other detection algorithms. Parameter combinations yielding the highest GDR within the imposed constraints were used for analysis of the independent validation fold. 

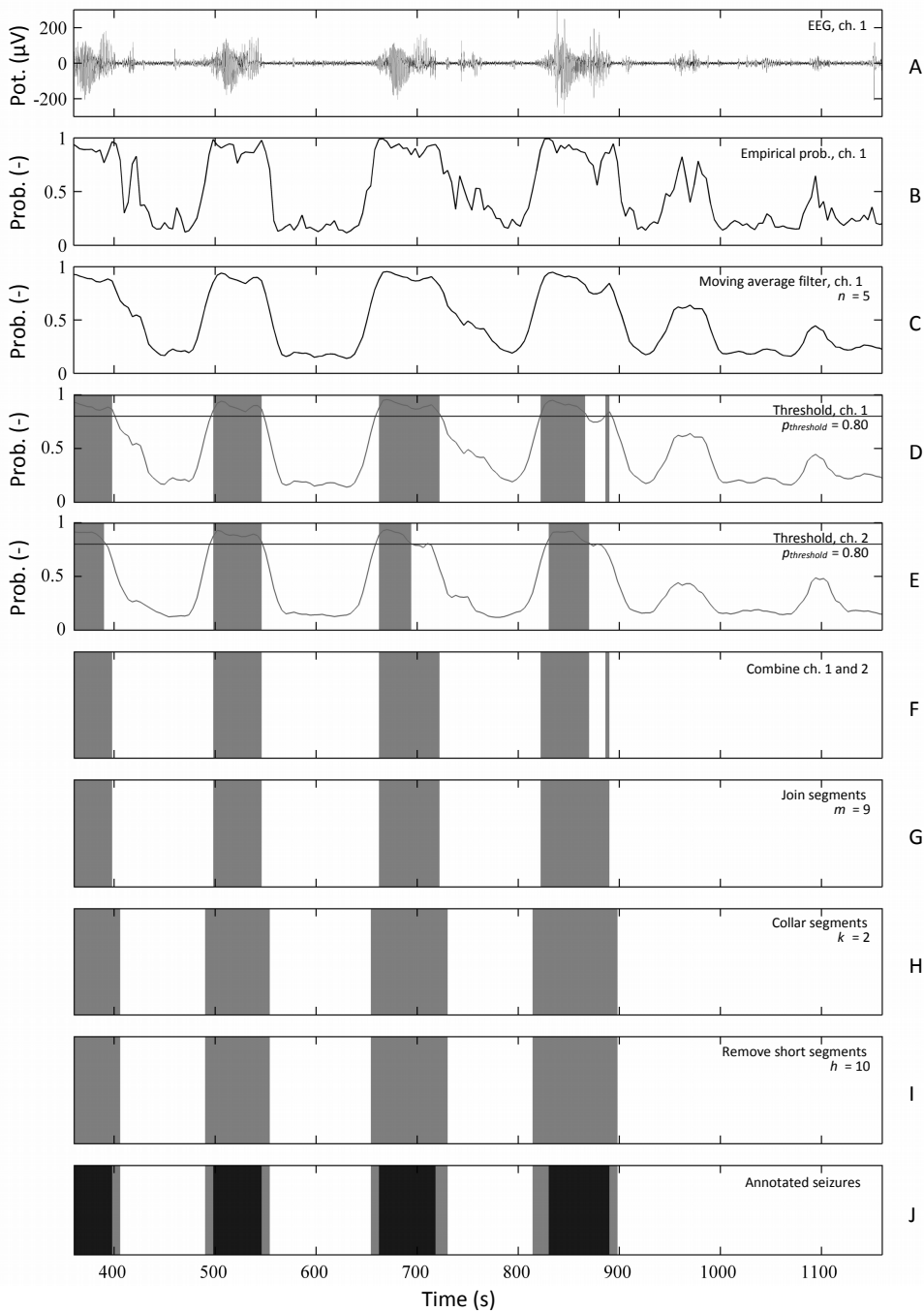

Figure 4.3: Example of post-processing steps, with typical values for post-processing parameters: (A) EEG recording in channel 1. (B) Empirical probabilities for channel 1, after conversion of SVM output. (C) Empirical probabilities for channel 1 after filtering with a 5 th order moving average filter. (D) Seizure-like segments (grey rectangles) after applying a threshold (grey line) for channel 1. Empirical probabilities are present in the background. (E) Seizure-like segments after applying a threshold for channel 2. Note that preceding processing steps for channel 2 are not shown. (F) Seizure-like segments after channel combination through a logical 'OR' operation. (G) Seizure-like segments after joining other segments within a 9 epoch radius. (H) Seizure-like segments after collaring segments by 2 epochs. (I) Seizure-like segments after removing segments shorter than 10 epochs. Note that in the example, this has no effect. (J) Detected seizures compared with annotated seizure (black). 
Independent validation Performance assessment was conducted on the data assigned to the independent validation set. After applying the SVM model to the independent validation set and converting the SVM output to empirical probabilities, post-processing was performed using parameter combinations derived during optimization. Performance metrics were subsequently calculated on the post-processed data.

Performance metrics We report epoch-based and event-based metrics as in Temko et al. ${ }^{112}$ by comparing detection output from the validation set with expert annotation. $A$ confusion matrix (also known as a contingency table or an error matrix) was constructed by comparing the annotations of epochs with decisions made by the algorithm. From the confusion matrix, epoch-based metrics sensitivity, specificity, and precision were calculated. GDR for all seizures, GDR for seizures shorter than 1 minute and the realised-in contrast to imposed-FDR are reported as event-based metrics. In addition mean false detection duration is reported.

\section{Quantifying feature behaviour over time}

Trend templates Feature behaviour over time was quantified using trend templates for seizure onset and end, as shown in Figure 4.4. During the onset of an electrographic seizure, feature vectors exhibit a typical transition from non-seizure background to seizure state. A comparable transition is observed at seizure end. Both transitions can individually be linearized over time to create a trend template for each transition. A trend template describes the mean slope during state transition as well as the mean duration of the transition for each feature. The latter defines the template width.

For the onset transition we derived the template width for each feature as well as the corresponding slope as follows. Electrographic seizures were previously annotated in recordings assigned to the SVM classifier training folds. The transition of each individual feature was studied within a window of 12 epochs centred on the annotated seizure onset. The template width for each feature was determined by assessing the length of segments of interest (SOI) over all the onsets in the training folds. SOI were defined as follows. If the transition from non-seizure state to seizure state was characterized by an increase in feature value, the SOI was the segment of consecutive epochs which possessed three characteristics: it was monotonously increasing, contained the maximum rate of change within the segment and was the longest segment where the feature value is monotonously increasing. In case the transition from non-seizure to seizure state was characterized by a decrease in feature value, SOI were assumed to be monotonously decreasing instead of increasing. The mean length of all SOI within the training folds was used as the template width for the feature in question, leading to a typical template width of 5 epochs for every feature.

Subsequently the slope for each feature was determined as follows. As before we determined SOI within the 12 epoch-wide window centred on the onset, but shrunk or extended these segments to match the corresponding template width in length. The resulting sequence of feature values was linearized and the value of the slope stored. The mean value of slopes in SOI within the training folds was used as template slope.

The trend template for the transition from seizure to non-seizure state was derived as described above, but with the assumption that SOI were monotonously decreasing 
or increasing if feature values during seizure state are respectively higher or lower than during non-seizure state.

After template derivation for each of the five features, templates were used to quantify temporal changes in feature values by calculating scalar resolutes. We defined the displacement vector $\vec{v}(t)=\left\{w_{i}^{-1} \cdot\left(x_{i}(t)-x_{i}\left(t-w_{i}\right)\right) \mid i=1, \ldots, 5\right\}$ where $x_{i}(t)$ was the value for feature $i$ at epoch $t$ and $w_{i}$ the corresponding template width. For the onset template let $\vec{y}=\left\{y_{i} \mid i=1 \ldots 5\right\}$ be the template vector with $y_{i}$ the template slopes for individual features. The corresponding scalar resolute was $s_{1}(t)=(\vec{v}(t) \cdot \vec{y}) /|\vec{y}|$. The same calculation was performed for the seizure end template, yielding the scalar resolute $s_{2}(t)$.

$s_{1}$ and $s_{2}$ demonstrated alternating behaviour during seizures. Since features should be able to distinguish between seizure and non-seizure classes and alternating behaviour prevents this, direct implementation of scalar resolutes as separate features was undesirable. Instead we used an envelope function of the maximum value of the scalar resolutes. Let $s_{\max }(t)=s_{1}(t) \vee s_{2}(t)$. The envelope function was defined as $h(t)=\left(\frac{2}{N} \sum_{k=0}^{N-1} s_{\max }(t-\lfloor N / 2\rfloor+k)^{2}\right)^{1 / 2}$, where $N$ was the maximal template width in epochs. Subsequently, $h(t)$ was normalized according to the same procedure as other features and used as an additional feature in SVM training and classification.

Implementation To assess the performance effects of using trend templates to quantify feature behaviour over time, we performed the analysis as described in the section concerning algorithm validation twice. The first analysis was based solely on the set of five features described earlier, and will be referred to as $\mathrm{T}^{-}$. In addition to the method described in the section concerning algorithm validation, the second analysis involved calculating trend templates in the training folds and subsequent derivation of the envelope functions in every fold as an additional feature. Hence, the feature set for SVM training and classification was extended from five to six features. The corresponding analysis is referred to as $\mathrm{T}^{+}$. Note that both data and fold assignment were the same for $\mathrm{T}^{-}$and $\mathrm{T}^{+}$at every step of the analysis.

\section{Statistical analysis}

In this study we assessed the performance effects of quantifying feature behaviour through the use of trend templates. Five-fold stratified cross-validation, iterated ten times with randomized data splits, was used as the analysis procedure, and performance metrics were calculated for each fold permutation in cross-validation. Mean values from the twenty permutations within each iteration are used as mean performance metrics. Statistical analysis is performed on the collection of mean performance metrics.

Following the recommendation by J. Demšar ${ }^{113}$, we used the non-parametric Wilcoxon signed ranks test to compare average performance metrics between $\mathrm{T}^{-}$and $\mathrm{T}^{+}$ sets. Differences in performance metrics were assumed significant at the $p=0.05$ level. 
A Original EEG

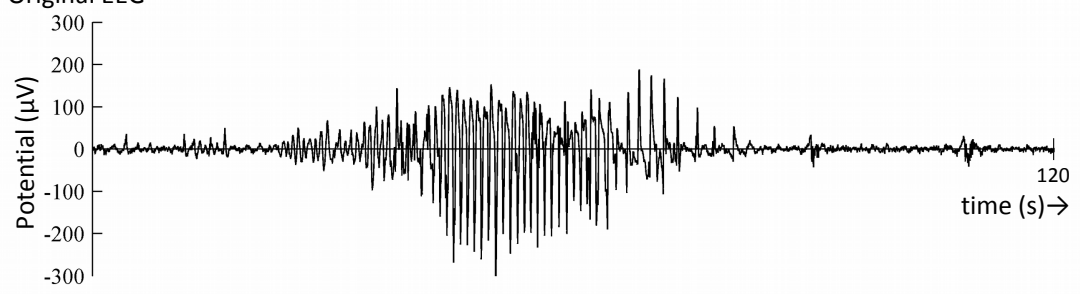

B Features and trend template calculation

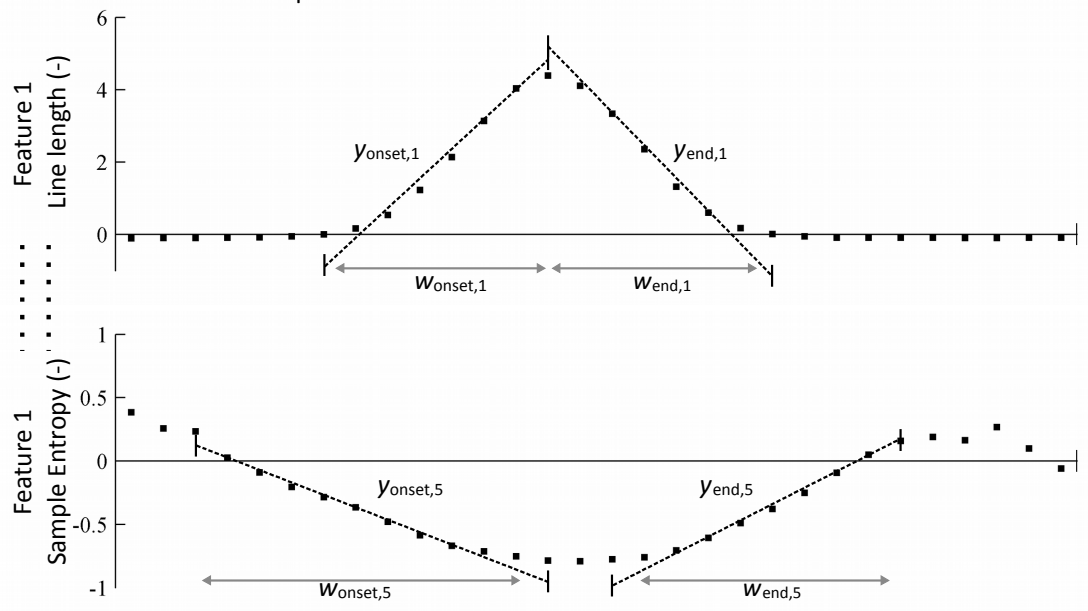

C Envelope function calculation

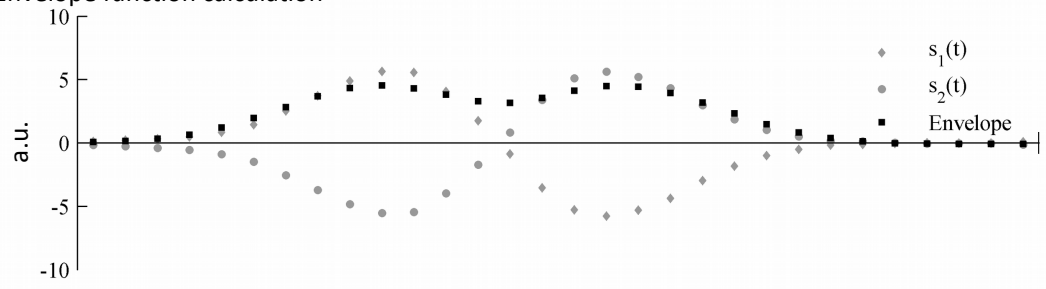

Figure 4.4: Quantifying feature behaviour through the use of trend templates. (A) Original single-channel EEG readout of an electrographic seizure. (B) Feature values for line length (top panel) and sample entropy (bottom panel) are calculated from the EEG readout within half-overlapping $8 \mathrm{~s}$ windows. The three other features are omitted from this example. Two trend templates for seizure onset and end for each feature are trained during SVM classifier training. The templates capture feature behaviour over time and consist of width $w$ and slope $y$. Trend templates are used to calculate scalar resolutes $s_{1}$ and $s_{2}$, for onset and end respectively, after training. High positive scores indicate that feature behaviour is similar to the calculated template. (C) Scalar resolutes are used to calculate an envelope function, which serves as an additional feature to quantify feature behaviour.

\section{Results}

We compare performance metrics between $\mathrm{T}^{-}$and $\mathrm{T}^{+}$sets for each imposed FDR (0.50, 1.00 and 2.00 false detections per hour). Performance metrics and comparisons are shown in Table 4.1. 
The constraints imposed during post-processing optimization yield consistent results for the independent validation set. Metrics derived from the independent validation set are consistently reproduced close to the imposed FDR values. False detection rates are consistent between $\mathrm{T}^{-}$and $\mathrm{T}^{+}$and mean false detection durations remained below the threshold of 2.5 minutes in each instance. This implies that performance metrics can be reliably compared between $\mathrm{T}^{-}$and $\mathrm{T}^{+}$.

Both overall GDR and GDR for short seizures improve significantly $(p<0.001)$ by quantifying feature behaviour using trend templates. GDR for short seizures improves from $21.4 \%$ to $30.6 \%$, from $34.8 \%$ to $43.0 \%$ and from $54.3 \%$ to $59.5 \%$ for imposed FDR of $0.50,1.00$ and 2.00 respectively. GDR thus increases through the use of trend templates, but the effect decreases with increasing FDR. Similar behaviour occurs for both sensitivity and precision.

A small, but significant $(p<0.01)$, decrease in specificity occurs in the $\mathrm{T}^{+}$set for imposed FDRs of 1.00 and 2.00. The decrease in specificity is due to the fact that seizures, as detected by the algorithm, are longer than corresponding annotated seizures, as is signified by relatively low precision. Since an increase in GDR is equivalent to an increase in the number of detected seizures, comparatively more epochs, annotated as non-seizure, are classified as seizure epochs in the $\mathrm{T}^{+}$set, leading to a decrease in specificity.

We analysed undetected seizures by assigning these to one of three categories, as shown in Table 4.2. The categories are: (I) Filtered empirical probabilities remained below detection threshold, i.e. rejected at post-processing step 2; (II) Seizure-like segment was too short, i.e. rejected at post-processing step 6; (III) Overlap with start or end of recording. Examples of seizures from each category can be seen in Figure 4.5. Minor differences in distribution of undetected seizures in categories are present between $\mathrm{T}^{-}$and $\mathrm{T}^{+}$sets. The majority of undetected seizures fall into category I, then category II, which indicates that these seizures were not sufficiently distinguishable from background activity at the imposed FDRs. 


\begin{tabular}{|c|c|c|c|c|c|c|}
\hline \multirow{2}{*}{$\begin{array}{l}\text { Set FDR }\left(^{-1}\right) \\
\text { Data set }\end{array}$} & \multicolumn{2}{|c|}{0.50} & \multicolumn{2}{|c|}{1.00} & \multicolumn{2}{|c|}{2.00} \\
\hline & $\mathrm{T}^{-}$ & $\mathrm{T}^{+}$ & $\mathrm{T}^{-}$ & $\mathrm{T}^{+}$ & $\mathrm{T}^{-}$ & $\mathrm{T}^{+}$ \\
\hline $\begin{array}{l}\text { GDR (\%) } \\
\text { overall }\end{array}$ & $\begin{array}{c}24.0 \neq \\
(21.8- \\
26.9)\end{array}$ & $\begin{array}{c}34.4 \neq \\
(32.5- \\
35.7)\end{array}$ & $\begin{array}{c}38.5 \neq \\
(36.4- \\
40.6)\end{array}$ & $\begin{array}{c}47.1 \neq \\
(46.2- \\
49.1)\end{array}$ & $\begin{array}{c}58.9 \neq \\
(57.4- \\
60.0)\end{array}$ & $\begin{array}{r}63.7 \neq \\
(62.7- \\
64.4)\end{array}$ \\
\hline $\begin{array}{l}\text { GDR (\%) } \\
<1 \mathrm{~min}\end{array}$ & $\begin{array}{c}21.4 \ddagger \\
(18.8- \\
23.8)\end{array}$ & $\begin{array}{c}30.6 \neq \\
(29.3- \\
31.8)\end{array}$ & $\begin{array}{c}34.8 \neq \\
(32.2- \\
36.1)\end{array}$ & $\begin{array}{r}43.0 \neq \\
(42.3- \\
44.4)\end{array}$ & $\begin{array}{c}54.3 \neq \\
(52.6- \\
56.1)\end{array}$ & $\begin{array}{r}59.5 \neq \\
(58.5- \\
59.9)\end{array}$ \\
\hline Specificity (\%) & $\begin{array}{c}98.3 \\
(97.9- \\
98.5)\end{array}$ & $\begin{array}{c}98.1 \\
(98.0- \\
98.3)\end{array}$ & $\begin{array}{c}96.3 \neq \\
(96.0- \\
96.4)\end{array}$ & $\begin{array}{r}95.7 \neq \\
(95.3- \\
95.9)\end{array}$ & $\begin{array}{c}91.4 \dagger \\
(91.1- \\
91.8)\end{array}$ & $\begin{array}{c}91.1 \dagger \\
(91.0- \\
91.4)\end{array}$ \\
\hline Sensitivity (\%) & $\begin{array}{r}23.3 \neq \\
(21.6- \\
26.9)\end{array}$ & $\begin{array}{c}36.1 \neq \\
(34.0- \\
37.7)\end{array}$ & $\begin{array}{r}38.3 \neq \\
(37.5- \\
42.3)\end{array}$ & $\begin{array}{r}50.6 \neq \\
(48.4- \\
52.6)\end{array}$ & $\begin{array}{c}\mathbf{5 7 . 9} \\
(55.2- \\
59.8)\end{array}$ & $\begin{array}{r}64.5 \neq \\
(63.4- \\
66.2)\end{array}$ \\
\hline Precision (\%) & $\begin{array}{r}22.6 \neq \\
(18.8- \\
25.1)\end{array}$ & $\begin{array}{c}30.1 \neq \\
(27.5- \\
31.8)\end{array}$ & $\begin{array}{c}18.1 \neq \\
(16.5- \\
19.2)\end{array}$ & $\begin{array}{r}20.2 \neq \\
(18.3- \\
21.5)\end{array}$ & $\begin{array}{c}12.3 \neq \\
(12.0- \\
12.7)\end{array}$ & $\begin{array}{r}13.4 \neq \\
(12.9- \\
13.6)\end{array}$ \\
\hline MFDD (s) & $\begin{array}{l}104 \\
(91- \\
122)\end{array}$ & $\begin{array}{c}98 \\
(89- \\
103)\end{array}$ & $\begin{array}{r}114 \dagger \\
(109- \\
120)\end{array}$ & $\begin{array}{r}122+ \\
(112- \\
134)\end{array}$ & $\begin{array}{c}134 \\
(129- \\
137)\end{array}$ & $\begin{array}{r}133 \\
(131- \\
137)\end{array}$ \\
\hline Real FDR $\left(h^{-1}\right)$ & $\begin{array}{c}0.52 \\
(0.51- \\
0.53)\end{array}$ & $\begin{array}{c}0.53 \\
(0.51- \\
0.54)\end{array}$ & $\begin{array}{c}1.03 \\
(1.00- \\
1.04)\end{array}$ & $\begin{array}{c}1.03 \\
(1.01- \\
1.04)\end{array}$ & $\begin{array}{c}2.01 \\
(2.00- \\
2.06)\end{array}$ & $\begin{array}{c}2.02 \\
(1.99- \\
2.05)\end{array}$ \\
\hline
\end{tabular}

Table 4.1: Comparison for different false detection rates between metrics for algorithm performance without $(T)$ and with $\left(T^{+}\right)$quantification of temporal behaviour. FDR: false detection rate; GDR: good detection rate; MFDD: mean false detection duration. Values are given as median (minimum - maximum). Comparison between metrics of $T$ and $T^{+}$for each given FDR was conducted using the Wilcoxon signed ranks test. $\ddagger p<0.001 ;+p<0.01 ; * p<0.05$ 


\begin{tabular}{|c|c|c|c|c|c|c|}
\hline \multirow{2}{*}{$\begin{array}{l}\text { Set FDR }\left({ }^{-1}\right) \\
\text { Data set }\end{array}$} & \multicolumn{2}{|c|}{0.50} & \multicolumn{2}{|c|}{1.00} & \multicolumn{2}{|c|}{2.00} \\
\hline & $\mathrm{T}^{-}$ & $\mathrm{T}^{+}$ & $\mathrm{T}^{-}$ & $\mathrm{T}^{+}$ & $\mathrm{T}^{-}$ & $\mathrm{T}^{+}$ \\
\hline Category I (\%) & $\begin{array}{c}69.8+ \\
(61.7- \\
73.1)\end{array}$ & $\begin{array}{r}64.0+ \\
(58.9- \\
69.7)\end{array}$ & $\begin{array}{c}67.6 \\
(64.4- \\
70.6)\end{array}$ & $\begin{array}{c}65.8 \\
(60.0- \\
69.6)\end{array}$ & $\begin{array}{r}63.9+ \\
(62.2- \\
66.1)\end{array}$ & $\begin{array}{r}66.5+ \\
(63.4- \\
73.4)\end{array}$ \\
\hline Category II (\%) & $\begin{array}{c}\mathbf{2 7} . \mathbf{5}+ \\
(24.3- \\
35.7)\end{array}$ & $\begin{array}{c}33.2+ \\
(27.5- \\
38.3)\end{array}$ & $\begin{array}{c}29.5 \\
(26.4- \\
32.9)\end{array}$ & $\begin{array}{c}31.1 \\
(27.1- \\
37.0)\end{array}$ & $\begin{array}{r}33.0+ \\
(30.9- \\
34.6)\end{array}$ & $\begin{array}{r}30.2+ \\
(23.2- \\
33.4)\end{array}$ \\
\hline Category III (\%) & $\begin{array}{c}2.6 \neq \\
(2.6- \\
2.7)\end{array}$ & $\begin{array}{c}2.8 \neq \\
(2.6- \\
3.0)\end{array}$ & $\begin{array}{c}2.9 \neq \\
(2.8- \\
3.0)\end{array}$ & $\begin{array}{c}3.1 \neq \\
(3.0- \\
3.3)\end{array}$ & $\begin{array}{c}3.1 \dagger \\
(3.0- \\
3.3)\end{array}$ & $\begin{array}{c}3.3+ \\
(3.0- \\
3.7)\end{array}$ \\
\hline
\end{tabular}

Table 4.2: Comparison of distribution of undetected seizures in categories within the $T$ and $T^{+}$set for different false detection rates.

FDR: false detection rate; Category I: seizures with all epochs under detection threshold; Category II: seizures containing epochs above detection threshold, but less then the minimum number required for detection; Category III: seizures with partial overlap of recording start or end. Values are given as median (minimum - maximum). $\ddagger p<0.001$; $+p<0.01 ; * p<0.05$

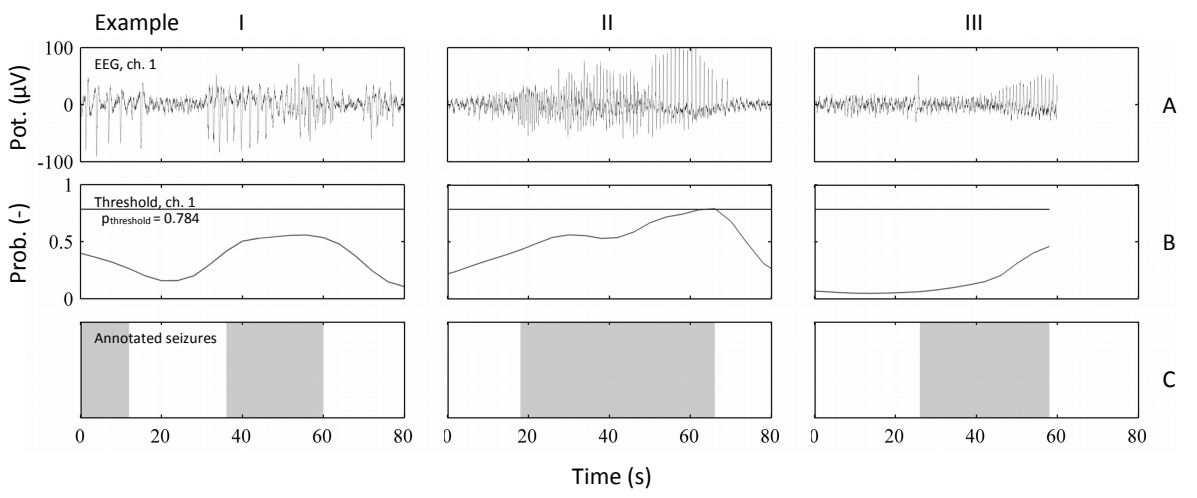

Figure 4.5: Three examples of undetected seizures. (A) EEG recording containing seizure activity. Note that the $y$-axis has been rescaled from Figure 4.3A to make seizure activity clearer. (B) Comparison of moving average filtered empirical probabilities (grey line) against the threshold. (C) Annotated seizure epochs (grey rectangle). The seizure in example I remained undetected because filtered empirical probabilities remained below the threshold. In example II filtered empirical probabilities exceed the threshold level, but the number of epochs where probabilities exceed threshold levels is below the required limit. The seizure in example III overlaps with the end of the recording and was not detected.

\section{Discussion}

GDR for short seizures increases significantly by quantifying temporal information through the use of trend templates for seizure onset and end. This occurs irrespective of the FDR at which performance is evaluated. Our findings indicate simultaneous 
increases in sensitivity and precision, and a small concomitant decrease in specificity. Trend templates significantly increase GDR as they capture information that is usually lost. Feature values change when transitioning from seizure to non-seizure state and vice-versa. Previous algorithms examine this change on a per-epoch level to differentiate between seizure and non-seizure activity, but lose all information contained in the behaviour of feature values during a sequence of epochs. It is exactly this information that we capture by using trend templates for seizure onset and end, and which demonstrably improves GDR.

Our GDR, even for short seizures, fell short of that achieved by Temko et al. ${ }^{49}$, who were able to correctly detect $72 \%$ of short seizures at 1.0 FDR. Although detection algorithm design is mostly comparable between us and Temko and colleagues, important differences exist between both studies which might explain the differences between the two studies. The most notable are the number of EEG channels available in recordings, and the number of SVM input features used for classification.

Though conventional eight-channel EEG is more sensitive to seizures ${ }^{114,115}$, interpretation and review of multi-channel EEG recordings is laborious and requires the presence of highly trained staff. Therefore, conventional EEG is not commonly used for long-term continuous monitoring purposes at the neonatal intensive care unit (NICU). Instead, one or two-channel amplitude-integrated EEG (aEEG) displayed together with continuous EEG is used because it involves less handling of the infant, is less obtrusive to the infant, and is relatively easy to interpret ${ }^{20,116}$. The results of our study apply to the common implementation of amplitude-integrated EEG monitoring in the NICU. One shortcoming of the aEEG monitor is that short seizures are difficult to assess ${ }^{115,117}$. Our algorithm could thus help overcome a major limitation of aEEG monitoring-detecting short seizures-without having to increase the number of EEG electrodes and risking an increase in patient discomfort.

One advantage of conventional eight-channel EEG over two-channel EEG with aEEG is the sensitivity to focal seizures ${ }^{118}$, but at the cost of increased obtrusiveness to the patient, increased patient handling and being complex to interpret. Seizure detection algorithms address the issue of increasing the time experts need for EEG evaluation, and could allow continuous EEG monitoring at the NICU, while at the same time increasing GDR compared to two-channel EEG. We consider the use of trend templates to be beneficial to detector performance in conventional EEG as well, though increases in performance are likely smaller than found in our study.

Detector performance might be further improved by increasing the number of EEG features used for SVM classification. Temko et al. used a feature set consisting of 55 features, not all of which need to be sensitive to seizures. Our set of features was chosen to be adequate for the purpose of testing our hypothesis. At the same time, it is probable that this choice limits detector performance because other features sensitive to seizures were not used. One advantage of using a support vector machine as classifier is its robustness to insensitive features ${ }^{119}$. Hence, introducing insensitive features is not inherently detrimental to detector performance, as long as not every feature is insensitive. Extending the feature set will allow the SVM classifier to more reliably classify data, leading to better detector performance. Increasing the number of features can be done in conjunction with our method of quantifying temporal changes 
in features by using trend templates. Features which are insensitive to seizures will introduce an average slope close to 0 in trend templates and thus will only negligibly contribute in the calculation of corresponding scalar resolutes.

More extensive and elaborate artefact suppression can reduce false detection rates $^{120}$. Though we suppressed high-amplitude and flat-line artefacts, it should be taken into account that EEG recordings are prone to many other artefacts. Some EEG artefacts resemble seizure activity in rhythmicity and amplitude, increasing the likelihood of incorrectly detecting artefacts as seizures. Notable examples of artefacts producing seizure-like signals are ECG-leakage-where dominant QRS-complexes causes rhythmic EEG spikes from 2 up to 4 times per second-and poorly contacting electrodes-causing rhythmic discharges in the EEG. Though other notable examples, such as rocking artefacts and subject handling by caretakers were not present in our fetal model, they can and do occur in neonates ${ }^{17}$, and could potentially introduce misdetections.

To assess the effect of using trend templates on detector performance for seizures shorter than one minute, we chose a transiently asphyxiated fetal sheep model. For an appropriate set of EEG recordings, two conditions are required, namely that it contains a large number of short seizures and that short seizures are distributed among subjects. The fetal sheep model fulfilled both conditions. As mentioned, the model is equivalent to the human preterm infant of 30-34 weeks of gestation. However, to estimate algorithm performance within the clinical setting of the NICU we would recommend using a set of EEGs recorded in human infants that represents the diverse degrees of maturation and disease etiologies encountered in practice.

To assess algorithm performance we used cross-validation. The standard approach to cross-validation only has two stages, classifier training and independent validation. Post-processing optimization has to be conducted on data assigned to either of these stages, which potentially introduces biases. To avoid this bias, we conducted crossvalidation using three stages: classifier training, post-processing optimization and independent validation. To our knowledge, this is the most rigorous approach to algorithm validation published so far for neonatal seizure detection algorithms-please refer to Temko et al. ${ }^{112}$ for a comparison with previous algorithms.

To conduct post-processing optimization, we defined an optimization target and two constraints. A commonly used optimization target is area under the curve (AUC) of the receiver operating characteristic. In this study there are two methodological drawbacks to AUC as an optimization target. The first drawback is the necessity of varying a single post-processing parameter while keeping other parameters constant when calculating the AUC. This approach does not produce a set of five fixed parameter values, which is required to analyse data in the independent validation fold. This problem could be handled by assessing AUCs over a complete parameter landscape, yet the resulting choice of parameters is still implicit. A second issue is that AUC is most useful in assessing classification performance on a per-epoch basis. Performance assessment which is most relevant to clinical practice is event-based, not epoch-based, and should assess the fraction of correctly detected seizures and the concomitant false detection rate. GDR can be calculated for any fixed combination of post-processing parameters. We used GDR as optimization target because GDR is both relevant and can be explicitly evaluated, instead of implicitly as is the case for AUC. 


\section{Limitations}

The main limitation of this study is the usage of preterm two-channel EEG recorded in sheep as input for classification by the algorithm. This setup mimics the two-channel EEG used for monitoring purposes in preterm and term infants. The animal model allowed us to specifically address the challenge of detecting short seizures $<1$ minute in duration. However two-channel EEG lacks the spatial resolution provided by the reduced 10-20 system, and the detection algorithm is likely to underperform when compared to algorithms from studies involving multi-channel recordings.

Another limitation is that we did not assess inter-rater reliability of seizure annotations and, hence, cannot state the degree of subjectivity induced by consensus scoring.

\section{Conclusion}

The use of trend templates for seizure onset and end in a neonatal seizure detection algorithm significantly improves the correct detection of short seizures ( $<1$ minute) using two-channel EEG recordings. This finding is relevant to both algorithm design and clinical application. Trend templates can be used in other neonatal seizure detection algorithms and are likely to improve detector performance. At the NICU short seizures are often missed during aEEG monitoring and our detection algorithm might aid in their detection. Before clinical application of the algorithm, we recommend that detector performance should be improved by increasing the number of input features and by artefact removal. Also, using multi-channel EEG is likely to improve detector performance, but at the risk of increasing patient discomfort. Furthermore the algorithm should be validated on human neonatal EEG recordings to better estimate performance within clinical settings. 



\section{Interhemispheric burst synchrony}

Published as:

Zwanenburg, A., Meijer E., Jennekens, W., Van Pul, C., Kramer, B. W. \& Andriessen, P. Automatic dectection of burst synchrony in preterm infants. Conf Proc IEEE Eng Med Biol Soc, 4720-4723 (2012) 



\section{Abstract}

Background Electroencephalographic characteristics are useful in assessment of the functional status of specific neuronal connections relative to postmenstrual age. Interhemispheric burst synchrony (IBS) is a measure of the functional connectivity between the hemispheres in the maturing preterm brain. Guidelines for observation of synchrony in the EEG are ambiguous, and may have led to different findings with regards to maturational effects in IBS.

Methods An algorithm was developed to assess IBS and was used in a longitudinal EEG study on 18 very preterm infants ( $<32$ weeks gestational age) with normal followup at 2 years of age. The preterm infants underwent weekly 4 -hour multi-channel EEG recordings, resulting in $n=77 \mathrm{EEGs}$. After automated detection of bursts, the algorithm defines the start and end of interhemispheric synchronous burst activity, based on selection criteria found in literature.

Results No significant changes in IBS occurred with the period of 28-36 weeks, and no significant maturational effect could be established.

Conclusion The computer algorithm may be used clinically to open novel avenues to automated analysis in EEG monitoring. Moreover, it may facilitate assessment of the functional status of interhemispheric connections. 


\section{Introduction}

Electroencephalography (EEG) is a very useful tool for predicting neurological outcome in preterm and term infants ${ }^{17,121,122}$. Interpretation of EEG, in clinical settings, is commonly performed by trained observers. However, inter-observer differences in interpretation and the time-consuming nature of visual inspection are drawbacks of the use of human observers, and the ongoing development of the preterm brain, resulting in EEG changes, imposes further difficulties. Interpretation of the EEG would, therefore, benefit from objective, automated analysis algorithms.

Several EEG patterns, such as bursts and interbursts, which are prevalent in the preterm $\mathrm{EEG}^{17}$, can display synchrony. Synchrony in the broad sense is the presence of similar EEG features of comparable duration measured almost simultaneously in different regions of the cortex. EEG synchrony is thought to reflect remote functional connectivity ${ }^{54}$. Interhemispheric burst synchrony (IBS) is synchrony between bursts in the left and right hemispheres. To measure IBS, both Lombroso and Anderson et al. visually inspected EEG recordings recorded during (quiet) sleep in otherwise healthy preterm neonates and compared the onset of bursts in both hemispheres ${ }^{56,59,123}$. Both groups studied changes in IBS with maturation. However they found conflicting results for the age group of 31 weeks conceptional age (CA) common to both studies. Whereas Lombroso found a large decrease in IBS compared to the previous week, Anderson found a non-significant difference. This is likely caused by the use of slightly different methods, as well as inter-observer differences. For instance, IBS is represented as a percentage, but the definition is not well-defined. Anderson et al. defined the percentage as the number of 1-minute epochs where bursts were synchronous divided by the total number of epochs ${ }^{59}$, whereas Lombroso defined it as the number of synchronous bursts as a fraction of the total number of bursts ${ }^{123}$. Moreover, what exactly qualifies as a synchronous burst differs between the authors, emphasizing the need for a clearer and more objective definition of IBS to facilitate comparison.

Furthermore, Tharp et al. demonstrated a close link between abnormal interhemispheric synchrony in preterm infants and death or major neurological sequela $\mathrm{e}^{55}$. However, because of the time-intensive nature of an IBS analysis, this measure is rarely used in clinical practice.

In this paper, we present a method for the automatic and objective detection of interhemispheric burst synchrony in otherwise healthy preterm neonates and we compare our results with those of Lombroso and Anderson et al.

\section{Methods}

Subjects

For this study 18 subjects were recruited from the neonatal intensive care unit. All subjects were preterm infants $<32$ weeks postmenstrual age (PMA, gestational age + postnatal age) at birth. The infants showed a normal follow-up at 2 years of age, with mental and motor scores $>85$ according to the international Bayley Scores of Infant Development ${ }^{124}$. Approval was given by the hospital ethics committee and written informed consent was given by the parents. For further details on the study group see 
Niemarkt et al. ${ }^{125}$. The infants underwent weekly 4-hour EEG recordings, starting in the second week after birth. The recordings $(n=77)$ represented a large variability in PMA (28- 36 weeks).

\section{Data aqcuisition and analysis}

Data acquisition Electrodes were placed according to the international reduced 10-20 montage system ${ }^{126}$. The signal was recorded with a common reference electrode. The signal was band-pass filtered between 0.30 and $35 \mathrm{~Hz}$ using a $4^{\text {th }}$ order forwardbackward Butterworth filter and sampled at sample frequency $f_{s}=256 \mathrm{~Hz}$. The recorded signal was recombined to form 13 channels: Fp1-Fp2, C3-C4, O1-O2, Fp1-C3, Fp1-T3, C3O1, C3-T3, O1-T3, Fp2-C4, Fp2-T4, C4-O2, C4-T4 and O2-T4.

Data processing Recordings were divided into non-overlapping 5 minutes segments. Artefacts were detected using a simple threshold technique and subsequently removed from analysis. The recording was subsequently analyzed using the algorithm described in this paper. Segments where artefacts were present for more than $5 \%$ of the duration and segments where continuous activity was present for more than $20 \%$ of the duration were excluded. This led to the complete exclusion of 3 EEG recordings.

IBS algorithm EEG recordings are analysed based on the algorithm developed by Jennekens et al. ${ }^{127}$. The algorithm generates a list by partitioning an EEG recording into burst, interburst, artefact, continuous and undefined sections. The algorithm defines bursts as activity with an amplitude $>30 \mu \mathrm{V}$, simultaneously occurring in $\geq 4$ channels and lasting $\geq 20 \mathrm{~s}$. Likewise, continuous activity was defined as bursts lasting $>20 \mathrm{~s}$. Defining IBS, we demand that the difference between different channels for both onset and end of the burst is within $1.5 \mathrm{~s}$, the duration of the burst section differs no more than $2.0 \mathrm{~s}$ between the channels $\mathrm{s}^{123}$ and that at least 4 channels are involved, including a pair of channels in mirror symmetrical locations on the two hemispheres, e.g. the pair C3-T3 and C4-T4. An example to illustrate the working of the algorithm is presented in Figure 5.1.

For each channel $b$ of the 13 channels we calculate the envelope function $E_{b, k}=\left(2 N_{w}^{-1} \sum_{i=k}^{k+N_{w}}\left(x_{b, i}\right)^{2}\right)^{1 / 2}$, where $k=1, \ldots, N-N_{w}$, with $N_{w}=256$ the number of samples over which the signal $\vec{x}_{b}$ is averaged and $N$ the number of samples in the recording. Then, for every burst we perform the following steps.

Step 1: The burst is, per channel, divided into sections where the envelope function exceeded the $30 \mu \mathrm{V}$ threshold value. The start and end times of all sections in all channels are calculated.

Step 2: Based on the start times from step 1, we find $L$ unique combinations of channels $\overrightarrow{f_{c, j}}$, with $j=1, \ldots, L$, where the difference in start times between the channels is less than 1.5 seconds. From this we derive the corresponding $L$ arrays $\overrightarrow{f_{t, j}}$ of start times. 


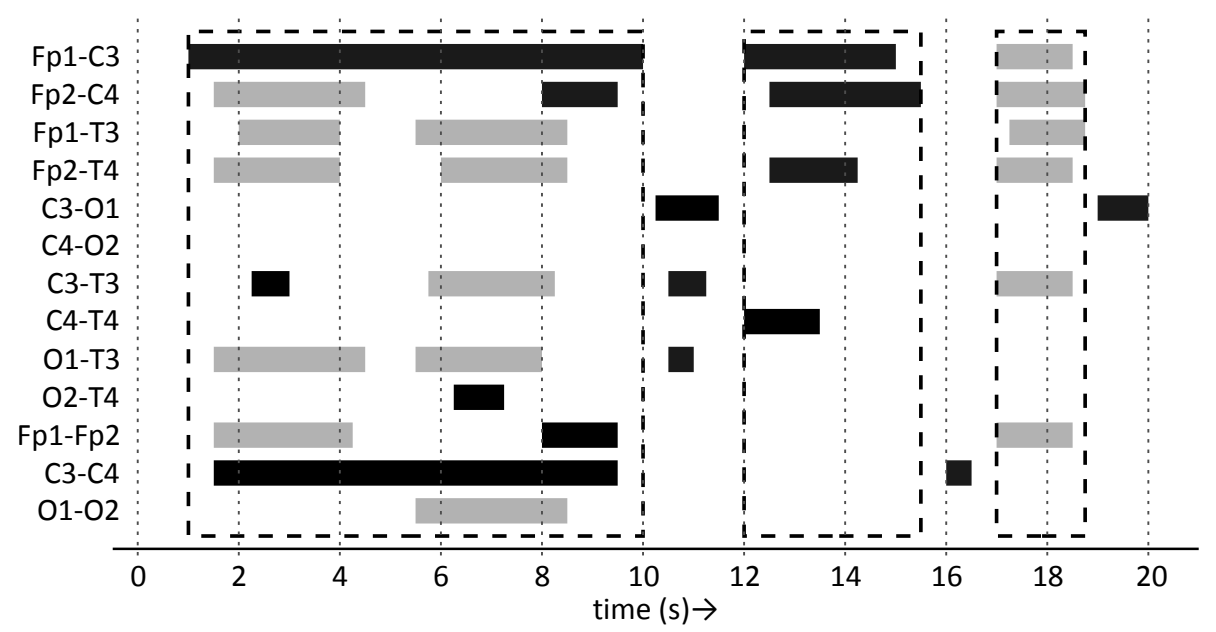

Figure 5.1: Example to illustrate the algorithm. For every channel, the sections where the activity exceeds the $>30 \mu \mathrm{V}$ threshold are shown as rectangles. The 3 bursts, as detected by the algorithm of Jennekens et al., are marked. Black rectangles represent sections that are not interhemispherically synchronous and light gray parts represent sections that are. The first burst actually consists of two separate synchronous bursts. The second burst is not interhemispherically synchronous, since the difference in end times and duration of the sections were too large.

Step 3: Step 2 is repeated for the end times of the sections found in step 1 . This yields $K$ unique combinations of channels $\overrightarrow{g_{c, k}}$, with $k=1, \ldots, K$ end time arrays $\overrightarrow{g_{t, k}}$.

Step 4: We generate all permutations of start and end times in the various channels. Having found $L$ channel combinations $\overrightarrow{f_{c, j}}$ and $K$ channel combinations $\overrightarrow{g_{c}, k}, K \cdot L$ channel combinations $\overrightarrow{h_{c, m}}=\overrightarrow{f_{c, j}} \circ \overrightarrow{g_{c, k}}$, where $\circ$ is the entrywise product operator and $m=1, \ldots, K \cdot L$, with corresponding start $h_{t, s, m}$ and end times $\overrightarrow{h_{t, e, m}}$, are formed.

Step 5: Most permutations contain invalid combinations of start and end times, e.g. when in a permutation there is a channel where the end of a burst section would precede the start thereof. The following checks are performed to remove invalid combinations: a: Remove all channels where the end time is before the start time. $\mathbf{b}$ : Remove all channels where the particular start time is not closest to the end time. c: Burst sections on different channels are demanded to be similar in duration, within 2 seconds. This creates one or more different combinations of channels, and we select the combination with the highest number of channels. If there is more than one of these, the combination of channels where the variance between durations is minimal is chosen.

Step 6: Subsequently, the interhemispheric synchrony of this selection is checked by demanding that at least one pair of mirror symmetrical channels in both hemispheres is involved. 
Step 7: If the combination of channels involves 4 or more channels, and was marked as synchronous in the previous step, $\overrightarrow{h_{c, m}}, \overrightarrow{h_{t, s, m}}$ and $\overrightarrow{h_{t, e, m}}$, corrected in step 5, are stored.

Step 8: After performing steps 5 to $7 K \cdot L$ times, $M_{p}$ combinations of channels $\overrightarrow{h_{c, k}}$, where $0 \leq M_{p} \leq K \cdot L$ and $k=1, \ldots, M_{p}$, with corresponding start and end times $h_{t, s, k}$ and $h_{t, e, k}$, are found that present interhemispheric synchronous bursts. Where $M_{p} \neq 0$, some of the combinations represent separate burst sections, while others are variations of each other. A separate burst section shares no start and end times on the involved channels with any other separate burst. An example of separate burst sections are shown in Figure 5.1. Within a burst section, it is possible that there are different combinations of channels that are essentially variations of each other, sharing at least one start or end time. We separate the various combinations into $M_{\text {sep }}$ separate sections, each section consisting of $M_{v, j}$ variations, where $j=1, \ldots, M_{\text {sep }}$.

Step 9: For each $j$ of the $M_{\text {sep }}$ burst sections we need to determine which of the $M_{v, j}$ variations is the most suitable, based on the temporal distribution of start and end times. To do this, we sum the variance in start times and the variance in end times for each $i$ of the $M_{v, j}$ variations and choose the variation $i$ for which the sum is minimal.

Thus, we have determined for each burst whether it is interhemispherically synchronous, and in case it is, the channels involved in the underlying burst sections, and their respective onset and end times, are stored.

\section{Statistics}

In general, IBS is a measure of the amount of interhemispheric synchronous bursts compared to the total amount of bursts. Unfortunately, IBS is calculated in several ways, based on subjective visual inspection of the $\mathrm{EEG}^{56,59}$. Therefore, to compare with the available literature we chose to use the following two representations of IBS, one based on the duration of synchronous bursts and another based on whether a burst can be marked as synchronous: 1) IBS 1 : The total duration of synchronous bursts-corrected for possible overlap between separate synchronous bursts on different channels-divided by the total duration of bursts. 2) IBS 2 : The number of bursts marked as synchronous divided by the total number of bursts, with the restriction that if a burst is a combination of separate synchronous bursts, only one is counted.

For each recording and each infant, the medians of the values for IBS 1 and IBS $S_{2}$ in the 5 -minute segments analyzed are calculated. From the medians, the population means are calculated and ranges (minimum value - maximum value) are given for different weeks PMA. Significance of changes for subsequent weeks is calculated using Student's two-sided paired $t$-test. A result with $p<0.05$ is considered significant.

Results found by Lombroso ${ }^{56}$ and Anderson et al..$^{59}$ are given in ranges, which were not further specified by the authors. 


\begin{tabular}{rrrrrrrrr} 
PMA (wks) & No. EEG & & IBS $_{1}(\%)$ & & IBS $_{2}(\%)$ & Lombroso (\%) & Anderson (\%) \\
\hline 28 & 3 & 56 & $(53-60)$ & 87 & $(83-88)$ & $90-100$ & \\
29 & 6 & 62 & $(58-69)$ & 86 & $(79-95)$ & $90-100$ & 92 & $(80-100)$ \\
30 & 13 & 59 & $(52-63)$ & 82 & $(74-91)$ & $90-100$ & & \\
31 & 12 & 58 & $(48-69)$ & 83 & $(71-89)$ & $80-100$ & 94 & $(79-100)$ \\
32 & 16 & 56 & $(47-70)$ & 83 & $(76-95)$ & $80-100$ & & \\
33 & 12 & 56 & $(44-65)$ & 85 & $(76-92)$ & $50-70$ & 94 & $(86-100)$ \\
34 & 7 & 56 & $(48-72)$ & 85 & $(79-94)$ & $50-70$ & & \\
35 & 5 & 51 & $(46-56)$ & 83 & $(76-88)$ & $60-80$ &
\end{tabular}

Table 5.1: In the first column the postmenstrual age (PMA) is given. The second column contains the number of recordings during the specific week. Third and fourth columns contain data for $I B S_{1}$ and $I B S_{2}$, given as mean and (min - max) range. The fifth column contains data from Lombroso ${ }^{56}$. The sixth column contains data from Anderson et al. ${ }^{59}$. Data in fifth and sixth columns were originally given as a function of conceptional age (CA) and converted to corresponding PMA for comparison.

\section{Results}

We analysed the available EEG recordings for our study group with the algorithm described above. Results are presented in Table 5.1 and in Figure 5.2.

Both representations of IBS show no significant changes between subsequent weeks, similar to the trend found by Anderson et al. Lombroso found a sharp decrease in the group of 33 weeks PMA and an increase afterwards. Values for IBS ${ }_{1}$ are lower than IBS 2 . Values for IBS ${ }_{1}$ are outside the ranges given by Lombroso except for 33 and 34 weeks PMA and are outside the range given by Anderson et al. altogether. Values for $\mathrm{IBS}_{2}$ partly overlap the ranges given by Lombroso except for 33 and 34 weeks PMA. These partly overlap the ranges given by Anderson et al. as well, though the values for $\mathrm{IBS}_{2}$ tend to be lower.

\section{Discussion}

A method to objectively characterize IBS in preterm infants was developed. Based on the partition of signals in burst and interburst patterns we were able to determine automatically, through several steps, the synchrony of a burst and the channels involved. The algorithm allows fast and automatic determination of IBS, making it feasible to use in hospital environments.

The algorithm was designed to closely follow the way one may visually inspect an EEG recording, as can be observed in Figure 5.1. We obtained criteria from literature to assess: 1) the correct channel combinations, 2) start and 3) end times of burst sections in the channels. In this way we were able to establish the interhemispheric synchrony of a single burst. 


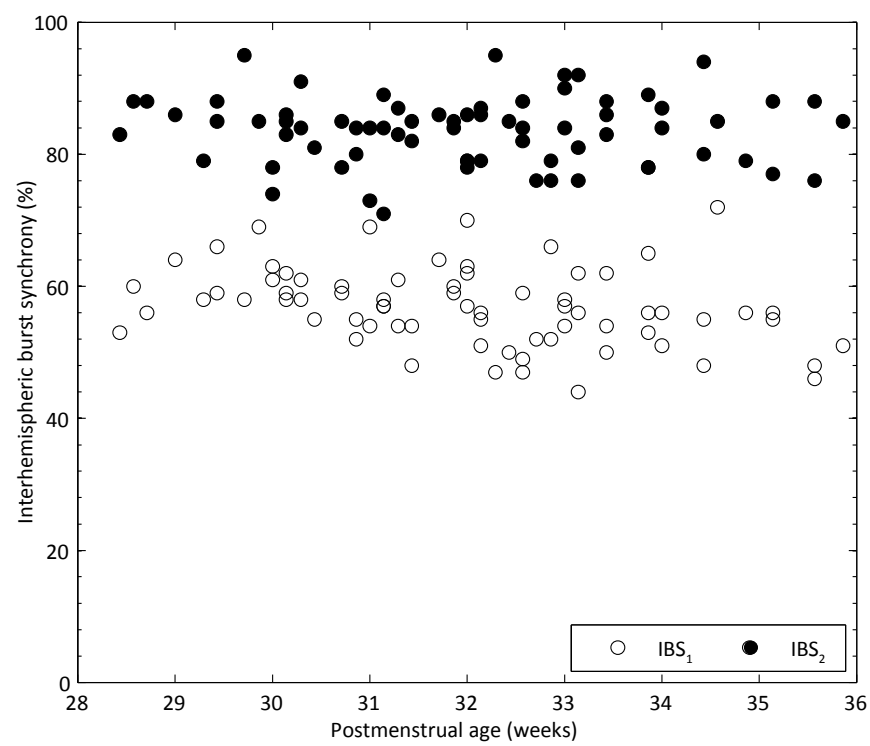

Figure 5.2: Interhemispheric burst synchrony as function of postmenstrual age for IBS 1 and IBS 2 . A data point represents the median value for the recording.

We were unable to find a clear definition of what constitutes an interhemispheric synchronous burst in literature. We restrict ourselves to synchronous bursts occuring in mirror symmetrical locations in both hemispheres, but it is possible that earlier research used a broader definition of bursts roughly simultaneously anywhere in both hemispheres, which is less strict. Our algorithm easily would accommodate a different definition by changing step 6 to look for burst sections on different channels anywhere, as long as they are in different hemispheres.

In our study, we found a difference between the two representations of IBS we proposed, as the range of values for IBS 1 was lower than for IBS 2 . We observed that this is caused by the definition of a burst as introduced by the burst detection algorithm used, which will extend a burst until the envelope function for all channels is below the threshold ${ }^{127}$. We observed that by removing this extension, values for IBS ${ }_{1}$ and IBS 2 became comparable, although ranges for $\mathrm{IBS}_{1}$ tended to remain lower.

Our data do not show the same trend as Lombroso: both IBS 1 and IBS 2 show no significant dependence on PMA. The data given by Lombroso are a composite of various studies, one of which is an earlier paper by Lombroso ${ }^{123}$, which describes the development of IBS from 33 to 43 weeks PMA. In this study, infants were recruited who were born between 32 and 34 weeks gestational age. In our study, as well as in Anderson et al., infants of varying gestational ages were included. We suggest that the different findings are partly caused by the wider range in gestational age at inclusion in our study, and the potential influence of processes related to the preterm birth. In our case, such processes are desynchronised, as within a certain week there are infants with different gestational ages at birth, whereas in the study of Lombroso, these influences would be mostly synchronised. We do not know if there is an influence of gestational 
age on IBS, if so, its not major. Whether EEG characteristics in general are significantly affected by the duration of extrauterine life in age-matched infants is uncertain, see e.g. Conde et al. ${ }^{128}$, Niemarkt et al. ${ }^{129}$, Scher et al. ${ }^{130}$ and Sisman et al. ${ }^{131}$. Another factor is that we did not look specifically at quiet sleep, due to lack of eye movement recordings, and resorted to excluding segments with continuous activity.

Though the data given by Anderson et al. are sparse, our results seem to follow the same trend. $\mathrm{IBS}_{2}$, which compares best to the results by Anderson et al., tends to be lower. This is due to different criteria for onset and end time differences between channels. We restricted this to $1.5 \mathrm{~s}$ maximum difference, in accordance with Lombroso, where Anderson et al. used $2.0 \mathrm{~s}$. Re-analysing the data with $2.0 \mathrm{~s}$ instead of $1.5 \mathrm{~s}$ resulted in higher values for $\mathrm{IBS}_{2}$, closer to the range found by Anderson et al, but without a change in trend.

\section{Conclusion}

The available literature shows no clear definition of burst synchrony. This study proposes a clear definition of IBS and, moreover, an objective way of determining synchrony. Further research should establish a range of values for IBS in normal preterm infants. Based on these ranges abnormal development of interhemispheric connectivity may be determined at an early stage, potentially indicating developing brain injury ${ }^{55}$. 


\section{Baroreceptor reflex function in HIE}

Adapted by rearrangement of sections from:

Zwanenburg, A., Jellema, R. K., Jennekens, W., Ophelders, D. R. M. G., Vullings, R., Van Hunnik, A., Van Pul, C., Bennet, L., Delhaas, T., Kramer, B. W. \& Andriessen, P. Heart rate mediated blood pressure control in preterm fetal sheep under normal and hypoxicischemic conditions. Ped Res 73, 420-426 (2013) 



\section{Abstract}

Background The understanding of hypoxemia-induced changes in baroreflex function is limited and may be studied in a fetal sheep experiment before, during, and after standardized hypoxic conditions.

Methods Preterm fetal lambs were instrumented at 102 days gestation (term: 146 days). At 106 days intra-uterine hypoxic-ischemia was induced by 25 -min of umbilical cord occlusion (UCO). Baroreflex related fluctuations were calculated at 30-min intervals during the first week after UCO by transfer function (cross-spectral) analysis between systolic blood pressure and R-R interval fluctuations, estimated in the low frequency (LF, 0.04-0.15 Hz) band. LF transfer gain (baroreflex sensitivity) and delay (s) reflect the baroreflex function.

Results Baseline showed no differences in LF transfer gain and delay between controls and UCO. In controls, LF gain showed postnatal increase. In contrast, LF gain gradually decreased in the UCO group, resulting in significantly lower values 4-7 days after UCO. In the UCO group, LF delay increased and differed significantly from controls.

Conclusion Our results show that intra-uterine hypoxic-ischemia results in reduced baroreflex sensitivity over a period of 7 days, indicating limited efficacy to buffer blood pressure changes by adapting heart rate. Cardiovascular dysregulation may augment already present cerebral damage after systemic hypoxic-ischemia in the reperfusion period. 


\section{Introduction}

Preterm infants have a higher incidence of neurological morbidity and mortality compared to term infants ${ }^{132}$. Neurological sequelae in preterm infants may be the consequence of hypoxic-ischemia during fetal and early postnatal life ${ }^{133}$. Hemodynamic factors during hypoxic-ischemia and the post-hypoxic-ischemia reperfusion phase contribute considerably to these neurological disorders ${ }^{134}$. To ensure adequate blood flow to organs throughout the body, including the brain, blood pressure (BP) is controlled by baro- and chemoreflex mechanisms. Under normoxaemia, the baroreflex stabilizes perfusion pressure in the face of disturbances of circulatory homeostasis by adapting heart rate, myocardial contraction and vascular resistance. A poorly developed baroreflex function could contribute to blood pressure instabilities, which may lead to impaired cerebral perfusion or hemorrhage $\mathrm{e}^{135}$. A better understanding of the dynamics underlying the control mechanisms regulating blood pressure may be useful to improve diagnosis of these disorders.

Low-frequency (LF) fluctuations in arterial blood pressure with a wave length of approximate $10 \mathrm{~s}$ (also called Mayer waves) have historically been attributed to baroreflex activity ${ }^{136}$. The oscillations are assumed to be caused by a feedback control and mediated through the sympathetic and parasympathetic innervations of the baroreflex ${ }^{137}$. Assessment of the baroreflex function (i.e. heart rate mediated blood pressure control) can be obtained through quantification of baroreceptor sensitivity. Baroreceptor sensitivity $(\mathrm{ms} / \mathrm{mmHg}$ ) may simply be defined as the change in heart rate (or R-R interval) in response to changes in arterial blood pressure, and may be estimated from LF cross spectral analysis of spontaneous fluctuations in R-R intervals and blood pressure ${ }^{138-141}$.

Our understanding of the cardiovascular response of the human preterm infant is limited ${ }^{142,143}$ and knowledge of fetal baroreflex is mostly based on animal (sheep) models ${ }^{25,26}$. There is, however, conflicting evidence on the fetal and postnatal development of baroreceptor reflex function. While some studies show increasing baroreflex sensitivity with age, other studies show no significant maturational change ${ }^{144-147}$. The cardiovascular system responds to hypoxic-ischemia by changing heart rate and redistributing cardiac output to preserve the limited energy resources and maintain adequate supply to the brain. Current knowledge about how the preterm fetus, hampered by an immature blood pressure regulation, responds to hypoxia is limited. Experiments have shown that the immature sheep fetus can survive prolonged periods of hypoxic-ischemia, but resulting hypotension and hypoperfusion may contribute to cerebral injury as seen in human preterm fetuses ${ }^{25,87,148}$. These studies mainly focus on cerebrovascular and fetal heart rate response following hypoxicischemia for $72 \mathrm{~h}$. Heart rate mediated blood pressure control has not been examined for longer than several days after the initial period of hypoxic-ischemia.

The aim of this study was (a) to quantify baroreflex mediated heart rate response (baroreflex sensitivity) by transfer function analysis between low-frequency $R-R$ and systolic blood pressure fluctuations in an instrumented preterm fetal sheep model and (b) to evaluate longitudinal changes of baroreflex mediated heart rate response during the first week following umbilical cord occlusion (UCO) induced hypoxic-ischemia. 


\section{Methods}

\section{Animal experiment}

Animals Texel ewes were date-mated and the fetuses were randomized for UCO or control. The study group consisted of 14 fetal sheep (9 controls and 5 UCO). Fetal instrumentation was performed at 0.7 GA (102 days, term 146 days). The study was conducted at Maastricht University Medical Center, Maastricht, The Netherlands, and was approved by the local Animal Ethics Research Committee.

Experimental preparations The pregnant ewes were deprived of food, but not water, $24 \mathrm{~h}$ before surgery. They were anesthetized using 1-2\% isoflurane, guided by depth of sedation. After a lower midline incision, the fetus was carefully extracted through a small incision of the uterus. The fetus was instrumented with a 3.5 French Argyle umbilical vessel catheter (Tyco Health Care Group, Mansfield, MA) in the femoral artery. The arterial catheter was utilized for continuous monitoring of fetal arterial pressure. A vascular occluder (OC16HD, in vivo Metric, Healdsburg, CA ) was placed around the umbilical cord. Three custom-made silver electrodes with fixed leads (Cooner, Chatsworth, CA) were inserted in the subdermis and fixed with sutures. These electrodes were used for electrocardiography and formed an Einthoven triangle. The fetus was then repositioned in the uterus. A catheter was inserted into the amniotic sac to monitor the amniotic fluid pressure. Before closure the amniotic cavity was filled with warm sterile saline ( $\mathrm{NaCl} 0.9 \%)$. All fetal catheters and electrode leads were exteriorized through the flank of the ewe. The lower midline incision was securely sutured. After surgery, the ewe was allowed to recover and housed in a confined space to allow continuous perfusion of catheters with heparinized saline. Post-surgery ewes received five days of intravenous amoxicillin (1000 mg) / clavulanic-acid (200 mg). During the complete course of the experiment ( 11 days) the animals had ad libitum access to water and food.

Experimental design Experiments were conducted at 102 (operation) to 113 (sacrifice) days of gestation. After a recovery period of four days (GA, 106 days) the umbilical cord of the fetuses in the UCO group was occluded by rapidly inflating the occluder with sterile saline of a defined volume known to completely inflate the occluder. Verification of the occlusion was obtained by observation of a sudden drop in heart rate. The occlusion was discontinued after $25 \mathrm{~min}$, or earlier when mean arterial blood pressure dropped below $8 \mathrm{mmHg}$ or asystole occurred. At the end of the occlusion, the lambs always suffered from severe bradycardia (heart rate $<90 \mathrm{bpm}$, normal approximately $180 \mathrm{bpm}$ ) or even complete cardiac arrest. If there was no increase in heart rate within $20 \mathrm{~s}$ after the end of occlusion, resuscitation was started using an injection of adrenalin $(0.1 \mathrm{mg} / \mathrm{kg})$. Arterial blood gas analysis $\left(\mathrm{PaO}_{2}, \mathrm{PaCO}_{2}, \mathrm{pH}\right)$ was performed before, during and after umbilical cord occlusion to monitor the metabolic state during hypoxicischemia and recovery. Fetuses were studied for 7 days post-occlusion. At the end of the experiment, both ewe and fetus were euthanized by administration of pentobarbital (200 mg/kg). 


\section{Data acquisition and analysis}

Data acquisition Blood pressure, amniotic pressure and ECG data were acquired and digitized by an MPAQ unit (Maastricht-Programmable AcQuisition system, Maastricht Instruments BV, Maastricht, The Netherlands). Analog filtering was applied to the ECG data, with a 1-200 $\mathrm{Hz}$ band-pass filter. Arterial blood pressure and amniotic pressure data were not filtered. All data were sampled at $1000 \mathrm{~Hz}$. Finally, data were stored on a hard drive for off-line analysis.

Data analysis Data were processed off-line using MATLAB R2009a (The MathWorks, Inc., Natick, MA). Recordings were divided into 30-min segments for analysis. ECG data were filtered using a $50 \mathrm{~Hz}$ infinite impulse response notch filter and $20-80 \mathrm{~Hz}$ 4th-order Butterworth band-pass filter, empirically found to be optimal for the R-peak detection algorithm used. Fetal blood pressure signals were filtered using a $50 \mathrm{~Hz}$ infinite impulse response notch filter and 2-16 Hz 4th-order Butterworth band-pass filter to facilitate detection by the waveform analysis algorithm. Fetal blood pressure was corrected for offset by subtraction of the low-pass filtered amniotic fluid pressure.

In each 30-min segment, R-peaks of the QRS-complex and systolic blood pressure (SBP) were detected by adaptive filtering of the ECG and blood pressure waveforms, respectively. Segments containing artifacts were excluded ( $<5 \%$ of the data). The unevenly time-spaced R-R interval and SBP series were convolved with a boxcar function and subsequently resampled at $4 \mathrm{~Hz}$ using nearest-neighbor interpolation to obtain equidistant sample points.

\section{Spectral analysis and transfer-function analysis Spectral analysis offers the} opportunity to decompose spontaneously occurring fluctuations in SBP and R-R interval time series into a power spectrum, and to relate the character of the fluctuations to physiological processes. Transfer function analysis (cross-spectral analysis) between SBP and R-R interval series in the low-frequency band $(0.04-0.15 \mathrm{~Hz})$ is a useful estimate of baroreflex sensitivity ${ }^{138,149,150}$. This methodology was adapted for preterm infants and we refer to previous papers for further details. As R-R interval and blood pressure values obtained from fetal sheep are comparable with values found in preterm infants, the same spectral analysis methodology was followed. Each 30-min segment of R-R interval and SBP series was divided into nine 192-s segments. In the 192-s segments total spectral power $(0.04-2 \mathrm{~Hz})$ and LF $(0.04-0.15 \mathrm{~Hz})$ spectral power were calculated from the auto spectra of $\mathrm{R}-\mathrm{R}$ interval and SBP series. From the cross spectra of $\mathrm{R}-\mathrm{R}$ interval and SBP series, the coherence, transfer gain and transfer phase were calculated for the LF band. As the reliability of transfer function depends on coherence between the signals, transfer function parameters were only assessed if coherence $>0.50$. In $41 \%$ of all 192-s segments coherence was $>0.50$ and these segments were included for transfer function analysis. Baroreflex sensitivity was calculated as the LF transfer gain and assessed at the frequency with the highest coherence within the LF band. Baroreflex delay was calculated by dividing the transfer phase at the corresponding frequency bin by $2 \pi$-times this frequency. Time domain cross correlation of the R-R interval and SBP series was used to correct for $n \cdot 2 \pi$ phase shifts.

Time domain estimates of variability Heart rate variability was assessed for each 192$\mathrm{s}$ segment by calculating the mean R-R interval and standard deviation (SD-RR) ${ }^{80}$. Likewise, blood pressure variability was assessed by calculating the mean SBP and 
standard deviation (SD-SBP). To verify results obtained by spectral analysis, baroreceptor sensitivity was also analyzed in the time domain. To obtain a time domain estimate for baroreceptor sensitivity, SD-RR was divided by SD-SBP ${ }^{150,151}$.

Data processing Median values per 30-min segment were calculated from the underlying 192-s segments. For further analysis, 30-min segments were pooled as $6 \mathrm{~h}$ or $24 \mathrm{~h}$ values (median; IQR), depending on the mode of presentation in graph or table, respectively. The median was used because normality was not $a$ priori assumed and to reduce the effect of outliers. Baseline values were assessed in the $24 \mathrm{~h}$ before UCO and median values for the long-term follow-up were assessed between 0-24, 24-48, 48-72, 72-96, 96-120, 120-144 and 144-168 h after UCO.

\section{Statistics}

Blood gas data were expressed as mean \pm SEM. Group comparisons for blood gas analysis were performed using the two-sided unpaired student $t$-test. Time and frequency domain estimates were expressed as median and IQR and group comparisons were analyzed using the Mann-Whitney $U$ test. As the 6-h values were normal distributed, the maturational effects on time domain and frequency domain spectral measures were evaluated using a multilevel analysis. In this analysis linear regression is estimated using the random effects model (SPSS 19.0.0, Inc., Chicago, IL), which extends the generalized linear model to allow for analysis of repeated 6 - $h$ values between and within subjects. Regression analysis results are shown with coefficients $a$ and $b(a$, intercept; $b$, slope of regression line). $p<0.05$ was considered significant.

\section{Results}

Cardiovascular and transfer function parameters at baseline Baseline values for both the UCO and the control group were derived in the $24 \mathrm{~h}$ before the UCO (Table 6.1). Time domain estimates of variability were comparable in both groups. A slight but significant difference in coherence values was noted between both groups. The baseline values for LF transfer gain, delay and frequency were comparable in both groups.

Cardiovascular and transfer function parameters at 144-168 $\mathrm{h}$ At the end of the experiment, mean R-R interval, mean systolic blood pressure and SBP standard deviation showed no differences between the intervention group and controls (Table 6.1). In contrast, the R-R interval standard deviation (SD-RR) was significantly reduced after UCO at comparable endpoint. Spectral power analysis showed significant differences in LF and total power for the R-R interval and SBP series between UCO and controls. In the UCO group, LF and total power for the R-R interval showed a 15 to 20 times reduction, compared to controls. For the SBP series, LF and total power were reduced by a factor of 2 . Transfer function analysis showed no difference in coherence or LF frequency. A slight increase in LF delay after UCO was observed compared to controls. Following UCO, both time domain estimate for baroreflex sensitivity (SDRR/SD-SBP) and transfer gain were reduced by approximately $40 \%$.

Maturation of cardiovascular and transfer function parameters Figure 6.1 shows the changes in short-term variability of R-R interval time series (SD-RR) and SBP (SD-SBP) time series, respectively. After UCO the decrease in SD-RR is reflected by decrease from 
72-168 $\mathrm{h}$ in SD-RR/SD-SBP, time domain estimate for baroreflex sensitivity. Figure 6.2 shows the changes in coherence, LF transfer gain and delay. From $96 \mathrm{~h}$, LF transfer gain is decreased versus controls.

In addition, the maturational effects on time and frequency domain spectral measures were evaluated by multilevel regression analysis for both the control and UCO group using pooled 6-hr values during the first week after UCO. This model corrects for the repeated measurements within and between subjects. The results of the multilevel analysis are shown in Table 6.2. The control group showed a significant increase in R-R interval, SD-RR, SBP and SD-SBP. The UCO group showed a significant increase in SD-SBP, approximately 2.5 -fold the magnitude of the SD-SBP increase in the control group. The multilevel analysis supported the finding that baroreflex sensitivity increases in controls, but decreases gradually after UCO induced asphyxia. Both time domain estimate for baroreflex sensitivity (SD-RR/SD-SBP) and LF transfer gain decreased by the same amount following UCO, $-0.7 \mathrm{~ms} / \mathrm{mmHg} /$ day. 
Control

UCO

\begin{tabular}{|c|c|c|c|c|}
\hline & Baseline & $\mathrm{T}=144-168 \mathrm{~h}$ & Baseline & $\mathrm{T}=144-168 \mathrm{~h}$ \\
\hline \multicolumn{5}{|l|}{ Blood gas analysis } \\
\hline $\mathrm{pH}$ & $7.41 \pm 0.04$ & $7.38 \pm 0.05$ & $7.41 \pm 0.03$ & $7.40 \pm 0.07$ \\
\hline Arterial $\mathrm{pCO}_{2}(\mathrm{mmHg})$ & $42 \pm 6$ & $31 \pm 10$ & $41 \pm 9$ & $41 \pm 10$ \\
\hline Arterial $\mathrm{pO}_{2}(\mathrm{mmHg})$ & $17 \pm 2$ & $17 \pm 3$ & $15 \pm 4$ & $19 \pm 6$ \\
\hline \multicolumn{5}{|l|}{$\mathbf{R}-\mathbf{R}$ interval series } \\
\hline $\mathrm{R}-\mathrm{R}$ interval (ms) & $\begin{array}{r}328 \\
(315-341)\end{array}$ & $\begin{array}{r}345 \\
(327-363)\end{array}$ & $\begin{array}{r}332 \\
(322-341)\end{array}$ & $\begin{array}{r}347 \\
(332-357)\end{array}$ \\
\hline SD-RR (ms) & $\begin{array}{r}21 \\
(15-25)\end{array}$ & $\begin{array}{r}21 \\
(18-27)\end{array}$ & $\begin{array}{r}21 \\
(17-24)\end{array}$ & $\begin{array}{r}11^{*} \\
(9-16)\end{array}$ \\
\hline Total power $\left(\mathrm{ms}^{2}\right)$ & $\begin{array}{r}184 \\
(54-451)\end{array}$ & $\begin{array}{r}271 \\
(149-463)\end{array}$ & $\begin{array}{r}258 \\
(46-406)\end{array}$ & $\begin{array}{r}13^{\star} \\
(2-71)\end{array}$ \\
\hline LF power $\left(\mathrm{ms}^{2}\right)$ & $\begin{array}{r}138 \\
(44-144)\end{array}$ & $\begin{array}{r}214 \\
(144-335)\end{array}$ & $\begin{array}{r}116 \\
(37-359)\end{array}$ & $\begin{array}{r}13^{*} \\
(1-62)\end{array}$ \\
\hline \multicolumn{5}{|l|}{ SBP series } \\
\hline $\mathrm{SBP}(\mathrm{mmHg})$ & $\begin{array}{r}46 \\
(43-48)\end{array}$ & $\begin{array}{r}49 \\
(45-50)\end{array}$ & $\begin{array}{r}47 \\
(39-55)\end{array}$ & $\begin{array}{r}52 \\
(45-57)\end{array}$ \\
\hline SD-SBP (mmHg) & $\begin{array}{r}1.8 \\
(1.7-2.1)\end{array}$ & $\begin{array}{r}2.2 \\
(2.0-2.3)\end{array}$ & $\begin{array}{r}1.8 \\
(1.7-1.9)\end{array}$ & $\begin{array}{r}1.9 \\
(1.7-2.4)\end{array}$ \\
\hline Total power $\left(10^{-2} \mathrm{mmHg}^{2}\right)$ & $\begin{array}{r}1.7 \\
(1.2-2.7)\end{array}$ & $\begin{array}{r}3.2 \\
(2.1-4.1)\end{array}$ & $\begin{array}{r}1.7 \\
(1.0-2.0)\end{array}$ & $\begin{array}{r}1.3 \dagger \\
(0.7-2.2)\end{array}$ \\
\hline LF power $\left(10^{-2} \mathrm{mmHg}^{2}\right)$ & $\begin{array}{r}1.5 \\
(1.1-2.5)^{-}\end{array}$ & $\begin{array}{r}2.4 \\
(1.9-3.5)\end{array}$ & $\begin{array}{r}1.6 \\
(0.9-1.8)\end{array}$ & $\begin{array}{r}1.3 \dagger \\
(0.6-2.2)\end{array}$ \\
\hline SD-RR/SD-SBP (ms/mmHg) & $\begin{array}{r}10.4 \\
(8.6-11.9)\end{array}$ & $\begin{array}{r}10.5 \\
(8.7-12.0)\end{array}$ & $\begin{array}{r}12.2 \\
(8 \cdot 6-13 \cdot 2)\end{array}$ & $\begin{array}{r}6.2^{\star} \\
(3.4-8.0)\end{array}$ \\
\hline \multicolumn{5}{|l|}{ Transfer function } \\
\hline Frequency $(\mathrm{Hz})$ & $\begin{array}{r}0.09 \\
(0.09-0.10)\end{array}$ & $\begin{array}{r}0.09 \\
(0.09-0.11)\end{array}$ & $\begin{array}{r}0.09 \\
(0.09-0.11)\end{array}$ & $\begin{array}{r}0.09 \\
(0.08-0.09)\end{array}$ \\
\hline Coherence & $\begin{array}{r}0.62 \\
(0.61-0.65)\end{array}$ & $\begin{array}{r}0.65 \\
(0.63-0.68)\end{array}$ & $\begin{array}{r}0.64 \dagger \\
(0.63-0.67)\end{array}$ & $\begin{array}{r}0.63 \\
(0.61-0.65)\end{array}$ \\
\hline Gain (ms/mmHg) & $\begin{array}{r}6.2 \\
(5.6-8.4)\end{array}$ & $\begin{array}{r}7.7 \\
(6.7-10.6)\end{array}$ & $\begin{array}{r}7.8 \\
(5.6-11.4)\end{array}$ & $\begin{array}{r}4.8^{*} \\
(3.1-6.7)\end{array}$ \\
\hline Delay (s) & $\begin{array}{r}5.5 \\
(3.3-6.8)\end{array}$ & $\begin{array}{r}4.3 \\
(3.2-5.9)\end{array}$ & $\begin{array}{r}4.0 \\
(3.3-5.6)\end{array}$ & $\begin{array}{r}6.6+ \\
(3.7-7.6)\end{array}$ \\
\hline
\end{tabular}

Table 6.1: Blood gas analyses, cardiovascular parameters and transfer function parameters during the baseline period and on the seventh day ( $T=144-168 \mathrm{~h})$ after UCO, respectively. Time domain and transfer function measures were determined from 30min segments during 24 hours before and 144-168 h after occlusion, respectively. Values are expressed as mean \pm SEM or median (IQR). ${ }^{*} p<0.01 ;^{\dagger} p<0.05$ (comparison between UCO and control at corresponding time). 
Baseline

UCO

$b$ (change/day)

$b$ (change/day)

\section{R-R interval series}

$\mathrm{R}-\mathrm{R}$ interval (ms)

$2.8 \pm 0.6^{*}$

NS

SD-RR (ms)

$0.8 \pm 0.2 *$

NS

SBP series

$\mathrm{SBP}(\mathrm{mmHg})$

$0.4 \pm 0.1 *$

NS

SD-SBP ( $\mathrm{mmHg}$ )

$0.04 \pm 0.02 \dagger$

NS

$0.10 \pm 0.02 *$

SD-RR/SD-SBP ( $\mathrm{ms} / \mathrm{mmHg}$ )

(1)

$-0.7 \pm 0.1 *$

Transfer function

Frequency $(\mathrm{Hz})$

NS

NS

Coherence

NS

NS

$\mathrm{LF}$ gain $(\mathrm{ms} / \mathrm{mmHg}$ )

LF delay (s)

$0.4 \pm 0.1 *$

$-0.7 \pm 0.1^{*}$

NS

$0.5 \pm 0.1 *$

Table 6.2: Results of multilevel regression analysis using a generalized linear model to correct for repeated measurements. Regression analysis is performed according to:

$f(t)=a+b \cdot t$, with $a$ the offset, $b$ the slope and $t$ the time (days) with $t=0$ corresponding to the time of occlusion for the UCO group. Values are expressed as mean $\pm S D$. * $p<0.01 ;+p<0.05$. 


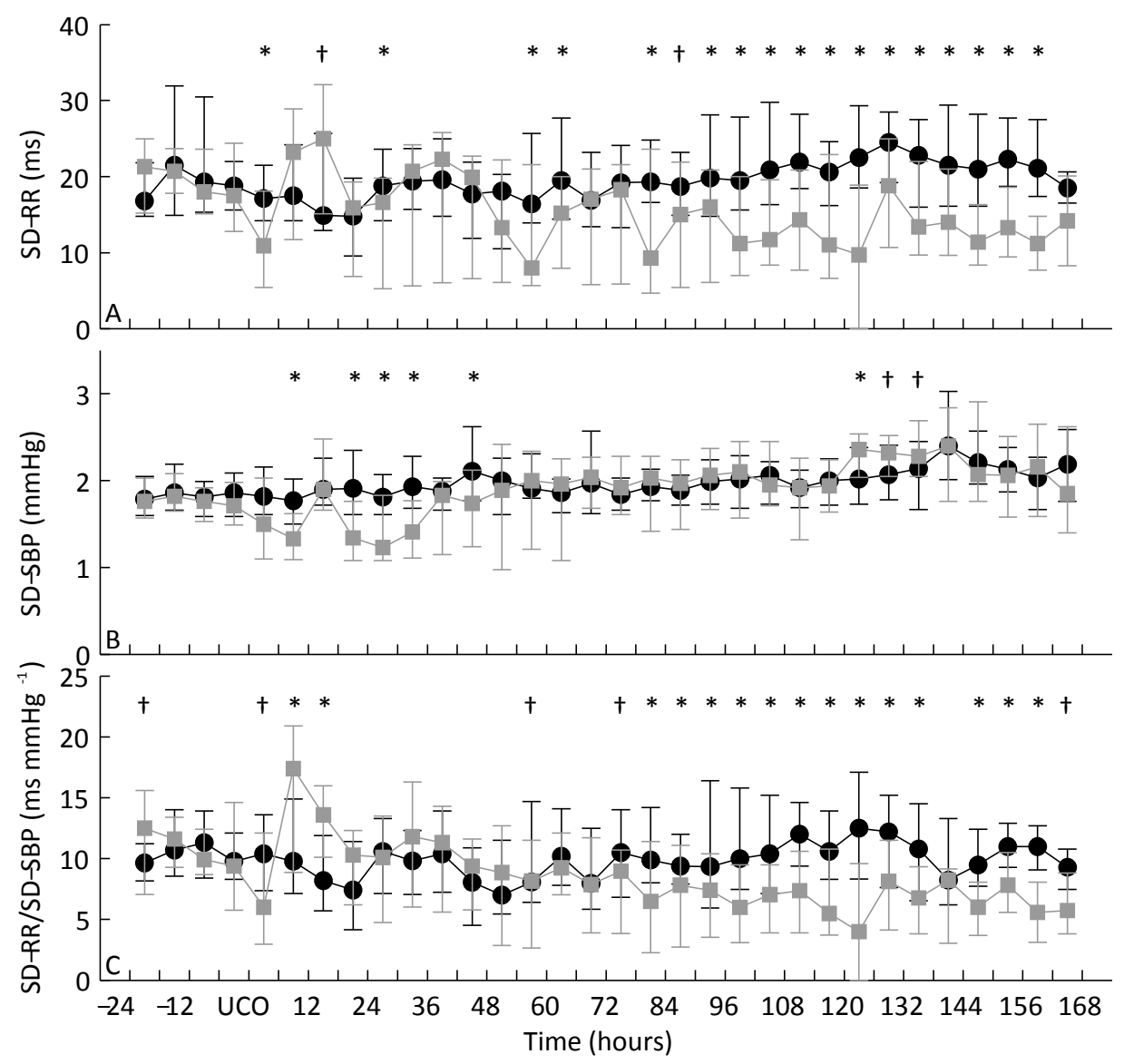

Figure 6.1: Longitudinal changes in SD-RR, SD-SBP and SD-RR/SD-SBP.Legend: Plots of the longitudinal development of heart rate variability $(S D-R R, A)$, blood pressure variability $(S D-S B P, B)$ and time domain measure of baroreflex sensitivity (SD-RR/SD-SBP, $C)$. The $x$-axis represents time in $h$. The black and gray symbols indicate the control and UCO group, respectively. The first $24 \mathrm{~h}$ after UCO significant differences are observed between UCO and control group, inherent to the experimental occlusion. After $72 \mathrm{~h}$ a consistent decrease in SD-RR and SD-RR/SD-SBP is noted. Data are shown as median and IQR for each 6- $h$ period. * $p<0.01 ;+p<0.05$ 


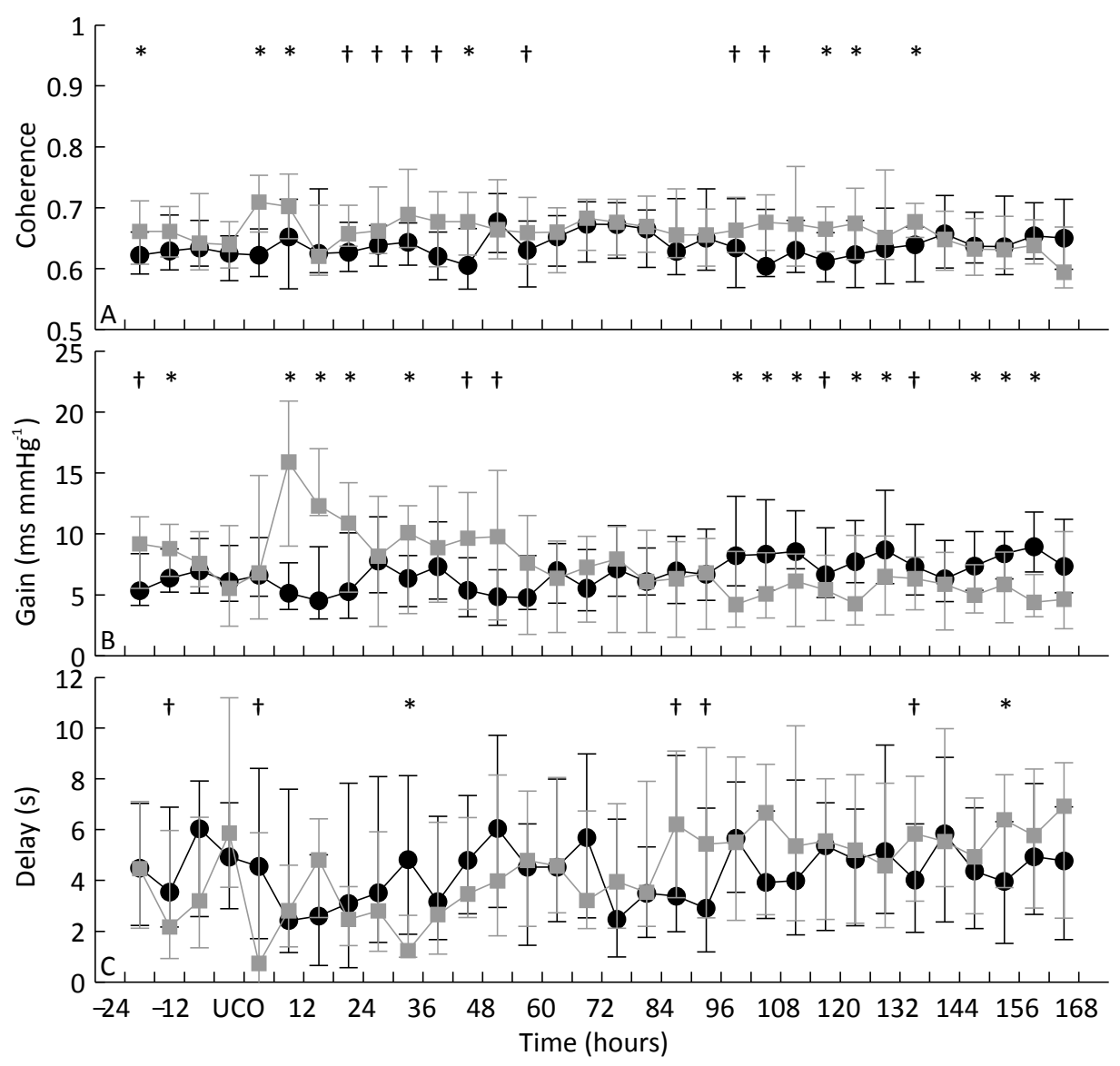

Figure 6.2: Longitudinal changes in coherence, LF transfer gain and delay. Legend: Plots of the longitudinal development of coherence $(\boldsymbol{A})$, transfer gain $(\boldsymbol{B})$ and delay $(\boldsymbol{C})$. The $\mathrm{X}$ axis represents time in $h$. The black and gray symbols indicate the control and UCO group, respectively. The first $24 \mathrm{~h}$ after UCO significant differences are observed between UCO and control group, inherent to the experimental occlusion. After $96 \mathrm{~h}$ a consistent decrease in LF transfer gain is noted. Data are shown as median and IQR for each 6-h period. * $p<0.01 ;+p<0.05$

\section{Discussion}

The present study demonstrates that intra-uterine induced hypoxic-ischemia in preterm fetal sheep has long-term effects on baroreflex function. During the first week following prolonged umbilical cord occlusion, heart rate variability decreases dramatically and baroreflex sensitivity decreases gradually to show lower values than controls at 4-7 days after UCO. These results suggest that intra-uterine hypoxic-ischemia limits the baroreflex activity to buffer changes in SBP by adapting fetal R-R interval. The dysregulation of the baroreflex may augment already present cerebral damage after systemic hypoxic-ischemia in the reperfusion period. As a consequence of this cardiovascular instability the preterm brain may be prone to blood pressure 
fluctuations, leading to disruption of the immature vascular architecture and neonatal encephalopathy ${ }^{62}$.

Transfer function analysis and baroreflex function In the present study we focused on the baroreflex-mediated interactions between LF fluctuations of SBP and R-R intervals during the normoxaemic recovery state, hence after the immediate cardiovascular effects of acute hypoxia and hypoxic-ischemia. Under normoxaemic condition, baroreflex rather than chemoreflex is involved in the interactions between arterial blood pressure and fetal heart rate ${ }^{152}$. We were interested in the long-term effect of induced fetal hypoxic-ischemia on baroreflex function, i.e. baroreflex sensitivity and delay of the reflex arc. The evaluation of baroreflex function is useful for the assessment of fetal autonomic cardiovascular control ${ }^{153}$. Evaluation of baroreflex function by spectral methods is based on the concept that each spontaneous oscillation in blood pressure elicits an oscillation at the same frequency in R-R interval by the effect of arterial baroreflex activity ${ }^{138}$. As found in other fetal sheep experiments, we observed an oscillation frequency associated with fetal baroreflex activity centered around 0.1 $\mathrm{Hz}^{154}$. According to the transfer function method, baroreflex sensitivity was calculated as the value of the transfer function modulus and phase (i.e. gain and delay) between SBP and R-R interval in the LF frequency, considering a coherence $\geq 0.5^{149}$. The transfer function methodology has been used in several studies of human preterm infants ${ }^{142,150}$. As the values of SBP and R-R intervals obtained from the fetal sheep are comparable with values found in preterm infants, the same spectral analysis methodology was followed. Transfer function analysis in this study showed relatively high coherence values in the LF band, suggesting reliable estimates of transfer gain and delay.

Long-term effects of hypoxic-ischemia During longitudinal monitoring of the UCO group, the LF transfer gain gradually decreased over time, leading to a significantly lower LF transfer gain post-UCO compared to the control group. Consistent with this finding is the time-domain observation of a decreased SD-RR, while SD-SBP remains unchanged. In contrast, the LF delay between blood pressure variations and subsequent heart rate variations increased compared to the control group. The gradual decrease of baroreflex sensitivity and increase in delay between variations in heart rate and systolic blood pressure suggest that hypoxic-ischemia results in a deregulation of the baroreceptor reflex arc. A decreased LF transfer gain and increased delay may result from changes in the dynamics of the sympathetic and parasympathetic pathways and/or from changes in the balance between these pathways ${ }^{137,145,147,153}$. As the parasympathetic effect on heart rate changes is fast compared to sympathetic effect, increased delay may indicate a greater relative contribution of sympathetic regulation. This is supported by the finding that normalized LF power (LF/total power) increased to nearly $100 \%$ following UCO, while remained stable in controls (approximately $75 \%$ ).

The afferent signals from the baroreceptor output are projected on the nucleus of the solitary tract, which is located in the medulla region, to be processed and transferred to the blood pressure regulation effectors ${ }^{155}$. Previous findings in 0.6 gestation fetal sheep showed that suppressed fetal heart rate variability following 30 minute UCO was associated with severe neuronal loss in the brain stem 3 days after the insult ${ }^{87}$. This neuronal loss may affect the processing of afferent nerve signals and therefore the baroreflex function, thereby decreasing regulation efficacy. Although we did not study brain stem histology, our findings showed that deregulation of baroreflex 
function gradually increases over the course of seven days suggesting evolving neuronal loss in the brain stem. This finding also highlights the importance of study length. At 3 days after UCO, the end-point for most other longitudinal studies ${ }^{87,148}$, baroreflex transfer function parameters of our UCO group were not significantly different from the control group, potentially obscuring long-term hypoxic-ischemia-induced functional loss. We speculate that gradual functional loss may be related to the evolvement of hypoxic-ischemic associated cytotoxic edema during the first week as seen by MRI studies in human infants ${ }^{156}$. Fetal heart rate variability (SD-RR) was already significantly decreased at 3 days post-UCO, and gradually decreased further during the remainder of the study. This observation is in agreement with Ikeda et al., who showed that decreased long-term fetal heart rate variability at 24-72 $\mathrm{h}$ of recovery after UCO showed to be an indicator of the severity of histological damage in the term fetal brain ${ }^{157}$. To our knowledge, our study is the first to describe this change in fetal heart rate variability in preterm asphyxiated fetal lambs.

Maturational effects of baroreflex activity Results of our study indicate that the baroreflex function matures during fetal life, as shown by the increase in LF transfer gain for the control group between 0.75 and $0.80 \mathrm{GA}$. These results confirm findings by other experiments, which noted that the baroreflex function was active from approximately 85 days of gestation $(0.6 \mathrm{GA})$, and the magnitude of fetal heart rate response to blood pressure changes increased towards term ${ }^{144}$. Other studies found a decrease in baroreflex sensitivity during development ${ }^{158-160}$. Several studies showed no significant maturation of the baroreflex sensitivity ${ }^{145,146}$. There may be several explanations for these conflicting findings. First, it is known that the different pathways of the baroreflex feedback system mature at different rates. In preterm sheep of 0.7 GA there is probably no significant control by the renal sympathetic nerve, which regulates vascular resistance ${ }^{147,161}$, whereas in the term sheep vascular resistance control is present ${ }^{159}$. In our study, fetal sheep were instrumented at $0.7 \mathrm{GA}$, and maturational changes were studied between 0.75 and $0.80 \mathrm{GA}$. Other studies have little to no variation in GA, or their results lack the temporal resolution required to compare results to our findings directly $145,146,158,159$. Second, the discrepancies may be due to methodological differences. Quantification of spontaneous baroreflex sensitivity by spectral methods and quantification by injection of drugs or aortic compression do not necessarily produce comparable results ${ }^{162}$. Spontaneous variations in blood pressure are normally in the order of several $\mathrm{mmHg}$, whereas experiments using invasive quantification methods generally study heart rate responses to larger blood pressure changes. Third, heart rate response to blood pressure changes is non-linear, and can be characterized by a sigmoidal input-output relation with maximum responses around the (normal) operating point and decreased regulation capacity at the extremes ${ }^{139}$.

Pathophysiological implications Reduction in fetal heart rate variability is associated with fetal hypoxia and acidemia in human preterm ${ }^{163}$ and term fetus ${ }^{164}$. However, despite the highly adverse outcomes of moderate to severe encephalopathy around birth the predictive value of abnormal fetal heart rate patterns for cerebral palsy is consistently weak ${ }^{165}$. This study demonstrates an association between fetal hypoxicischemia and reduced baroreflex sensitivity. For obvious reasons, direct measurement of fetal blood pressure in humans is not possible. Future studies may explore the use of non-invasive (Doppler) estimation of fetal blood pressure and spectral-derived 
variability in assessing hypoxemia-induced changes in brain stem mechanisms for cardiovascular control $^{166}$.

\section{Conclusion}

In conclusion, this is the first study to quantify baroreflex mediated heart rate response in a preterm fetal sheep model by transfer function analysis of R-R interval and SBP fluctuations, and to show long-term effects on baroreflex function after intrauterine umbilical cord occlusion induced hypoxic-ischemia. Our results show that intrauterine hypoxic-ischemia limits baroreflex efficacy to buffer changes in SBP by adapting fetal R-R interval following umbilical cord occlusion. As a consequence, the preterm brain may be prone to blood pressure fluctuations, possibly leading to disruption of the immature vascular architecture and augmenting already present cerebral damage after systemic hypoxic-ischemia in the reperfusion period. 



\section{Baroreceptor reflex function in HIE with stem cell treatment}

Submitted:

Jellema, R. K., Ophelders, D. R. M. G., Zwanenburg, A., Nikiforou, M., Delhaas, T., Andriessen, P., Mays, R. W., Deans, R. W., Germeraad, W. T. V., Wolfs, T. G. A. M. \& Kramer, B. W. Clinical-grade Multipotent Adult Progenitor Cells for Hypoxic-Ischemic Injury in the Preterm Brain. J Neuroinflammation (2015)

Note: A. Zwanenburg made major contributions to acquisition, analysis and interpretation of physiological signals 



\begin{abstract}
Background Preterm infants are at risk for hypoxic-ischemic encephalopathy (HIE). No therapy exists to treat this brain injury and subsequent long-term sequelae. We have previously shown in a wellestablished preclinical model of global hypoxia-ischemia (HI) that mesenchymal stem cells (MSCs) are a promising candidate for the treatment of hypoxic-ischemic brain injury. In the current study we investigated the neuroprotective capacity of multipotent adult progenitor cells (MAPCs), which are a subset of bone marrow-derived MSCs with more potent anti-inflammatory and regenerative properties.
\end{abstract}

Methods Instrumented preterm sheep fetuses were subjected to global hypoxiaischemia by 25 minutes of umbilical cord occlusion at a gestational age of 106 (term 146) days. During a 7-day reperfusion period, vital parameters (e.g. blood pressure and heart rate; baroreceptor reflex) and (amplitude-integrated) electroencephalogram were recorded. At the end of the experiment, the preterm brain was studied by histology.

Results Systemic administration of MAPCs reduced the number and duration of seizures and prevented decrease in baroreflex sensitivity after global HI. In addition, MAPCs prevented $\mathrm{HI}$-induced microglial proliferation in the preterm brain. These antiinflammatory effects were associated with MAPCinduced prevention of hypomyelination after global HI. Besides attenuation of the cerebral inflammatory response, our findings showed that MAPCs modulated the peripheral splenic inflammatory response, which has been implicated in the etiology of hypoxic-ischemic injury in the preterm brain.

Conclusion In a preclinical animal model MAPCs improved the functional and structural outcome of the preterm brain after global HI. Future studies should establish the mechanism and long-term therapeutic effects of neuroprotection established by MAPCs in the developing preterm brain exposed to HI. Our study may form the basis for future clinical trials, which will evaluate whether MAPC therapy is capable of reducing neurological sequelae in preterm infants with HIE. 


\section{Introduction}

Hypoxia-ischemia ( $\mathrm{HI})$ in the developing brain is strongly correlated with mortality and neurological morbidity in preterm and full-term infants, resulting in an enormous physical, psychological and economic burden. Unfortunately, no therapeutic cure is available for hypoxic-ischemic brain injury in preterm infants.

In a translational animal model of global $\mathrm{HI}$ in the preterm ovine fetus, our group has demonstrated that cell-based therapy may be a promising neuroprotective strategy for preterm neonates suffering from $\mathrm{HI}$-induced brain injury ${ }^{70}$. We showed that intravenous administration of exogenous mesenchymal stem cells (MSCs) protected against functional loss and structural injury of the preterm brain ${ }^{70}$. These neuroprotective effects were largely attributable to attenuation of (neuro) inflammatory processes, as indicated by decreased microglial activation and proliferation in the preterm brain and induction of peripheral T-cell tolerance, which was associated with reduced cerebral infiltration of these immune effector cells ${ }^{70}$.

Multipotent adult progenitor cells (MAPCs), a subpopulation of bone marrowderived progenitor cells have high expansion potential and their immunological properties make it possible to use them as a universal allogeneic donor ${ }^{167-169}$. Pre-clinical animal studies have demonstrated that in comparison to MSCs, MAPCs have stronger anti-inflammatory effects and are more potent in promoting endogenous tissue regeneration after ischemic and traumatic brain injury ${ }^{64-66,170,171}$. In addition, the smaller size of MAPCs compared to MSCs facilitates passage through the pulmonary capillary bed, which may increase availability of MAPCs in the systemic and cerebral vasculature and thus enhancing their neuroprotective effect ${ }^{172}$.

Based on these superior qualities of MAPCs, we aimed to assess the antiinflammatory and neuroprotective potential of MAPCs in the preterm brain exposed to global HI. We hypothesized that systemic administration of a clinical-grade MAPCs product, would attenuate cerebral and peripheral inflammation and prevent structural and functional brain injury after global $\mathrm{HI}$ in the preterm ovine fetus. We tested our hypothesis in a well-established pre-clinical animal model of preterm global HI. In this model, preterm ovine fetuses were exposed to global $\mathrm{HI}$ by transient umbilical cord occlusion (UCO) at 0.7 gestation which is equivalent to 30-32 weeks human gestation ${ }^{173}$, followed by systemic administration of MAPCs during a 7 day reperfusion period.

The anti-inflammatory effects of MAPCs were assessed in the preterm brain by analysis of microglial responses in the hippocampus and subcortical white matter. The spleen was assessed, since previous reports suggest that MAPC-induced alterations in the splenic inflammatory response may be responsible for their neuroprotective effect after brain injury ${ }^{64}$. Structural effects of systemic MAPC administration were assessed by histological white matter injury examination. Brain function was studied by analysis of cortical function by means of electrographic seizure activity and central (brain stem) function by means of baroreflex sensitivity. 


\section{Methods}

\section{Animal experiment}

The experimental protocol and study design were in line with the institutional guides for animal experiments and were approved by the institutional Animal Ethics Research committee of Maastricht University, the Netherlands.

Randomization and blinding Thirty-two singleton fetuses of time-mated Texel ewes were randomized by an independent researcher who was not involved in the animal experiments. Randomization resulted in four experimental groups: (1) sham umbilical cord occlusion, saline treatment (sham-SAL $n=8$ ), (2) sham umbilical cord occlusion, MAPC treatment (sham-MAPC; $n=8)$, (3) umbilical cord occlusion, saline treatment (HISAL; $n=8$ ) and (4) umbilical cord occlusion, MAPC treatment (HI-MAPC; $n=8$ ) (Figure 7.1). The investigator performing the (sham) umbilical cord occlusions was blinded to treatment allocation. Tissue sampling and the analyses of brain tissue and electrophysiological data were conducted in a blinded fashion.

Animals and surgery Singleton fetuses were surgically instrumented at 102 days gestational age (term 147 days) as described previously ${ }^{29}$. Briefly, fetuses were partially exposed through a midline incision. A polyurethane umbilical vessel catheter $(1.2 \mathrm{~mm}$, Covidien, Mansfield, Massachusetts, USA) was placed in the left femoral artery and brachial vein for blood pressure recordings, blood sampling, and administration of MAPC. An additional catheter was placed into the amniotic sac for measurement of amniotic fluid pressure. Three electrocardiogram (ECG) electrodes were placed on the fetal thorax for cardiac monitoring. Two pairs of custom-made shielded silver-tipped electroencephalogram (EEG) electrodes (Cooner wire Co., Chatsworth, CA, USA) were placed bilaterally on the dura over the parasagittal parietal cortex with a subcutaneous silver reference electrode placed in the neck. An inflatable vascular occluder (OC16HD,

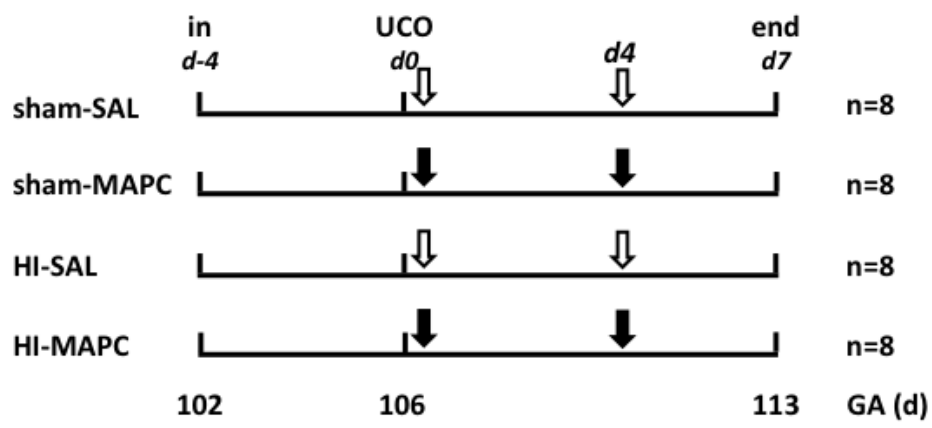

Figure 7.1: Study design. Fetuses were instrumented at a gestational age (GA) of $102 \mathrm{~d}$. After a recovery period of $4 \mathrm{~d}$ fetuses were subjected to 25 min of umbilical cord occlusion (UCO) or sham. One hour and 4 days after UCO or sham, fetuses received either intravenous MAPCs (10 million cells, closed arrow) or saline $0.9 \%$ (open arrow). After a $7 d$ reperfusion period brain tissue was collected. Abbreviations: in = instrumentation, $\mathrm{HI}=$ hypoxiaischemia, $S A L=$ saline, $M A P C S=$ multipotent adult progenitor cells. 


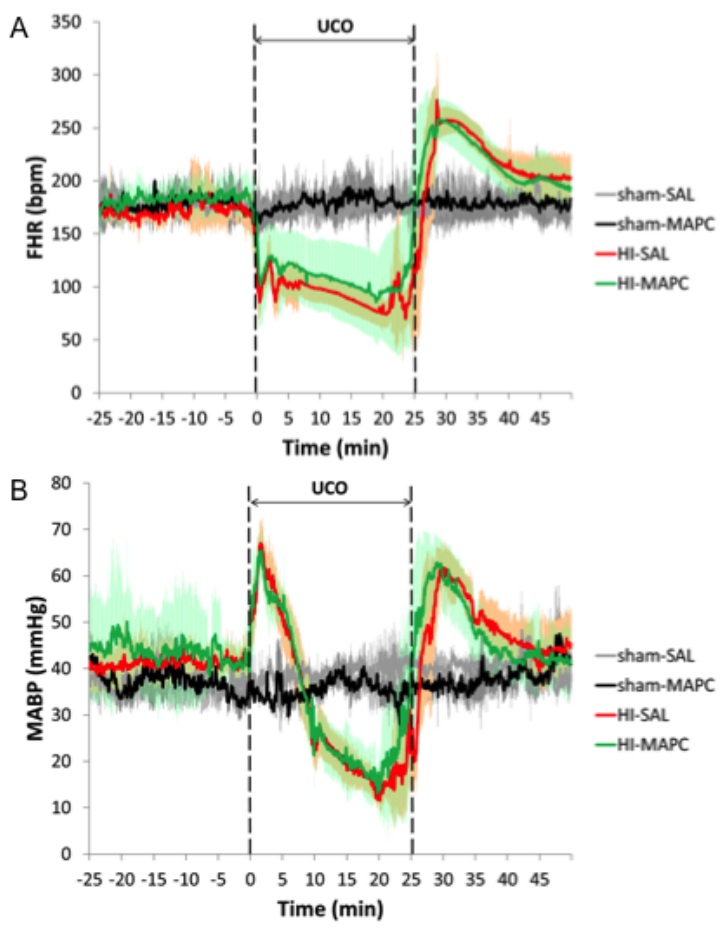

Figure 7.2: Reproducibility of 25 min umbilical cord occlusion (UCO) as evidenced by comparable vital parameters and blood gases in animals exposed to global HI. (A) Fetal heart rate (FHR) and $(B)$ fetal mean arterial blood pressure (MABP) measurements indicated that all animals exposed to global $\mathrm{HI}$ experienced the same degree of bradycardia and hypotension, respectively; means (thick line) $\pm S D$ (shaded areas) of $n=8$ animals per experimental group are shown. $H I=$ hypoxia-ischemia, $S A L=$ saline, $M A P C S=$ multipotent adult progenitor cells, $\min =$ minutes .

$16 \mathrm{~mm}$, In Vivo Metric, Healdsburg, CA, USA) was placed around the umbilical cord for induction of transient global HI. All fetal leads were exteriorised through the maternal flank. Post-operatively sheep were housed individually with access to water and food ad libitum. A period of 4 days post-operative recovery was incorporated before onset of the experiments.

Experimental design At 106 days gestational age (experimental day 0) fetuses were subjected to 25 minutes of (sham) umbilical cord occlusion by rapidly inflating the occluder with sterile saline of a defined volume known to completely inflate the occluder. An acute drop in heart rate and a gradual decline in blood pressure conformed complete occlusion of the umbilical cord (Figure 7.2). Global hypoxia-ischemia was further monitored with subsequent arterial blood gas analysis indicating acidemia, hypoxemia and hypercapnia (data not shown). One hour after umbilical cord occlusion and on experimental day 4 fetuses received either an intravenous bolus of MAPCs or an equal volume of vehicle. At the end of the experiment (experimental day 7), both ewe and fetus were euthanised by administration of pentobarbital $(200 \mathrm{mg} / \mathrm{kg})$. 


\section{Data acquisition and analysis}

Physiological data Physiological data was sampled as described previously on a custom-made MPAQ unit (Maastricht-Programmable AcQuisition system, Maastricht Instruments BV, Maastricht, the Netherlands) with IDEEQ software (Maastricht Instruments BV ${ }^{29}$. In short, EEG data were sampled at $1000 \mathrm{~Hz}$ and stored on hard-disk for off-line analysis. EEG data were filtered using a $0.5-30 \mathrm{~Hz} 4^{\text {th }}$ order Butterworth band-pass filter. EEG signal with an amplitude $>1000 \mu \mathrm{V}$ was considered an artifact and removed from analysis ( $<1 \%$ of data). After filtering, the raw EEG signals of the central and posterior channels were converted into amplitude-integrated EEG (aEEG) traces, using a MATLAB-based algorithm similar to the clinical EEG NicoletOneTM device (Viasys Healthcare, Conshohocken, PA, USA), as previously described ${ }^{69,70,127,129,174}$. EEG seizure activity was annotated manually using aEEG/EEG traces and the total number and length of seizures was subsequently calculated. A neonatologist experienced in aEEG interpretation and blinded to treatment allocation, performed annotation.

ECG and blood pressure data were processed off-line using MATLAB R2013a (The MathWorks, Inc., Natick, MA). The ECG recordings were used to determine R-R intervals. Blood pressure measurements were corrected for amniotic pressure and used to determine the mean arterial blood pressure in each pressure wave. As previously reported, heart rate and heart rate variability were assessed in $192 \mathrm{~s}$ segment by calculating the mean R-R interval and standard deviation (SD-RR) respectively ${ }^{142,150}$. Likewise, mean systolic blood pressure and blood pressure variability was assessed by calculating the mean SBP and standard deviation (SD-SBP) ${ }^{150}$. As a time series estimate of baroreflex sensitivity, we calculated the ratio between SD-RR and SD-SBP ${ }^{150,151,175}$.

MAPCs Multipotent Adult Progenitor Cells were provided by Athersys, Inc. (Cleveland $\mathrm{OH}, \mathrm{USA}$ ) and stored in liquid nitrogen. Cells were prepared under good manufacturing practice (GMP) and genomic and epigenetic stability of the cells were confirmed as previously described ${ }^{167,176}$. Flow cytometric analysis of cell-surface proteins demonstrated that the cells were CD29, CD90, CD44 positive, and MHC class I low, and were negative for MHC class II, CD45, CD106, and the costimulatory molecules CD80 and CD86. Cells were able to differentiate into mesenchymal cell types (adipocytes, osteoblasts, chondrocytes, and smooth muscle cells), but also towards endothelium ${ }^{169}$. Prior to injection, MAPCs were thawed and washed twice and suspended in phosphate buffered saline (PBS) at a concentration of $10 \cdot 10^{6}$ cells $/ \mathrm{mL}$. MAPCs were administered intravenously 1 hour and 4 days after (sham) UCO. Fetuses received two doses of 10 . $10^{6}$ cells in $1 \mathrm{~mL}$ PBS.

Immunohistochemistry brain After 3 months of submersion fixation in $4 \%$ paraformaldehyde, the right cerebral hemisphere was embedded in gelatin and serial coronal sections $(50 \mu \mathrm{m})$ were cut on a Leica VT 1200 S vibrating microtome (Leica Biosystems, Nussloch, Germany). Free floating sections at the level of mid-thalamus and posterior hippocampus were stained with a rabbit anti-ionized calcium binding adaptor molecule 1 (IBA-1) antibody (Wako Pure Chemical Industries, Osaka, Japan) for microglia or a rat anti-myelin basic protein (MBP) antibody (Merck Millipore, Billerica, MA, USA) for myelin sheaths and myelin producing (mature) oligodendrocytes, as previously described $^{69}$. 
Briefly, endogenous peroxidase-activity was blocked by incubation with $0.3 \% \mathrm{H}_{2} \mathrm{O}_{2}$ in Tris Buffered Saline (TBS, pH 7.6). Free floating sections were incubated overnight at $4{ }^{\circ} \mathrm{C}$ with the diluted primary antibody (1:1000 anti-IBA-1, 1:2000 MBP) followed by incubation with the appropriate secondary antibodies. The immunostaining was enhanced with Vectastain ABC peroxidase Elite kit (PK-6200, Vector Laboratories, Burlingame, CA, USA) followed by a nickel sulfate-diaminobenzidine (NiDAB) staining. Sections were mounted on gelatin-coated glass slides, air-dried, dehydrated in ascending ethanol concentrations and coverslipped with PerTex.

Analysis of immunohistochemistry Immunohistochemical stainings were analyzed as previously described $^{69}$. For the analysis of IBA-1 and MBP immunoreactivity, digital images of the subcortical white matter (SCWM) (100x magnification) and hippocampus (20x magnification; only IBA-1) were acquired using an Olympus AX-70 microscope (Olympus, Tokyo, Japan) equipped with a black and white digital camera. In the hippocampus, area fraction of IBA-1 immunoreactivity was assessed in one digital image per section by delineating the hippocampus and determining the areal fraction of IBA-1 immunoreactivity expressed as a percentage of total hippocampal area with a standard threshold using Leica Qwin Pro V 3.5.1 software (Leica, Rijswijk, the Netherlands). In the SCWM, the area fractions of IBA-1 and MBP immunoreactivity were determined in five adjacent 100x digital images obtained in standardized locations within the SCWM of each section. The results of five images per section were averaged to obtain the areal fractions of IBA-1 and MBP immunoreactivity within the SCWM for each section.

RNA extraction and quantitative real-time PCR For real-time (RT) quantitative polymerase chain reactions ( $q P C R$ ), total RNA was extracted from spleen using the SV Total RNA isolation system (Z3100; Promega, Madison, WI) according to the manufacturer's recommendations. RT qPCR reactions were performed in duplicate with the SensiMix SYBR No-ROX kit (QT650-02; Bioline Reagents Ltd) in a LightCycler 480 Instrument with ovine specific primers for interleukin IL-10 (5'-

CATGGGCCTGACATCAAGGA-3' (sense), 5'-CGGAGGGTCTTCAGCTTCTC-3' (antisense). RT qPCR results were normalized to the housekeeping gene ovine $40 \mathrm{~S}$ ribosomal protein S15 (ovRPS15) (5'-CGAGATGGTGGGCAGCAT-3' (sense), 5'-GCTTGATTTCCACCTGGTTGA-3' (antisense)).

\section{Statistics}

Summary statistics of animal characteristics and all outcome variables are shown as means with 95\% Confidence Interval (CI). Groups' comparisons of all outcome parameters were drawn with analysis of variance (ANOVA) or with random intercept models in case of repeated measurements per animal (e.g. different sections per brain). $\mathrm{HI}$ (sham vs. $\mathrm{HI}$ ) and treatment (saline vs. MAPC) were the fixed effects. For random intercept models, animals constituted additionally the random effect. Variables, whose distributions were positively skewed, were log-transformed previous to statistical testing. To facilitate interpretation, averages on the log scale were back transformed to the original scale (antilog) and were presented as geometric means and corresponding $95 \%$ Cls.

Seizure data, measured over time, showed pronounced right-skewness, caused by the absence of seizures in non-hypoxic conditions, that could not be remedied by log 
transformation. Hence, for these variables, pair-wise groups' comparisons were performed with non-parametric Mann-Whitney $U$ tests, per individual time-point. They are presented as medians and corresponding interquartile range (IQR). A False Discovery Rate (FDR) of $5 \%$ was used for multiple testing correction. Groups' differences with FDR corrected $p<0.05$ were considered statistically significant. Statistical analysis was performed with IBM SPSS Statistics Version 20.0 (IBM Corp., Armonk, NY, USA).

Statistical analysis of baroreflex data was performed using a Bayesian multilevel mode $\mathrm{l}^{177}$ to estimate the daily effects of global $\mathrm{HI}$ and MAPC treatment under hypoxic and sham conditions on the baroreflex sensitivity (Appendix A). This model corrects for repeated measurements and takes into account variance between and within subjects $^{177}$. Statistical time series analysis was conducted using the Stan for R package (version 2.6.0) in $\mathrm{R}$ (version 3.1.1).

\section{Results}

\section{Animal characteristics}

To test the neuroprotective potential of clinical-grade MAPCs, we randomized 32 preterm sheep fetuses in four different experimental groups (Figure 7.1). After instrumentation and a recovery period of four days, animals were subjected to $25 \mathrm{~min}$ of (sham) umbilical cord occlusion (UCO) to induce global hypoxia-ischemia (HI). MAPCS were administered 1 hour and 4 days after UCO (Figure 7.1). Fetal body weight and gestational age at did not differ significantly between the four experimental groups (Table 7.1). In line with previous reports ${ }^{29,69,70}$, the fetal response to hypoxia-ischemia was comparable in all animals exposed to UCO (Figure 7.2), indicating that the injury group (HI-SAL) and intervention group (HI-MAPC) were exposed to a similar degree of global hypoxia-ischemia.

\section{MAPCs reduced the cerebral inflammatory response after global HI}

The cerebral inflammatory response was studied by assessing microglial proliferation in the subcortical white matter and hippocampus using ionized calcium binding adaptor molecule 1 (IBA-1), which is a highly specific marker for resting and activated microglia in sheep ${ }^{29}$. Global $\mathrm{HI}$ resulted in a significant (sham-SAL vs. HI-SAL; $p$ $<0.001$ ) increase of IBA-1 immunoreactivity in the SCWM and hippocampus, indicating profound microglial proliferation in these regions (Figure 7.3). MAPCs significantly (HISAL vs. HI-MAPC; $p=0.041$ ) reduced IBA-1 immunoreactivity in the SCWM (Figure 7.3A and $C$ ). MAPCs did not reduce IBA-1 immunoreactivity in the hippocampus (HI-SAL vs. HI-MAPC; $p=0.883$ ) (Figure 7.3B and D). No differences in IBA-1 immunoreactivity were observed between saline or MAPC treated sham operated animals in the SCWM and hippocampus. 


\begin{tabular}{lrrrr} 
& Sham-SAL & Sham-MAPC & HI-SAL & HI-MAPC \\
\hline GA at UCO (d) & 105.2 & 106.0 & 105.5 & 105.2 \\
BW (g) & $(105.0-105.5)$ & $(105.3-106.7)$ & $(105.1-106.0)$ & $(104.9-105.8)$ \\
& 1690 & 1532 & 1542 & 1559 \\
& $(1516-1865)$ & $(1245-1820)$ & $(1362-1721)$ & $(1340-1779)$
\end{tabular}

Table 7.1: Animal characteristics. Fetuses were subjected to umbilical cord occlusion (UCO) at a comparable gestational age (GA). Fetal body weight (BW) did not differ between experimental groups. Means $\pm 95 \% \mathrm{Cl}$ are shown. Groups' comparisons were drawn with ANOVA (sham-SAL $n=8$, sham-MAPC $n=8, H I-S A L n=8, H I-M A P C n=8$ ). $H I=$ hypoxia-ischemia, $S A L=$ saline,$M A P C S=$ multipotent adult progenitor cells.

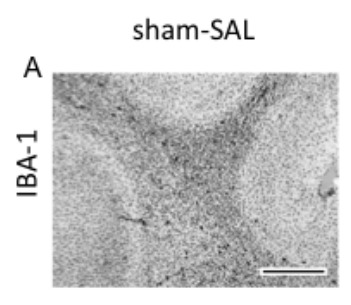

sham-MAPC
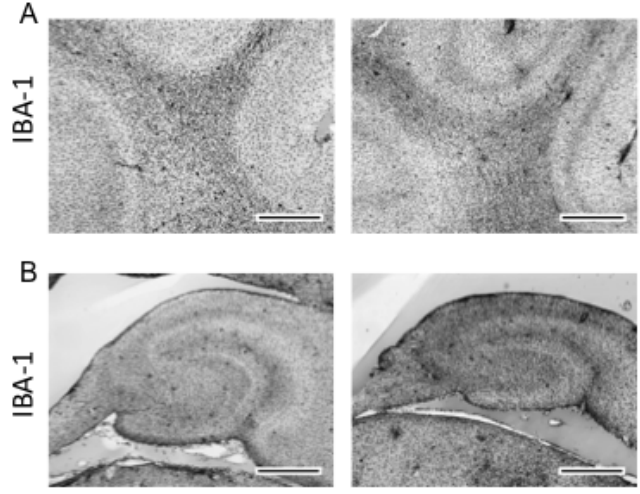

subcortical white matter

C

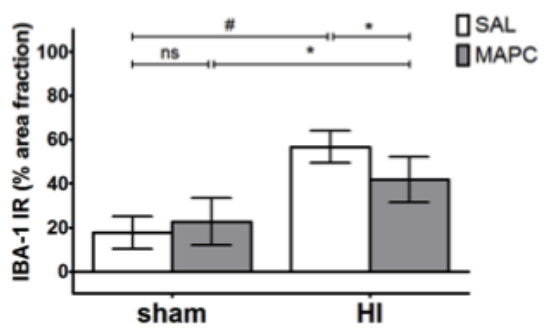

HI-SAL
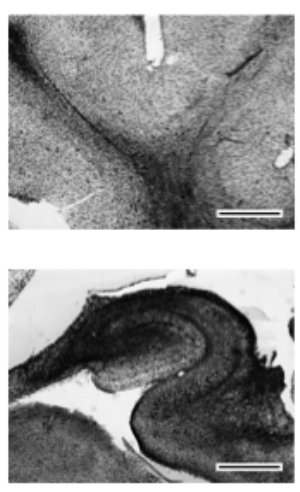

hippocampus

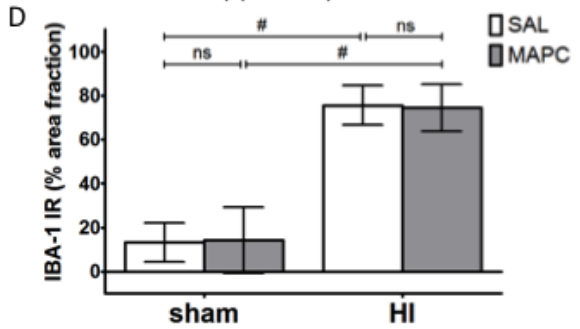

HI-MAPC
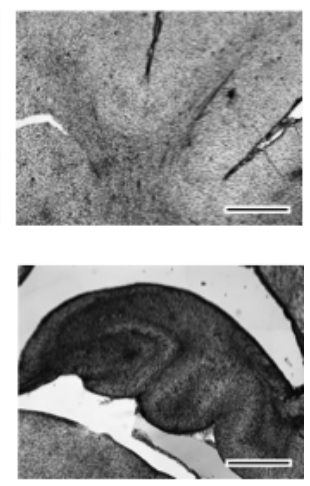

Figure 7.3: MAPCs reduced cerebral inflammation in the subcortical white matter (SCWM), but not in the hippocampus. (A-B) Immunohistochemical IBA-1 staining in the SCWM and hippocampus of the four experimental groups. Global HI induced a profound increase of IBA-1 immunoreactivity in both regions, which was significantly reduced by MAPCs in the SCWM, but not in the hippocampus. (C-D) Graphical presentation of area fraction of IBA-1 immunoreactivity in SCWM and hippocampus. Means $\pm 95 \% \mathrm{Cl}$ and levels of significance are depicted, which were calculated by the random intercept model with all repeated measures (i.e. brain sections) per animal (sham-SAL $n=8$, sham-

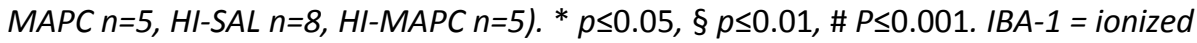
calcium binding adaptor molecule $1, H I=$ hypoxia-ischemia, $S A L=$ saline, $M A P C=$ multipotent adult progenitor cells, $I R=$ immunoreactivity. $(A-B)$ Scale bars represent 1 $\mathrm{mm}$. 


\section{MAPCS prevented HI-induced white matter injury}

White matter injury was studied by assessing myelin basic protein (MBP) immunoreactivity in the SCWM. Global HI significantly (sham-SAL vs. HI-SAL; $p<0.001$ ) reduced $\mathrm{MBP}$ immunoreactivity in the SCWM, indicating $\mathrm{HI}$-induced hypomyelination in this region (Figure 7.4A and $B$ ). MAPCs significantly increased MBP immunoreactivity (HI-SAL vs. HI-MAPC; $p=0.015$ ) No differences in MBP immunoreactivity in the SCWM were observed between sham animals treated with saline or MAPCs. We determined brain weight as a surrogate for neuronal injury (Figure 7.4C). Brain weight was significantly reduced after global HI (sham-SAL vs. HI-SAL; $p=0.042$ ). MAPCs partially prevented loss of brain weight, but the effect did not reach significance (HI-SAL vs. HIMAPC; $p=0.423$ ).

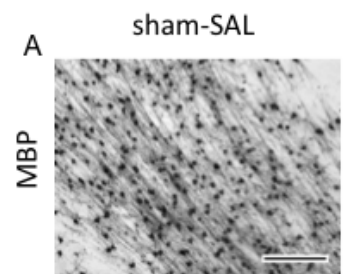

sham-MAPC

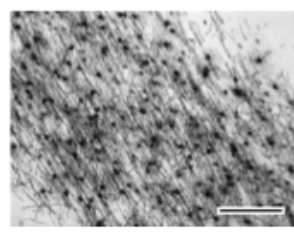

B

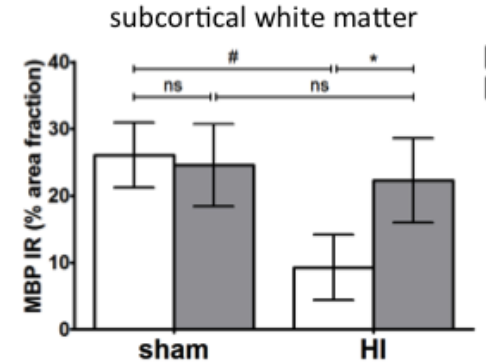

DSAL IMAPC
HI-SAL

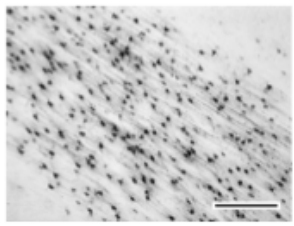

HI-MAPC

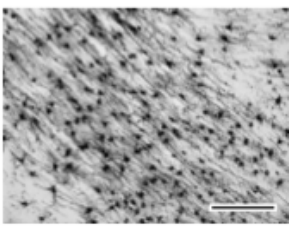

brain weight

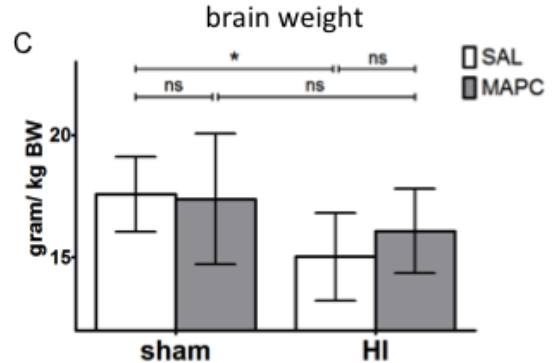

Figure 7.4: MAPCs reduced white matter injury after global HI. (A) Immunohistochemical MBP staining in the SCWM of the four experimental groups. Global HI induced marked hypomyelination in the SCWM. MAPCs significantly prevented the decrease in $M B P$ reactivity after global $H I$. The area fraction of $M B P$ was similar in sham conditions. (B) MBP immunoreactivity in SCWM; means $\pm 95 \% \mathrm{Cl}$ and levels of significance are depicted, which were calculated by the random intercept model with all repeated measures (i.e. brain sections) per animal (sham-SAL $n=8$, sham-MAPC $n=5, H$ $S A L n=8$, HI-MAPC $n=5$ ). (C) Brain weight corrected for body weight (BW) in the four experimental groups; means $\pm 95 \% \mathrm{Cl}$ and levels of significance are depicted, which were calculated with ANOVA (sham-SAL $n=8$, sham-MAPC $n=8, H I-S A L n=8, H I-M A P C$ $n=8) .{ }^{*} p \leq 0.05, \S p \leq 0.01, \# p \leq 0.001 . M B P=$ myelin basic protein $1, H I=$ hypoxiaischemia, $S A L=$ saline, $M A P C S=$ multipotent adult progenitor cells, $I R=$ immunoreactivity. (A-B) Scale bars: $200 \mu \mathrm{m}$. 

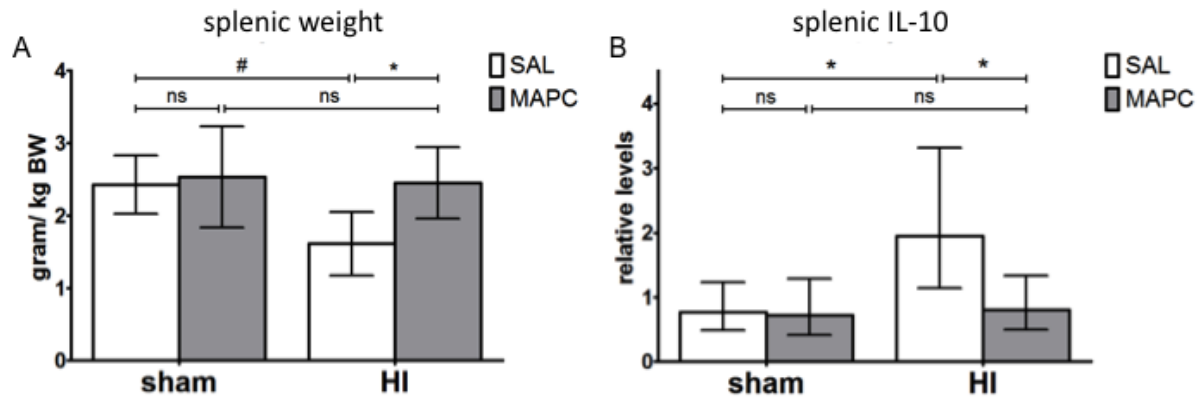

Figure 7.5: MAPCs prevented splenic involution and modulated the peripheral inflammatory response. (A) Splenic weight corrected for body weight (BW). Global HI induced significant splenic involution, which was significantly prevented by MAPCs; means $\pm 95 \% \mathrm{Cl}$ and levels of significance are depicted, which were calculated with ANOVA (sham-SAL n=8, sham-MAPC $n=8, H I-S A L n=8, H I-M A P C n=8$ ). (B) IL-10 mRNA levels were significantly increased in the spleen 7 days after global HI. MAPCS significantly attenuated the HI-induced IL-10 response in the spleen; geometric means \pm 95\% $\mathrm{Cl}$ and levels of significance are depicted, which were calculated with ANOVA (sham-SAL $n=8$, sham-MAPC $n=6$, HI-SAL $n=8$, HI-MAPC $n=8$ ). * $p \leq 0.05, \S p \leq 0.01$, \# $p \leq 0.001$. $H I=$ hypoxia-ischemia,$S A L=$ saline, $M A P C=$ multipotent adult progenitor cells.

\section{MAPCs prevented splenic involution and modulated the peripheral inflammatory response}

Splenic weight was assessed, since activation of the splenic inflammatory response was previously shown to be characterized by mobilization of effector immune cells affecting volume and weight of the spleen ${ }^{29}$. Global $\mathrm{HI}$ induced splenic involution in saline treated animals (sham-SAL vs. HI-SAL, $p=0.021$ ) (Figure 7.5). MAPCs significantly prevented splenic involution after global HI (HI-SAL vs. HI-MAPC, $p=0.030$ ). Splenic IL10 mRNA levels were determined since previous findings indicated that MAPCs established neuroprotection by modulating the splenic inflammatory response in an IL10 mediated manner. Splenic IL-10 mRNA levels were significantly increased after global $\mathrm{HI}$ (sham-SAL vs. HI-SAL, $p=0.029$ ). MAPCs significantly reduced IL-10 mRNA levels in the spleen after global HI (HI-SAL vs. HI-MAPC, $p=0.039$ ).

\section{MAPCs reduced seizure burden after global HI}

Electrographic seizure activity was assessed to determine the effect of MAPCs on cortical function after global $\mathrm{HI}$. Global $\mathrm{HI}$ induced a significant increase in total number and duration of seizures after global HI (sham-SAL vs. HI-SAL, significant in all time points) (Figure 6). Seizure burden peaked within the first 48 hours following global HI with a second small rise in activity around experimental day 4 and 5 (Figure 7.6). MAPCs reduced seizure burden throughout the complete reperfusion period (HI-SAL vs. HIMAPC, significant from experimental day 2 onwards). Cumulative number of seizures in the complete reperfusion study was significantly increased after global HI (sham-SAL vs. HI-SAL, $p=0.015$ ) and significantly reduced by MAPCs (HI-SAL vs. HI-MAPC, $p=0.021$ ). Similarly, cumulative duration of seizures was significantly increased after global $\mathrm{HI}$ 
(sham-SAL v. HI-SAL, $p=0.014$ ) and significantly reduced by MAPCs (HI-SAL vs. HIMAPC, $p=0.041$ ). No electrographic seizures activity was detected under sham conditions.

\section{MAPCs prevented loss of baroreflex sensitivity after global $\mathrm{HI}$}

Baroreceptor reflex sensitivity was analyzed to study the effect of MAPC treatment on deeper brain functions situated in the brain stem (Figure 7.7). Global HI increasingly compromised baroreceptor reflex sensitivity over time (sham-SAL vs. HI-SAL, significant for all time points with the exception of experimental day 1). MAPCs significantly prevented the loss of baroreflex sensitivity (HI-SAL vs. HI-MAPC, significant on all time points).
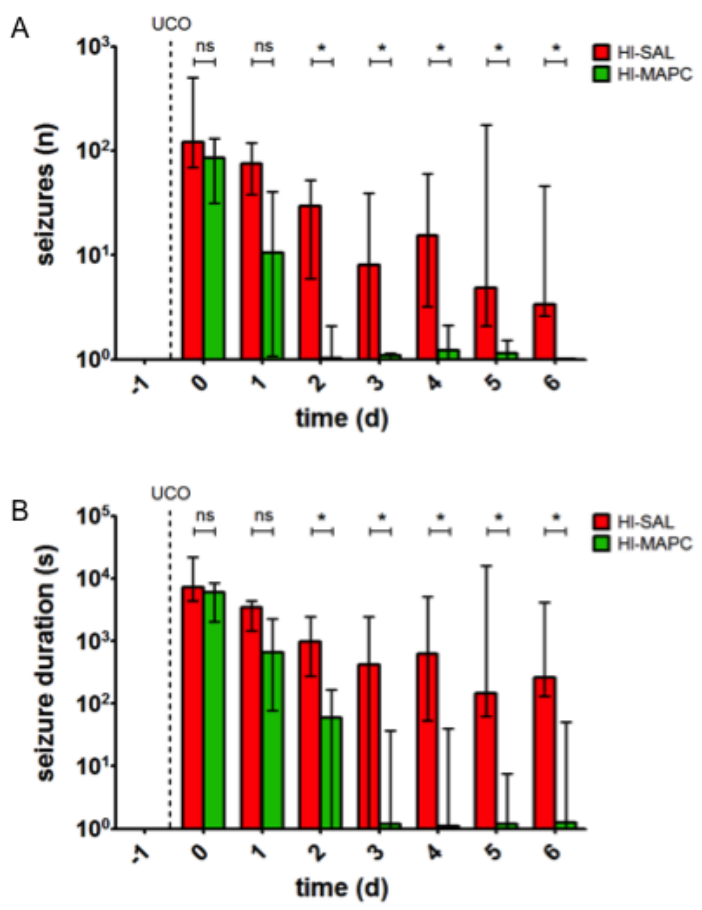

Figure 7.6: MAPCs induced functional neuroprotection after global HI. Global $\mathrm{HI}$ caused a significant seizure burden indicated by an increased (A) total number and (B) duration of seizures compared to controls. Administration of MAPCs significantly reduced electrographic seizure number and duration. Medians \pm interquartile ranges (IQR) and levels of significance of the treatment effect (HI-SAL vs. HI-MAPC) are depicted, which were calculated by Mann-Whitney test (HI-SAL $n=8, H I-M A P C n=8)$. No electrographic seizure activity was detected under sham conditions. For readability purposes these sham groups (sham-SAL $n=8$ and sham-MAPC $n=6$ ) are not shown. ${ }^{*} p \leq 0.05$, $\S p \leq 0.01, \# p \leq 0.001$. $H I=$ hypoxia-ischemia, $S A L=$ saline, $M A P C S=$ multipotent adult progenitor cells 


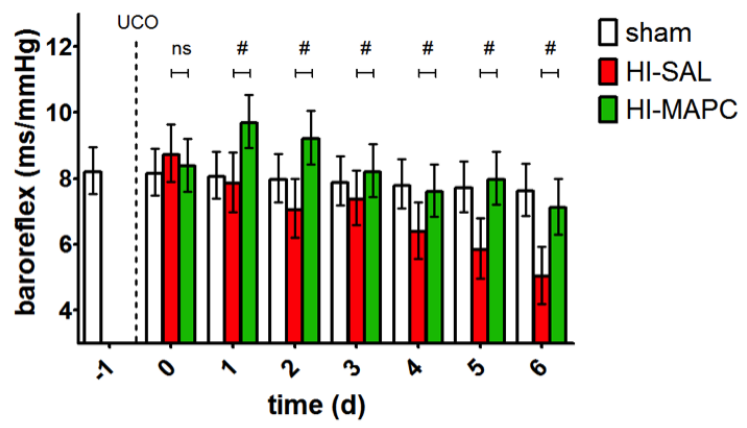

Figure 7.7: MAPCs prevented loss of baroreflex sensitivity. Global HI caused a significant gradual decline of baroreflex sensitivity over time, which was prevented by MAPCS treatment; means $\pm 95 \% \mathrm{Cl}$ and levels of significance of the treatment effect (HI-SAL vs. HI-MAPC) are depicted, which were calculated by the Bayesian multi-level model. There were no differences in baroreflex sensitivity between the sham-SAL and sham-MAPC groups. For readability purposes all sham treated animals (sham-SAL $n=8$ and shamMAPC $n=6$ ) were grouped and depicted as one sham group. HI-SAL $n=8, \mathrm{HI}$ MAPC $n=8 .{ }^{*} p \leq 0.05, \S p \leq 0.01, \# p \leq 0.001$. HI = hypoxia-ischemia, $S A L=$ saline, $M A P C=$ multipotent adult progenitor cells.

\section{Discussion}

Hypoxic-ischemic brain injury is common in preterm infants. To date no treatment options are available to reduce mortality and morbidity associated with brain injury in preterm infants. Our group has previously shown in a well-established pre-clinical model of global hypoxic-ischemia (HI) that mesenchymal stem cells (MSCs) are a promising candidate for the treatment of hypoxic-ischemic brain injury ${ }^{70}$. In the current study we investigated the neuroprotective capacity of multipotent adult progenitor cells (MAPCs), which are a subset of bone marrow-derived MSCs with more potent antiinflammatory and regenerative properties ${ }^{64-66,168-171}$. We showed that intravenous administration of MAPCs prevented functional and structural injury in the preterm brain and modulated the cerebral and peripheral inflammatory response after global $\mathrm{HI}$.

Our findings showed that MAPCs reduced seizure burden after global HI. This finding is clinically highly relevant, since several studies have shown that seizures in neonatal HIE are associated with adverse neurodevelopmental outcome ${ }^{178-180}$. In our model we detected seizure activity with amplitude-integrated EEG (aEEG), which is clinically used to monitor neonatal brain function and has a high sensitivity and specificity in predicting neurodevelopmental outcomes in neonates with $\mathrm{HI}$ brain injury ${ }^{181}$. Recorded seizure activity in our study was most likely located in the cortical layers. However, since the electrodes were placed directly on the dura, we may have recorded seizures or electrophysiological responses originating from deeper regions underlying the cortex (i.e. thalamus and hippocampus). In addition to cortical grey matter protection, the reduced seizure activity may therefore also represent protection of deeper brain structures. 
Besides reduction of seizure burden, we showed that MAPCs protected the baroreflex function. This is clinically of importance since the baroreflex is a vital part of the cardiovascular auto regulatory system in preterm infants, which secures adequate perfusion of the preterm brain ${ }^{175}$. The cerebrovascular system is still immature in prematurity making the preterm brain extremely vulnerable to fluctuations in blood pressure caused by $\mathrm{HI}$ or other adverse events such as infection ${ }^{182}$. Previously, we demonstrated that global HI disturbed normal development of the baroreflex, which resulted in impairment of heart rate mediated blood pressure control ${ }^{175}$. The fact that MAPC treatment improved baroreflex sensitivity indicated that the therapeutic effects reach as far as the highly conserved central brain functions in the brainstem and supports the concept of MAPC-induced protection against grey matter injury in the hypoxic-ischemic preterm brain. The protection of grey matter by MAPCs after global HI may be explained by findings of previous reports demonstrating that MAPCs improved neurogenesis and stimulated neurite outgrowth and synaptogenesis ${ }^{170,183,184}$.

Moreover, MAPCs have been previously shown to have potent anti-inflammatory properties in preclinical studies of traumatic and ischemic injury of the central nervous system ${ }^{64-66,170,171}$. Our study confirmed and extended these findings in a translational model of hypoxic-ischemic injury in the preterm brain by showing that MAPCs reduced the neuroinflammatory response in the subcortical white matter (SCWM), as evidenced by reduced microglial proliferation. We and others have shown that the microglial response plays a pivotal role in the etiology of hypoxic ischemic encephalopathy in the preterm brain ${ }^{29,185}$. We postulate that the observed MAPC-induced reduction of cerebral inflammation after global $\mathrm{HI}$ may be explained by two mechanisms; firstly, MAPCs have been shown to induce a microglial type switch from a pro-inflammatory M1 phenotype to an anti-inflammatory M2 phenotype, which is associated with reduced inflammation in the $\mathrm{CNS}^{65}$. Secondly, MAPCs may have reduced glutamate excitotoxicity in a similar fashion as MSCs, which were shown previously to inhibit glutamate receptor expression and function, thereby inhibiting microglial activation ${ }^{186}$. However, MAPCs did not attenuate the inflammatory response in the hippocampus. These spatial differences in anti-inflammatory effects are in line with previous data showing that the hippocampus is more severely affected by hypoxia-ischemia and therefore injury may be irreversible $\mathrm{e}^{29,69,70}$.

Besides attenuation of the cerebral inflammatory response, our findings indicated that MAPCs modulated the peripheral inflammatory response, which has been implicated in the etiology of hypoxic-ischemic injury in the preterm brain ${ }^{29,187-189}$. Our results showed that MAPCs prevented splenic involution. We have previously shown in the current model that splenic involution after global $\mathrm{HI}$ was caused by efflux of immune effector cells ${ }^{29}$. Furthermore, our findings demonstrated that intravenous administration of MSCs induced splenic T-cell tolerance ${ }^{70}$. The observed unresponsiveness of splenic T-cells was associated with reduced systemic mobilization of immune effector cells and concomitant reduction of brain infiltrating immune cells resulting in less severe inflammation in the preterm brain after global $\mathrm{HI}^{70}$. Based on these findings, we postulate that the MAPC-induced prevention of splenic involution indicated that the anti-inflammatory effect of MAPCs is mediated by the spleen. This concept is supported by previous findings showing a spleen-mediated neuroprotective effect of MAPCs in traumatic brain injury ${ }^{64}$. 
We could not reproduce the IL-10 mediated therapeutic effect of MAPCs observed in previous reports, which attributed the anti-inflammatory effect of MAPCs to an increase in splenic IL-10, inducing increased splenic and systemic numbers of protective regulatory T-cells in adult animals ${ }^{64}$. In our study splenic IL-10 mRNA levels were not altered by MAPCs in sham conditions. Instead, we found a marked increase of IL-10 mRNA under hypoxic-ischemic conditions, which was attenuated by administration of MAPCs. We postulate that the observed increase of splenic IL-10 mRNA after global HI is part of an endogenous regenerative response after injury. MAPCs may have reduced the injurious load eliciting such a response resulting in reduced IL-10 levels at the time of assessment ( 7 days after global $\mathrm{HI}$ ). This hypothesis is supported by previous reports showing activation of endogenous repair processes involving IL-10 mediated T-reg mobilization after cerebral ischemia ${ }^{187,188,190}$. Furthermore, early administration of MSCs was shown to prevent progression of hypoxic-ischemic injury ${ }^{70}$. These findings suggest that early administration of MAPCs prevents injury before endogenous repair mechanisms, such as IL-10 mediated T-reg mobilization, are activated.

White matter injury is the clinical hallmark of $\mathrm{HI}$ brain injury in the preterm brain ${ }^{3}$. Consistent with earlier studies ${ }^{29,69,70}$, we demonstrated that global hypoxia-ischemia induced marked hypomyelination of the preterm brain, which was prevented by intravenous administration of MAPCs. Microglial type switch and subsequent reduction of cerebral inflammation has been previously associated with remyelination ${ }^{191,192}$ and may therefore explain the observed reduction of white matter injury. Our study has several limitations. We did not perform time series of histologic analysis to assess temporal dynamics of global HI-induced injury and therapeutic effects of intravenous MAPC administration. Moreover we did not discriminate between the therapeutic effect between the first and second dose of MAPCs. These limitations are inherent to limited experimental groups to make large animal experiments feasible. Furthermore, no sheep-specific tools are currently available to analyze the microglial phenotypes, which did not allow studying the postulated microglial type-switch.

Our findings are clinically highly relevant for several reasons. First, we tested our hypothesis in a well established pre-clinical animal in which global HI was induced by transient umbilical cord occlusion accurately mimicking the common etiology of hypoxic-ischemic brain injury in preterm infants ${ }^{26,30}$. Second, this pre-clinical animal model allows for continuous in utero registration of relevant parameters (i.e. vital parameters and $\mathrm{aEEG}$ ) with strong clinical relevance and predictive value, which gives more insight in $\mathrm{HI}$-induced pathophysiology and efficacy of therapeutic options in a preclinical setting. Third, preclinical studies have confirmed safety and genomic stability of clinical grade MAPCs ${ }^{167,176}$ and clinical grade MAPC therapy is currently being tested in phase I/II clinical trials for the treatment of ischemic stroke, graft versus host disease (GVHD), acute myocardial infarction and inflammatory bowel disease ${ }^{169}$ (Jacobs, et al., 2013b), emphasizing clinical applicability of these cells.

\section{Conclusion}

In conclusion, we have shown in a pre-clinical animal model that MAPCs improve the functional and structural outcome of the preterm brain after global HI. Future studies should further establish the mechanism and long-term therapeutic effects of MAPC-induced neuroprotection in the developing preterm brain exposed to hypoxia- 
ischemia. Our study may form the basis for future clinical trials, which will evaluate whether MAPC therapy is capable of reducing neurological sequelae in preterm infants with hypoxic-ischemic brain injury. 


\section{Appendix A}

To facilitate analysis physiological data, parameter values from $192 \mathrm{~s}$ segments were grouped per 30 minutes by calculating a median value for each parameter, thereby creating time series for the various parameters at the individual level. Potential circadian rhythms were removed by notch filtering the time series at the frequency of 1 period/day. To avoid issues with pooling of data, we performed the statistical analysis of the time series using a Bayesian hierarchical model. Data pooling assigns the relative importance of variance at the individual level, i.e. within the subsequent measurements in each individual, and at the population level, i.e. between individual subjects. Many frequentist approaches to statistical time series analysis require an explicit approach to data pooling, which implies that a priori assumptions about variances are made. In a Bayesian framework these variances are modelled on the available data. This allows us to avoid this explicit choice, which is beneficial as we cannot make strong assumptions about the importance of variance at the individual and population levels.

Before performing the statistical analysis, measured values are shifted and scaled by subtraction of the overall mean and subsequent division by 2 standard deviations. The time series are then modelled at the individual, i.e. measurement, and population levels. At the measurement level we use the following model:

$$
\begin{aligned}
& y_{i}=\alpha_{j k[i]}+\beta t_{i}+\epsilon_{i} \\
& \epsilon_{i} \sim t\left(5,0, \sigma_{y}\right)
\end{aligned}
$$

Each measured value $y_{i}$, with $i=1, \ldots, n$ and $n$ the total number of measurements, is composed of a non-stochastic part, $\alpha_{j k[i]}+\beta t_{i}$ and a stochastic part $\epsilon_{i}$. The stochastic part represents deviations at the measurement level due to natural variations and unknown errors, and is modelled with a $t$-distribution with 5 degrees of freedom, mean 0 , and scaling factor $\sigma_{y}$ positive and smaller than $10^{193}$. In the sham-SAL group we assume that changes over time can be adequately approached using a linear model that directly captures time, with slope $\beta$ and time $t_{i}$, where $t=0$ coincides with the moment of occlusion. However, the linearity assumption does not hold for the $\mathrm{HI}$ intervention groups, and we capture the a priori unknown non-linear behaviour by modelling $\alpha_{j k}$, for individuals $j=1, \ldots, m$, with $m$ the number of individual subjects, in time windows $k=$ baseline $, 0,1, \ldots, 6$ :

$$
\begin{aligned}
& \alpha_{j k}=a_{0}+g_{U C O, k} u_{U C O, j k}+g_{\text {MAPC }, k} u_{M A P C, j k}+g_{U C O+M A P C, k} u_{U C O, j k} u_{M A P C, j k}+\epsilon_{j} \\
& \epsilon_{j} \sim t\left(5,0, \sigma_{\alpha}\right)
\end{aligned}
$$

$\alpha_{j k}$ is, like $y_{i}$ composed of a non-stochastic and a stochastic part. The stochastic part $\epsilon_{j}$ captures unexplained variance between individuals. $g_{U C O, k}, g_{M A P C, k}$ and $g_{U C O+M A P C, k}$ are the population effects of the UCO intervention, the MAPC treatment and their interaction respectively. $u_{U C O, j k}$ and $u_{M A P C, j k}$ are contrasts, i.e. 0 if the subject respectively had a sham occlusion or saline treatment, or during the baseline period, and 1 otherwise. $a_{0}$ is the population mean in the absence of intervention or treatment effects. 
The Bayesian model was assessed using four Monte Carlo chains of 2500 iterations, including 500 warm-up iterations, per chain. Proper convergence and mixing of chains was checked. Resulting model data was subsequently rescaled to the original scale, and used for inference. 

8 General discussion 

The personal and societal burden of hypoxic-ischemic encephalopathy is immense. Neonatal encephalopathy globally leads to 50 million disability-adjusted life-years $(\mathrm{DALY})^{4}$. In comparison, the entire spectrum of cancer was estimated to lead to 169 million DALY globally ${ }^{5}$. Though part of the burden can be reduced through general improvements to obstetric care in regions where public health care is less developed, additional reduction can only be realised through new and innovative improvements in perinatal care. In this context, more accurate and earlier detection of conditions leading to HIE are essential to start specific treatment regimes at the earliest moment to prevent or limit the extent of brain injury. Treatment options should furthermore improve through novel neuroprotective and/or neuroregenerative treatments for HIE, especially for preterm infants, for whom no treatment is currently available. However, before any treatment can start, infants require assessment of HIE severity and progress to create a treatment prognosis. Markers to reliably base this outcome prognosis on are still absent, or only effective once the subacute phase of brain injury has started ${ }^{12}$. Furthermore, neuronal injury may also be assessed at the functional level. Monitoring aspects of cortical, subcortical and autonomic functions may help identify conditions that lead to additional risk to the infant's well-being.

In this thesis we examined currently available and new markers for computer algorithms for the early detection of hypoxia-ischemia and for the assessment of cortical, subcortical and autonomic function. We will briefly discuss the findings from the previous chapters and place them into the broader context of data-driven algorithms for clinical decision support systems.

\section{Early detection of hypoxia-ischemia}

Currently used clinical methods to detect fetal conditions that lead to HIE are ineffective and do not prevent HIE-related outcomes in clinical settings $s^{31,35,36}$. ST waveform analysis was implemented in a clinical device with the aim of warning for conditions of hypoxia and acidosis. The ST segment start after the QRS complex and ends at the top of the T wave. This ST segment changes in response to hypoxia and acidosis $^{32-34}$. However, both the implementation of STAN in the clinical device and the implementation of the device alarm algorithm within a computer algorithm failed to assess severely hypoxic conditions in our preterm model for severe hypoxia-ischemia. We speculate that these findings resulted from limitations of the device alarm algorithm, which interprets the T/QRS marker to assess hypoxia. T/QRS is the proportion of the T wave amplitude with respect to the QRS complex in the ECG. The device alarm algorithm assumes the response of the ST segment to hypoxia to produce a rise in $T / Q R S$ values. We found this was often not the case as in several subjects T/QRS values became more negative in response to hypoxia and acidosis.

We showed that ECG waveform-based markers are less useful for detecting hypoxic and acidotic conditions in the ovine fetus. The shape of fetal ECG waveforms depends on the orientation of the measurement lead with respect to the electrical heart vector. Changes in orientation thus affect the shape of the ECG waveform. Hence inherent problems exist in assessing the ST segment in the ECG recorded using a single unipolar scalp electrode during birth. Differences in cardiac anatomy and differences in myocardial response to hypoxia and acidosis between individuals, and variation of scalp electrode orientation with regards to the heart vector during birth within the same 
individual, lead to changes in ST waveform. These changes in ST waveform may hamper ST waveform analysis. Reconstruction of a three dimensional fetal heart vector could increase the reliability of waveform based markers. A three dimensional heart vector allows direct assessment of hypoxia-induced changes in the heart vector, instead of being limited to a projection of the heart vector when measuring with a scalp electrode. Investigation of techniques for non-invasive multi-lead measurement of fetal ECG are under way ${ }^{194-196}$.

Interval-based markers performed better in detecting hypoxia within the fetal ovine model than waveform-based ECG markers. Notably, we could confirm the findings of Oudijk et al. with regards to QT interval shortening as a marker for hypoxia ${ }^{41}$. The QT interval reflects ventricular contraction and repolarisation. Oxidative metabolism is halted during hypoxia. Anaerobic glycolysis is maintained until blood acidity from lactate production becomes too low and glycolysis is attenuated ${ }^{197}$. The switch to anaerobic glycolysis is accompanied by a surge in epinephrine ${ }^{91}$. Release of epinephrine was linked to QT interval shortening ${ }^{92,93}$, which may have led to our finding.

\section{Assessment of cortical function: detection of short seizures for seizure detection algorithms}

Seizure activity occurs concomittantly with neuronal losses, and have been implicated in exarcabating the brain injury suffered by the infant. Physicians can prescribe medication to suppress seizures. Detection of seizure activity could both alarm physicians to the need for closer observation and could be used to assess the severity of neuronal loss in HIE, without using MRI. In preterm infants seizures are often short ( $<1$ minute) and more likely to be missed by automatic detection algorithms. We tested a method to increase performance of seizure detection algorithms for the detection of such short seizures. Current seizure detection algorithms assess the relatively stable part of the seizure. However, transitions to and from seizure state take up a significant portion of total seizure duration for short seizures. As a consequence, the stable part of the seizure is short or absent in short seizures, hampering detection performance of current algorithms. The proposed method significantly improved detection performance by capturing information contained in the transition from and to seizure state. Furthermore since the method produces a new input feature, it can be easily implemented in current and future algorithms.

The detection algorithm was used to assess bipolar EEG. While often used for clinical monitoring, bipolar EEG has the drawback of less available EEG channels than the reduced $10 / 20$ system $^{126}$. The reduced availability of EEG channels was inferred to be an important reason for lower detection performance of our detection algorithm compared to that reported by Temko et al. ${ }^{49}$ The reduced $10 / 20$ system improves electrode coverage and introduces redundancy with respect to bipolar EEG. The reduced 10/20 system allows detection of seizures that are distant from the bipolar EEG leads, because the effect of reduction in signal amplitude due to distance is attenuated through increased electrode coverage ${ }^{118}$. Redundancy allows artefact detection and correction which reduces the number of false detections ${ }^{120}$. We expect that our method will lead to improved detection performance when trained and applied in recordings from the reduced 10/20 system. However, though more seizures can be detected using 
the reduced 10/20 lead system, bipolar EEG recordings have the advantage of requiring less patient handling and in being more consistently interpretable ${ }^{198}$.

Assessment of subcortical function: interhemispheric burst synchrony as a potential marker for neuronal loss

We translated principles for manually assessing burst synchrony in the $\mathrm{EEG}^{56,59,123}$ into a computer algorithm. The algorithm was used to assess burst synchrony in preterm infants of 28 to 35 weeks post-menstrual age in a consistent manner. This was required since inconsistencies between Lombroso and Anderson et al. might have led to their different conclusions with regards to maturational effects on burst synchrony. Investigating maturational changes in interhemispheric burst synchrony was necessary to evaluate how useful this marker could be for assessment of subcortical function in infants with (suspected) HIE. Changes in burst synchrony due to maturation and changes in burst synchrony due to neuronal loss might interfere or compensate each another.

Using a computerized algorithm, we demonstrated a high degree of interhemispheric burst synchrony, irrespective of post-menstrual age. Synchrony of EEG bursts is facilitated by long-distance connections which link homologous areas of both hemispheres ${ }^{53,54}$. Such long-distance connections are both cortico-cortical (such as the corpus callosum) and thalamocortical connections. These connections are formed during the second half of gestation ${ }^{199-201}$ and have become functional by the start of our analysis period at 28 weeks ${ }^{200,202}$. Thus lack of maturational effect on burst synchrony might be caused by the fact that the connections to facilitate synchrony have already developed. Based on the findings of Tharpe et al. ${ }^{55}$ we hypothesize that severe hypoxiaischemia should significantly affect interhemispheric burst synchrony. Interhemispheric burst synchrony should therefore be evaluated as a potential marker for assessing HIE severity. The marker could be particularly useful for preterm infants where other markers for HIE severity are often difficult to establish ${ }^{14-17}$. An initial step could be testing the marker within the ovine fetal model for severe hypoxia-ischemia in which interhemispheric burst synchrony is expected to significantly decrease. This will be a challenge because of current technical limitations in implementing a reduced 10/20 lead placement for EEG in the ovine fetal model. Afterwards, or as an alternative, interhemispheric burst synchrony should be evaluated in EEG recordings recorded in preterm infants with HIE.

Our implementation of interhemispheric burst synchrony as a marker of functional connectivity is a direct translation from observational guidelines to a computer algorithm. Other EEG-based markers for functional connectivity have also been defined. Meijer et al. assessed magnitude coherence as marker for functional connectivity in healthy preterm infants ${ }^{203}$. This marker is based on calculations in the frequency domain. Koolen et al. devised a marker for connectivity which was likewise based on the frequency domain ${ }^{204}$. Koolen et al. also assessed a promising marker for interhemispheric synchrony devised by Räsänen et al. ${ }^{205,206}$, which was based on testing statistical independence in bipolar EEG channels. An advantage of the latter marker is that it does not require reduced 10/20 for assessment. The markers mentioned above cover a different aspect of EEG signal than the purely time-domain based marker we 
assessed, and might contain complementary information concerning an infant's subcortical function.

Assessment of autonomic function: monitoring fetal cardiovascular autonomic regulation during severe HIE

The baroreceptor reflex mechanism buffers short term changes in blood pressure through various pathways, among which are changes in heart rate. Heart rate can be readily assessed from fetal ECG, and hence we assessed how baroreceptor reflex heart rate mediated regulation of blood pressure is affected by severe HIE. In our standardised fetal ovine model it became apparent that barorecepter reflex sensitivity decreased significantly over the course of several days. In the subsequent stem cell treatment study we found a comparable trend within the asphyxiated animals. Two questions remain unanswered however: 1) Does an impaired baroreceptor reflex after HIE increase neuronal loss? 2) What is the mechanistic background for the effect of HIE on baroreceptor reflex function?

First, whether an impaired baroreceptor reflex after HIE increases neuronal loss cannot be directly answered by current research. Indirect evidence points to this being the case. Liu et al. demonstrated that disruption of the baroreceptor reflex resulted in increased neuronal loss after unilateral cerebral hypoxia-ischemia ${ }^{207}$. We have demonstrated that severe global hypoxic-ischemia attenuated baroreceptor reflex function. Neonatal hypotension and hypertension are harmful to the neonatal cerebrovasculature ${ }^{62}$ and both conditions can result from impaired baroreceptor reflex function. We also demonstrated that stem cell treatment reduced white matter loss after severe hypoxia-ischemia and that baroreceptor reflex function was retained. Preservation of baroreceptor reflex may thus be a pathway for neuronal protection. To demonstrate the protective role of the baroreceptor reflex in HIE, it is necessary to directly regulate baroreceptor reflex. Devices for direct baroreceptor regulation are being evaluated as a treatment for hypertension ${ }^{208}$. However, if HIE causes functional baroreceptor loss through injury to brain stem nuclei, stimulating baroreceptors will be ineffective and direct stimulation of the cardiac branches of the vagal nerve is necessary. An alternative method to test the hypothesis is through correlating baroreceptor sensitivity and white matter loss. However the disadvantage of such a test is that the time period over which the attenuated baroreceptor reflex function effects the hypothesised additional neuronal loss is not known.

Though we were not able to determine by what mechanism severe hypoxiaischemia affected baroreceptor function, we hypothesize that severe hypoxia-ischemia causes neuronal loss within the nuclei of the brain stem which are responsible for the baroreceptor reflex. Severe hypoxia-ischemia is known to cause neuronal loss in the brain stem ${ }^{87}$, though it is unclear which nuclei are affected to what degree. It is plausible, but unconfirmed, that stem cell treatment protects brain stem nuclei from injury. This hypothesis would explain why baroreceptor reflex was not attenuated after treatment of severe HIE with stem cells.

Determining baroreceptor reflex function in infants with HIE is important for two reasons. The first reason is that an attenuated reflex mechanism could predispose the infant to developing more neuronal damage by dysregulated perfusion and 
hypertension. The second reason is that stem cell treatment protects baroreceptor reflex function, and could eventually be used clinically in preterm infants with HIE.

The preterm ovine model for severe hypoxic-ischemic encephalopathy

Because hypoxia in clinical settings is usually gradual and periodic, our animal model does not reflect the most common clinical course of hypoxia. Since in our model an umbilical cord occlusion leads to severe hypoxia-ischemia within minutes, the model is useful for examining which markers might be effective in detecting hypoxic conditions. However, but it should be noted that performance of markers within the animal model may not reflect clinical effectiveness. Promising markers will need to be evaluated using clinical recordings. At the same time, markers that do not perform well in the model, such as waveform-based baseline and episodic T/QRS rise markers that were used to generate alarms by the STAN device, are unlikely to perform well in clinical settings due to lack of sensitivity and positive predictive value.

From bench to bedside-developing clinical decision support systems

Perinatal clinical care can be improved through the use of well-designed clinical decision support systems. Development of such systems ought to take place in roughly three stages. The first stage, with which most of this thesis is concerned, is the conceptual stage. The aim of the conceptual stage is to formulate and subsequently assess whether markers, methods and algorithms are potentially useful in clinical cases. A controlled testing environment, such as that offered by animal models, is important in this stage.

The second stage is the pre-clinical stage. Algorithms, methods and markers in the pre-clinical stage are evaluated by using either animal models that closely reflect clinical reality, or data recorded in human subjects. Naturally there is some overlap with the first stage, as the same data might be used.

The third stage is the clinical stage. A prototype decision support system is devised by introducing markers, methods and algorithms that have been found relevant in the earlier stages into a clinical platform. Clinicians are trained to interpret the information provided by the decision support system and a randomised control trial is set up to assess the performance in clinical practice.

The STAN device would have benefited if implementation of ST waveform analysis in the device would have been checked against data from pre-clinical and conceptual studies. Limitations of the actual clinical implementation, as demonstrated in chapter two, might thus have come to light before many hospitals used public money to purchase an apparatus which implemented a method that has no apparent clinical benefit ${ }^{35,36}$.

\section{Considerations for developing monitoring algorithms}

Current clinical monitoring algorithms are often constructed using manually set threshold criteria. These threshold criteria might be subjective, arbitrary, or specific to small number of situations, which hampers algorithm performance. The STAN device algorithm is one example that I evaluated in this thesis. The threshold rules for STAN- 
device baseline and episodic T/QRS rise alarms, as well as the length of time windows seem based on arbitrary choices. Why would a baseline T/QRS rise of 0.050 be significant and produce an alarm, and 0.049 not? Why would a T/QRS value be checked against the median T/QRS value of the previous 10 minutes instead of 5 or 15 minutes?

Machine learning techniques can be used to reduce biases introduced by manually constructed sets of rules. Machine learning uses features from recorded signals to train a rule set, or classifier. Many different machine learning techniques exist, see e.g. P. Domingo ${ }^{209}$ for examples. Several examples of machine learning techniques were used throughout this thesis. In the third chapter we used measured data to set threshold values for hypoxia alarms. In the fourth chapter we used a support vector machine to identify seizures in EEG recordings, and in the seventh chapter we used Bayesian regression to estimate differences between treatment groups.

Machine learning techniques can be used to combine information contained in markers and use them as input features. Combining features can lead to better predictions for clinical algorithms, for example in seizure detection ${ }^{101,210}$ and automated EEG burst detection ${ }^{127}$. A combination of features was used by a support vector machine to detect seizure activity in the fourth chapter. Of the other machine learning techniques in this thesis, Bayesian regression is useful for combining features. Threshold techniques are often not useful for this purpose, as interaction between features cannot be captured using this technique.

Machine learning for clinical algorithms is usually conducted in two stages using a leave-one-out, bootstrap or cross-validation method ${ }^{106}$. These stages are classifier training and validation on a test set. Post-processing after classifier training has consequences for predictive capacity of the algorithms, but does not fit directly in the above stages. One choice would be to prescribe post-processing parameters manually. However this should be avoided if possible, to prevent subjective biases. Another choice is to base post-processing parameter values on either the training or validation data set. However, this again has potential to introduce biases. To avoid biases we introduced a stage for optimising post-processing parameters that was separate from classifier training and validation stages. As a consequence, we adapted the cross-validation method to use three instead of two stages, as was described in chapter four.

Chapter four also discussed another issue in algorithm training. How does one define 'optimal' performance? In the example of seizure detection, 'optimal' might be defined as detecting all seizures in the EEG recording, or as detecting no false alarms. Both are goals which are often not attainable without severe drawbacks. By aiming to detect all seizures, many false alarms will be introduced. In clinical practice this leads to alarm fatigue ${ }^{72}$. Alarm fatigue leads to clinicians not identifying some clinically relevant alarms. On the other hand, eliminating false alarms can cause many clinically relevant alarms to be missed as well. A trade-off between complete detection and no false alarms is therefore necessary. More importantly, it is obvious that the definition used for 'optimal' performance directly affects what performance is reported and reflects the choice made by the investigators. In this thesis we have clearly stated what performance was assumed to be 'optimal', which depends on the purpose of the study.

Irrespective of the machine learning method, it would be preferable to train and test algorithms using clinical data. The fetal ovine model, as has been mentioned, represents 
an extreme course of hypoxia and acidosis that is not commonly found in clinical practice. Using the current recordings to construct classifiers for early detection of hypoxia and acidosis is unlikely to produce satisfactory results when used clinically, as the classifier will be very specific to the problem of severe hypoxia-ischemia. To properly train a classifier using machine learning requires that the true condition is known. For example, in chapter four it was necessary to define seizures in the EEG to properly train the support vector machine classifier. For the problem of early detection of hypoxia and acidosis, intermittent measurements of blood acidity and arterial blood oxygen levels are necessary to assess the 'true' condition of the fetus with regards to hypoxia and acidosis. Clinical recordings used for training a classifier for early detection of hypoxia and acidosis thus not only requires the physiological signal used to define markers on, but also intermittent blood gas measurements.

\section{Conclusion}

Computer-assisted monitoring technology is playing an increasingly important role in perinatal care. Early assessment of fetal hypoxia is a prime target for the development of clinical decision support systems. Machine learning offers many tools in the construction of suitable algorithms for these decision support systems. Such algorithms should be tested and evaluated at every point of development from input feature to algorithm performance. Several features and methods were tested and evaluated for future implementation in decision support systems. A number of ECGbased markers for hypoxia were compared during controlled umbilical cord occlusion in the fetal ovine model. Of these markers we found that the length of ventricular activation and repolarisation time, and heart rate may be useful interval-based markers for assessing fetal hypoxia. In contrast, waveform analysis of the ST segment was not useful as early marker of fetal hypoxia. When compared to interval-based ECG markers, waveform-based markers are less reliable due to dependency on ECG lead and heart vector orientation. Furthermore, we developed and evaluated a method that facilitated detection of short seizures in preterm infants. In addition, an automated algorithm to assess interhemispheric burst synchrony was evaluated as a marker for (sub)cortical function. We demonstrated that, irrespective of postmenstrual age, healthy infants showed a high degree of interhemispheric burst synchrony. This finding indicates the early fetal presence of cortico-cortical (e.g. corpus callosum) and thalamocortical connections. This justifies further investigation of interhemispheric burst synchrony during conditions of neonatal HIE. The baroreceptor reflex associated cardiovascular fluctuations were assessed as marker for autonomic function. HIE clearly attenuated operation of the baroreceptor reflex but this function was preserved after multi-stem cell treatment. This illustrates the potential of a clinically relevant read-out signal marker to identify treatment effects of HIE. In addition, this thesis illustrates the success of a multidisciplinary approach that involves clinicians, (clinical) physicists and technicians in the development and assessment of computerized monitoring techniques for challenging clinical problems. 

References 

1. Kurinczuk, J. J., White-Koning, M. \& Badawi, N. Epidemiology of neonatal encephalopathy and hypoxicischaemic encephalopathy. Early Hum Dev 86, 329-338 (2010).

2. Sarnat, H. B. \& Sarnat, M. S. Neonatal encephalopathy following fetal distress. A clinical and electroencephalographic study. Arch Neuro/ 33, 696-705 (1976).

3. Volpe, J. J. Brain injury in premature infants: a complex amalgam of destructive and developmental disturbances. Lancet Neurol 8, 110-124 (2009).

4. Lee, A. C. C. et al. Intrapartum-related neonatal encephalopathy incidence and impairment at regional and global levels for 2010 with trends from 1990. Pediatr Res 74 Suppl 1, 50-72 (2013).

5. Soerjomataram, I. et al. Global burden of cancer in 2008: A systematic analysis of disability-adjusted lifeyears in 12 world regions. Lancet 380, 1840-1850 (2012).

6. Gunn, A. J. \& Bennet, L. Timing of injury in the fetus and neonate. Curr Opin Obstet Gynecol 20, 175-181 (2008).

7. Drury, P. P., Bennet, L. \& Gunn, A. J. Mechanisms of hypothermic neuroprotection. Semin Fetal Neonatal Med 15, 287-292 (2010).

8. Yenari, M. A. \& Han, H. S. Neuroprotective mechanisms of hypothermia in brain ischaemia. Nature Reviews Neuroscience 13, 267-278 (2012).

9. Thoresen, M. Who should we cool after perinatal asphyxia? Semin Fetal Neonatal Med 20, 66-71 (2015).

10. Jacobs, S. E., Hunt, R., Tarnow-Mordi, W. O., Inder, T. E. \& Davis, P. G. Cooling for newborns with hypoxic ischaemic encephalopathy. Cochrane Database Syst Rev 1, CD003311-CD003311 (2012).

11. Azzopardi, D. V. et al. Effects of Hypothermia for Perinatal Asphyxia on Childhood Outcomes. N Engl J Med 371, 140-149 (2014).

12. Bennet, L., Booth, L. C. \& Gunn, A. J. Potential biomarkers for hypoxic-ischemic encephalopathy. Semin Fetal Neonatal Med 15, 253-260 (2010).

13. Iwata, O. et al. 'Therapeutic time window' duration decreases with increasing severity of cerebral hypoxiaischaemia under normothermia and delayed hypothermia in newborn piglets. Brain Res 1154, 173180 (2007).

14. Forslund, M. \& Bjerre, I. Neurological assessment of preterm infants at term conceptional age in comparison with normal full-term infants. Early Hum Dev 8, 195-208 (1983).

15. Hegyi, T. et al. The apgar score and its components in the preterm infant. Pediatrics 101, 77-81 (1998).

16. Toet, M. C. \& Lemmers, P. M. A. Brain monitoring in neonates. Early Hum Dev 85, 77-84 (2009).

17. André, M. et al. Electroencephalography in premature and full-term infants. Developmental features and glossary. Neurophysiol Clin 40, 59-124 (2010).

18. Murray, D. M. et al. Defining the gap between electrographic seizure burden, clinical expression and staff recognition of neonatal seizures. Arch Dis Child Fetal Neonatal Ed 93, F187-F191 (2008).

19. Wilson, S. B., Scheuer, M. L., Plummer, C., Young, B. \& Pacia, S. Seizure detection: Correlation of human experts. Clin Neurophysiol 114, 2156-2164 (2003).

20. Boylan, G. B., Stevenson, N. J. \& Vanhatalo, S. Monitoring neonatal seizures. Semin Fetal Neonatal Med 18, 202-208 (2013).

21. Faul, S., Boylan, G. B., Connolly, S., Marnane, W. P. \& Lightbody, G. An evaluation of automated neonatal seizure detection methods. Clin Neurophysiol 116, 1533-1541 (2005).

22. Bellazzi, R. \& Zupan, B. Predictive data mining in clinical medicine: Current issues and guidelines. Int J Med Inform 77, 81-97 (2008).

23. Vuguin, P. M. Animal models for small for gestational age and fetal programming of adult disease. Horm Res 68, 113-123 (2007). 
24. Kramer, B. W., Kallapur, S., Newnham, J. \& Jobe, A. H. Prenatal inflammation and lung development. Semin Fetal Neonatal Med 14, 2-7 (2009).

25. Bennet, L. \& Gunn, A. J. The fetal heart rate response to hypoxia: insights from animal models. Clin Perinatol 36, 655-672 (2009).

26. Gunn, A. J. \& Bennet, L. Fetal hypoxia insults and patterns of brain injury: insights from animal models. Clin Perinatol 36, 579-593 (2009).

27. Yager, J. Y. \& Ashwal, S. Animal Models of Perinatal Hypoxic-Ischemic Brain Damage. Pediatr Neurol 40, 156-167 (2009).

28. Gantert, M. et al. Chorioamnionitis: a multiorgan disease of the fetus? J Perinatol 30 Suppl, S21-S30 (2010).

29. Jellema, R. K. et al. Cerebral inflammation and mobilization of the peripheral immune system following global hypoxia-ischemia in preterm sheep. J Neuroinflammation 10, 13 (2013).

30. Back, S. A., Riddle, A., Dean, J. M. \& Hohimer, A. R. The instrumented fetal sheep as a model of cerebral white matter injury in the premature infant. Neurotherapeutics 9, 359-370 (2012).

31. Neilson, J. P. Fetal electrocardiogram (ECG) for fetal monitoring during labour. Cochrane Database Syst Rev 3, CD000116 (2013).

32. Rosén, K. G. \& Kjellmer, I. Changes in the fetal heart rate and ECG during hypoxia. Acta Physiol Scand 93, 59-66 (1975).

33. Greene, K. R., Dawes, G. S., Lilja, H. \& Rosén, K. G. Changes in the ST waveform of the fetal lamb electrocardiogram with hypoxemia. Am J Obstet Gynecol 144, 950-958 (1982).

34. Rosén, K. G., Amer-Wåhlin, I., Luzietti, R. \& Norén, H. Fetal ECG waveform analysis. Best Pract Res Clin Obstet Gynaecol 18, 485-514 (2004).

35. Alfirevic, Z., Devane, D. \& Gyte, G. M. L. Continuous cardiotocography (CTG) as a form of electronic fetal monitoring (EFM) for fetal assessment during labour. Cochrane Libr 3, CD006066 (2013).

36. Salmelin, A. et al. Fetal monitoring with computerized ST analysis during labor: a systematic review and meta-analysis. Acta Obstet Gynecol Scand 92, 28-39 (2013).

37. Widmark, C., Lindecrantz, K., Murray, H. \& Rosén, K. G. Changes in the PR, RR intervals and ST waveform of the fetal lamb electrocardiogram with acute hypoxemia. J Dev Physiol 18, 99-103 (1992).

38. Westgate, J. A. et al. Do fetal electrocardiogram PR-RR changes reflect progressive asphyxia after repeated umbilical cord occlusion in fetal sheep? Pediatr Res 44, 297-303 (1998).

39. Van Wijngaarden, W. J. et al. Changes in the PR interval-fetal heart rate relationship of the electrocardiogram during fetal compromise in chronically instrumented sheep. Am J Obstet Gynecol 175, 548-554 (1996).

40. Keunen, H., Van Wijngaarden, W. J., Sahota, D. S. \& Hasaart, T. H. M. The PR interval-fetal heart rate relationship during repetitive umbilical cord occlusions in immature fetal sheep. Eur J Obstet Gynecol Reprod Biol 89, 69-74 (2000).

41. Oudijk, M. A. et al. The effects of intrapartum hypoxia on the fetal QT interval. BJOG 111, 656-660 (2004).

42. Ronen, G. M., Buckley, D., Penney, S. \& Streiner, D. L. Long-term prognosis in children with neonatal seizures: A population-based study. Neurology 69, 1816-1822 (2007).

43. Thibeault-Eybalin, M. P., Lortie, A. \& Carmant, L. Neonatal Seizures: Do They Damage the Brain? Pediatr Neurol 40, 175-180 (2009).

44. Scher, M. S. Neurophysiological assessment of brain function and maturation: I. A measure of brain adaptation in high risk infants. Pediatr Neurol 16, 191-198 (1997).

45. Cherian, P. J. et al. Validation of a new automated neonatal seizure detection system: a clinician's perspective. Clin Neurophysiol 122, 1490-1499 (2011). 
46. Liu, A., Hahn, J. S., Heldt, G. P. \& Coen, R. W. Detection of neonatal seizures through computerized EEG analysis. Electroencephalogr Clin Neurophysiol 82, 30-37 (1992).

47. Gotman, J., Flanagan, D., Rosenblatt, B., Bye, A. \& Mizrahi, E. M. Evaluation of an automatic seizure detection method for the newborn EEG. Electroencephalogr Clin Neurophysiol 103, 363-369 (1997).

48. Celka, P. \& Colditz, P. B. A computer-aided detection of EEG seizures in infants: A singular-spectrum approach and performance comparison. IEEE Trans Biomed Eng 49, 455-462 (2002).

49. Temko, A., Thomas, E. M., Marnane, W. P., Lightbody, G. \& Boylan, G. B. EEG-based neonatal seizure detection with Support Vector Machines. Clin Neurophysiol 122, 464-473 (2011).

50. Aarabi, A., Grebe, R. \& Wallois, F. A multistage knowledge-based system for EEG seizure detection in newborn infants. Clin Neurophysiol 118, 2781-2797 (2007).

51. Aarabi, A., Fazel-Rezai, R. \& Aghakhani, Y. A fuzzy rule-based system for epileptic seizure detection in intracranial EEG. Clin Neurophysiol 120, 1648-1657 (2009).

52. Mitra, J. et al. A multistage system for the automated detection of epileptic seizures in neonatal electroencephalography. J Clin Neurophysiol 26, 218-226 (2009).

53. Jones, E. G. Synchrony in the interconnected circuitry of the thalamus and cerebral cortex. Ann N Y Acad Sci 1157, 10-23 (2009).

54. Fingelkurts, A. A., Fingelkurts, A. A. \& Kähkönen, S. Functional connectivity in the brain--is it an elusive concept? Neurosci Biobehav Rev 28, 827-836 (2005).

55. Tharp, B. R., Cukier, F. \& Monod, N. The prognostic value of the electroencephalogram in premature infants. Electroencephalogr Clin Neurophysiol 51, 219-236 (1981).

56. Lombroso, C. T. Neonatal polygraphy in full-term and premature infants: a review of normal and abnormal findings. J Clin Neurophysiol 2, 105-156 (1985).

57. Korotchikova, I., Stevenson, N. J., Walsh, B. H., Murray, D. M. \& Boylan, G. B. Quantitative EEG analysis in neonatal hypoxic ischaemic encephalopathy. Clin Neurophysiol 122, 1671-1678 (2011).

58. Lynch, N. E. et al. The temporal evolution of electrographic seizure burden in neonatal hypoxic ischemic encephalopathy. Epilepsia 53, 549-557 (2012).

59. Anderson, C. M., Torres, F. \& Farao, A. The EEG of the early premature. Electroencephalogr Clin Neurophysiol 60, 95-105 (1985).

60. Sykora, M. et al. Impaired baroreflex sensitivity predicts outcome of acute intracerebral hemorrhage. Crit Care Med 36, 3074-3079 (2008).

61. Sykora, M., Diedler, J., Turcani, P., Hacke, W. \& Steiner, T. Baroreflex: A new therapeutic target in human stroke? Stroke 40, (2009).

62. Perlman, J. M. The relationship between systemic hemodynamic perturbations and periventricularintraventricular hemorrhage--a historical perspective. Semin Pediatr Neurol 16, 191-199 (2009).

63. Locatelli, F. et al. Stem cell therapy in stroke. Cellular and Molecular Life Sciences 66, 757-772 (2009).

64. Walker, P. A. et al. Intravenous multipotent adult progenitor cell therapy for traumatic brain injury: Preserving the blood brain barrier via an interaction with splenocytes. Exp Neurol 225, 341-352 (2010).

65. Walker, P. A. et al. Intravenous multipotent adult progenitor cell therapy after traumatic brain injury: modulation of the resident microglia population. J Neuroinflammation 9, 228 (2012).

66. Yasuhara, T. et al. Intravenous grafts recapitulate the neurorestoration afforded by intracerebrally delivered multipotent adult progenitor cells in neonatal hypoxic-ischemic rats. J Cereb blood flow Metab 28, 1804-1810 (2008).

67. Rosén, K. G. \& Isaksson, O. Alterations in fetal heart rate and ECG correlated to glycogen, creatine phosphate and ATP levels during graded hypoxia. Neonatology 30, 17-24 (1976). 
68. Amer-Wåhlin, I., Yli, B. \& Arulkumaran, S. Foetal ECG and STAN technology-a review. Eur Clin Obstet Gynaecol 1, 61-73 (2005).

69. Jellema, R. K. et al. Systemic G-CSF attenuates cerebral inflammation and hypomyelination but does not reduce seizure burden in preterm sheep exposed to global hypoxia-ischemia. Exp Neurol 250, 293303 (2013).

70. Jellema, R. K. et al. Mesenchymal stem cells induce T-cell tolerance and protect the preterm brain after global hypoxia-ischemia. PLoS One 8, e73031 (2013).

71. Taylor, R. Interpretation of the correlation coefficient: a basic review. J Diagn Med Sonogr 6, 35-39 (1990).

72. Cvach, M. Monitor Alarm Fatigue : An Integrative Review. Biomed Instrum Technol 46, 268-277 (2012).

73. Welin, A.-K. et al. Electrocardiographic changes following umbilical cord occlusion in the midgestation fetal sheep. Acta Obstet Gynecol Scand 84, 122-128 (2005).

74. Wassink, G. et al. Does Maturity Affect Cephalic Perfusion and T/QRS Ratio during Prolonged Umbilical Cord Occlusion in Fetal Sheep? Obstet Gynecol Int 2014, 314159 (2014).

75. Larma, J. D. et al. Intrapartum electronic fetal heart rate monitoring and the identification of metabolic acidosis and hypoxic-ischemic encephalopathy. Am J Obstet Gynecol 197, 301.e1-301.e8 (2007).

76. Belfort, M. A. \& Saade, G. R. ST segment analysis as an adjunct to electronic fetal monitoring, Part I: background, physiology, and interpretation. Clin Perinatol 38, 143-157 (2011).

77. Zhang, D. Wavelet Approach for ECG Baseline Wander Correction and Noise Reduction. Conf Proc IEEE Eng Med Biol Soc 2, 1212-1215 (2005).

78. Pan, J. \& Tompkins, W. J. A real-time QRS detection algorithm. IEEE Trans Biomed Eng 32, 230-236 (1985).

79. Boucheham, B., Ferdi, Y. \& Batouche, M. C. Piecewise linear correction of ECG baseline wander: A curve simplification approach. Comput Methods Programs Biomed 78, 1-10 (2005).

80. Malik, M. et al. Heart rate variability standards of measurement, physiological interpretation, and clinical use. Eur Heart J 17, 354-381 (1996).

81. Matthews, B. W. Comparison of the predicted and observed secondary structure of T4 phage lysozyme. Biochim Biophys Acta Protein Struct 405, 442-451 (1975).

82. Baldi, P., Brunak, S., Chauvin, Y., Andersen, C. A. F. \& Nielsen, H. Assessing the accuracy of prediction algorithms for classification: an overview. Bioinformatics 16, 412-424 (2000).

83. Jensen, A., Hohmann, M. \& Künzel, W. Dynamic changes in organ blood flow and oxygen consumption during acute asphyxia in fetal sheep. J Dev Physiol 9, 543-559 (1987).

84. Preziosi, M. P., Roig, J. C., Hargrove, N. \& Burchfield, D. J. Metabolic acidemia with hypoxia attenuates the hemodynamic responses to epinephrine during resuscitation in lambs. Crit Care Med 21, 1901-1907 (1993).

85. Executive summary: Neonatal encephalopathy and neurologic outcome, second edition. Report of the American College of Obstetricians and Gynecologists' Task Force on Neonatal Encephalopathy. Obs Gynecol 123, 896-901 (2014).

86. Parer, J. T., King, T., Flanders, S., Fox, M. \& Kilpatrick, S. J. Fetal acidemia and electronic fetal heart rate patterns: Is there evidence of an association? J Matern Neonatal Med 19, 289-294 (2006).

87. George, S. A. et al. Fetal heart rate variability and brain stem injury after asphyxia in preterm fetal sheep. Am J Physiol Regul Integr Comp Physiol 287, R925-R933 (2004).

88. Van Laar, J. O. E. H., Porath, M. M., Peters, C. H. L. \& Oei, S. G. Spectral analysis of fetal heart rate variability for fetal surveillance: review of the literature. Acta Obstet Gynecol Scand 87, 300-306 (2008).

89. Westgate, J. A., Bennet, L. \& Gunn, A. J. The role of fetal ECG monitoring in labour. Fetal Matern Med Rev 13, 119-139 (2002). 
90. Strachan, B. K., Van Wijngaarden, W. J., Sahota, D. S., Chang, A. \& James, D. K. Cardiotocography only versus cardiotocography plus PR-interval analysis in intrapartum surveillance: a randomised, multicentre trial. Lancet 355, 456-459 (2000).

91. Rosén, K. G., Dagbjartsson, A., Henriksson, B. A., Lagercrantz, H. \& Kjellmer, I. The relationship between circulating catecholamines and ST waveform in the fetal lamb electrocardiogram during hypoxia. Am J Obstet Gynecol 149, 190-195 (1984).

92. Algra, A., Roelandt, J. R., Tijssen, J. G., Simoons, M. L. \& Pool, J. Effect of beta-blockers on the relation between QT-interval and heart rate in exercise ECG. Eur Heart J 8 Suppl D, 71-73 (1987).

93. Abildskov, J. A. Adrenergic effects of the QT interval of the electrocardiogram. Am Heart J 92, 210-216 (1976).

94. Lommen, C. M. L. et al. An algorithm for the automatic detection of seizures in neonatal amplitudeintegrated EEG. Acta Paediatr 96, 674-680 (2007).

95. Stockard-Pope, J. E., Werner, S. S. \& Bickford, R. G. Atlas of Neonatal Electroencephalography. (Raven Press, 1992).

96. Barlow, R. M. The foetal sheep: morphogenesis of the nervous system and histochemical aspects of myelination. J Comp Neurol 135, 249-261 (1969).

97. McIntosh, G. H., Baghurst, K. I., Potter, B. J. \& Hetzel, B. S. Foetal brain development in the sheep. Neuropathol Appl Neurobiol 5, 103-114 (1979).

98. Davidson, J. O., Quaedackers, J. S. L. T., George, S. A., Gunn, A. J. \& Bennet, L. Maternal dexamethasone and EEG hyperactivity in preterm fetal sheep. J Physio/ 589, 3823-3835 (2011).

99. Bennet, L. et al. The effect of cerebral hypothermia on white and grey matter injury induced by severe hypoxia in preterm fetal sheep. J Physiol 578, 491-506 (2007).

100. Esteller, R., Echauz, J., Tcheng, T., Litt, B. \& Pless, B. Line length: an efficient feature for seizure onset detection. in 2001 Conference Proceedings of the 23rd Annual International Conference of the IEEE Engineering in Medicine and Biology Society 2, 1707-1710 (IEEE, 2001).

101. Greene, B. R., Marnane, W. P., Lightbody, G., Reilly, R. B. \& Boylan, G. B. Classifier models and architectures for EEG-based neonatal seizure detection. Physiol Meas 29, 1157-1178 (2008).

102. D’Alessandro, M. et al. Epileptic seizure prediction using hybrid feature selection over multiple intracranial EEG electrode contacts: a report of four patients. IEEE Trans Biomed Eng 50, 603-615 (2003).

103. Richman, J. S. \& Moorman, J. R. Physiological time-series analysis using approximate entropy and sample entropy. Am J Physiol Heart Circ Physiol 278, H2039-H2049 (2000).

104. Faul, S. Automated neonatal seizure detection. (University College Cork - National University of Ireland, 2007).

105. De Weerd, A. W., Despland, P. A. \& Plouin, P. Neonatal EEG. The International Federation of Clinical Neurophysiology. Electroencephalogr Clin Neurophysiol Suppl 52, 149-157 (1999).

106. Kohavi, R. A Study of Cross-Validation and Bootstrap for Accuracy Estimation and Model Selection. Int Jt Conf Artif Intell 14, 1137-1143 (1995).

107. Chang, C. C. \& Lin, C. J. LIBSVM: a library for support vector machines. ACM Trans Intell Syst Technol 2, 27 (2011).

108. Luts, J. et al. A tutorial on support vector machine-based methods for classification problems in chemometrics. Anal Chim Acta 665, 129-145 (2010).

109. Platt, J. Probabilistic outputs for support vector machines and comparisons to regularized likelihood methods. Adv Large Margin Classif 10, 61-74 (1999).

110. Lin, H. T., Lin, C. J. \& Weng, R. C. A note on Platt's probabilistic outputs for support vector machines. Mach Learn 68, 267-276 (2007). 
111. Görges, M., Markewitz, B. A. \& Westenskow, D. R. Improving alarm performance in the medical intensive care unit using delays and clinical context. Anesth Analg 108, 1546-1552 (2009).

112. Temko, A., Thomas, E. M., Marnane, W. P., Lightbody, G. \& Boylan, G. B. Performance assessment for EEG-based neonatal seizure detectors. Clin Neurophysiol 122, 474-482 (2011).

113. Demšar, J. Statistical comparisons of classifiers over multiple data sets. J Mach Learn Res 7, 1-30 (2006).

114. Shellhaas, R. A., Soaita, A. I. \& Clancy, R. R. Sensitivity of amplitude-integrated electroencephalography for neonatal seizure detection. Pediatrics 120, 770-777 (2007).

115. Shah, D. K. et al. Accuracy of bedside electroencephalographic monitoring in comparison with simultaneous continuous conventional electroencephalography for seizure detection in term infants. Pediatrics 121, 1146-1154 (2008).

116. Van Rooij, L. G. M., De Vries, L. S., Van Huffelen, A. C. \& Toet, M. C. Additional value of two-channel amplitude integrated EEG recording in full-term infants with unilateral brain injury. Arch Dis Child Fetal Neonatal Ed 95, F160-F168 (2010).

117. Toet, M. C., Van der Meij, W., De Vries, L. S., Uiterwaal, C. S. P. M. \& Van Huffelen, K. C. Comparison between simultaneously recorded amplitude integrated electroencephalogram (cerebral function monitor) and standard electroencephalogram in neonates. Pediatrics 109, 772-779 (2002).

118. Shellhaas, R. A. \& Clancy, R. R. Characterization of neonatal seizures by conventional EEG and singlechannel EEG. Clin Neurophysiol 118, 2156-2161 (2007).

119. Kotsiantis, S. B. Emerging Artificial Intelligence Applications in Computer Engineering: Real World Al Systems with Applications in eHealth, $\mathrm{HCl}$, Information Retrieval and Pervasive Technologies. (IOS Press, 2007).

120. De Vos, M. et al. Automated artifact removal as preprocessing refines neonatal seizure detection. Clin Neurophysiol 122, 2345-2354 (2011).

121. Biagioni, E. et al. Background EEG activity in preterm infants: Correlation of outcome with selected maturational features. Electroencephalogr Clin Neurophysiol 91, 154-162 (1994).

122. Patrizi, S., Holmes, G. L., Orzalesi, M. \& Allemand, F. Neonatal seizures: Characteristics of EEG ictal activity in preterm and fullterm infants. Brain Dev 25, 427-437 (2003).

123. Lombroso, C. T. Quantified electrographic scales on 10 pre-term healthy newborns followed up to 40-43 weeks of conceptional age by serial polygraphic recordings. Electroencephalogr Clin Neurophysiol 46, 460-474 (1979).

124. Bayley, N. Bayley scales of infant development: manual. (Psychological Corporation, 1993).

125. Niemarkt, H. J. et al. Maturational changes in automated EEG spectral power analysis in preterm infants. Pediatr Res 70, 529-534 (2011).

126. Tekgul, H., Bourgeois, B. F., Gauvreau, K. \& Bergin, A. M. Electroencephalography in neonatal seizures: comparison of a reduced and a full 10/20 montage. Pediatr Neurol 32, 155-161 (2005).

127. Jennekens, W. et al. Automatic burst detection for the EEG of the preterm infant. Physiol Meas 32, 162337 (2011).

128. Conde, J. R. C. et al. Extrauterine life duration and ontogenic EEG parameters in preterm newborns with and without major ultrasound brain lesions. Clin Neurophysiol 116, 2796-2809 (2005).

129. Niemarkt, H. J. et al. Quantitative analysis of maturational changes in EEG background activity in very preterm infants with a normal neurodevelopment at 1year of age. Early Hum Dev 86, 219-224 (2010).

130. Scher, M. S., Johnson, M. W., Ludington, S. M. \& Loparo, K. A. Physiologic brain dysmaturity in late preterm infants. Pediatr Res 70, 524-528 (2011).

131. Sisman, J., Campbell, D. E. \& Brion, L. P. Amplitude-integrated EEG in preterm infants: maturation of background pattern and amplitude voltage with postmenstrual age and gestational age. J Perinatol 25, 391-396 (2005). 
132. Volpe, J. J. Brain injury in the premature infant. Neuropathology, clinical aspects, pathogenesis, and prevention. Clin Perinatol 24, 567-587 (1997).

133. Nelson, K. B. \& Leviton, A. How much of neonatal encephalopathy is due to birth asphyxia? Am J Dis Child 145, 1325-1331 (1991).

134. Liem, K. D. \& Greisen, G. Monitoring of cerebral haemodynamics in newborn infants. Early Hum Dev 86, 155-158 (2010).

135. Pryds, O. \& Edwards, A. D. Cerebral blood flow in the newborn infant. Arch Dis Child Fetal Neonatal Ed 74, F63-9 (1996).

136. Penáz, J., Honziková, N. \& Fiser, B. Spectral analysis of resting variability of some circulatory parameters in man. Physiol Bohemoslov 27, 349-357 (1978).

137. Akselrod, S. et al. Hemodynamic regulation: investigation by spectral analysis. Am J Physiol 249, H867H875 (1985).

138. De Boer, R. W., Karemaker, J. M. \& Strackee, J. Hemodynamic fluctuations and baroreflex sensitivity in humans: a beat-to-beat model. Am J Physiol 253, H680-H689 (1987).

139. Ursino, M. \& Magosso, E. Role of short-term cardiovascular regulation in heart period variability: a modeling study. Am J Physiol Heart Circ Physiol 284, H1479-93 (2003).

140. Laude, D. et al. Comparison of various techniques used to estimate spontaneous baroreflex sensitivity (the EuroBaVar study). Am J Physiol Regul Integr Comp Physiol 286, R226-R231 (2004).

141. La Rovere, M. T., Pinna, G. D. \& Raczak, G. Baroreflex sensitivity: measurement and clinical implications. Ann Noninvasive Electrocardiol 13, 191-207 (2008).

142. Andriessen, P. et al. Baroreceptor reflex sensitivity in human neonates: the effect of postmenstrual age. J Physiol 568, 333-341 (2005).

143. Jennekens, W., Dat, M., Bovendeerd, P. H. M., Wijn, P. F. F. \& Andriessen, P. Validation of a preterm infant cardiovascular system model under baroreflex control with heart rate and blood pressure data. Conf Proc IEEE Eng Med Biol Soc 2011, 896-899 (2011).

144. Shinebourne, E. A., Vapaavuori, E. K., Williams, R. L., Heymann, M. A. \& Rudolph, A. M. Development of baroreflex activity in unanesthetized fetal and neonatal lambs. Circ Res 31, 710-718 (1972).

145. Maloney, J. E., Cannata, J., Dowling, M. H., Else, W. \& Ritchie, B. Baroreflex activity in conscious fetal and newborn lambs. Biol Neonate 31, 340-350 (1977).

146. Frasch, M. G. et al. Fetal body weight and the development of the control of the cardiovascular system in fetal sheep. J Physiol 579, 893-907 (2007).

147. Booth, L. C. et al. Is baroreflex control of sympathetic activity and heart rate active in the preterm fetal sheep? Am J Physiol Regul Integr Comp Physiol 296, R603-R609 (2009).

148. Bennet, L., Rossenrode, S., Gunning, M. I., Gluckman, P. D. \& Gunn, A. J. The cardiovascular and cerebrovascular responses of the immature fetal sheep to acute umbilical cord occlusion. $J$ Physiol 517, 247-257 (1999).

149. Robbe, H. W. et al. Assessment of baroreceptor reflex sensitivity by means of spectral analysis. Hypertension 10, 538-543 (1987).

150. Andriessen, P. et al. Cardiovascular fluctuations and transfer function analysis in stable preterm infants. Pediatr Res 53, 89-97 (2003).

151. Bernardi, L. et al. New method to measure and improve consistency of baroreflex sensitivity values. Clin Auton Res 20, 353-361 (2010).

152. Jensen, A. \& Hanson, M. A. Circulatory responses to acute asphyxia in intact and chemodenervated fetal sheep near term. Reprod Fertil Dev 7, 1351-1359 (1995). 
153. Frasch, M. G., Muller, T., Szynkaruk, M. \& Schwab, M. Validation of spontaneous assessment of baroreceptor reflex sensitivity and its relation to heart rate variability in the ovine fetus pre- and near-term. Can J Physiol Pharmacol 87, 736-742 (2009).

154. Suzuki, H., Sugawara, J., Kimura, Y., Murakami, T. \& Okamura, K. Contribution of the fetal baroreceptor reflex to the low frequency component of fetal heart rate fluctuations. Gynecol Obstet Invest 55, 156-161 (2003).

155. Sagawa, K. in Comprehensive Physiology (ed. Terjung, R. L.) 453-496 (John Wiley \& Sons, Ltd, 2011).

156. Roelants-van Rijn, A. M. et al. Neonatal diffusion-weighted MR imaging: relation with histopathology or follow-up MR examination. Neuropediatrics 32, 286-294 (2001).

157. Ikeda, T. et al. Fetal heart rate patterns in postasphyxiated fetal lambs with brain damage. Am J Obstet Gynecol 179, 1329-1337 (1998).

158. Blanco, C. E., Dawes, G. S., Hanson, M. A. \& McCooke, H. B. Carotid baroreceptors in fetal and newborn sheep. Pediatr Res 24, 342-346 (1988).

159. Segar, J. L., Hajduczok, G., Smith, B. A., Merrill, D. C. \& Robillard, J. E. Ontogeny of baroreflex control of renal sympathetic nerve activity and heart rate. Am J Physio/ 263, H1819-H1826 (1992).

160. Segar, J. L. Ontogeny of the arterial and cardiopulmonary baroreflex during fetal and postnatal life. Am J Physiol 273, R457-R471 (1997).

161. Booth, L. C. et al. Baroreflex control of renal sympathetic nerve activity and heart rate in near-term fetal sheep. Exp Physiol 96, 736-744 (2011).

162. Persson, P. B. et al. Time versus frequency domain techniques for assessing baroreflex sensitivity. $J$ Hypertens 19, 1699-1705 (2001).

163. Matsuda, Y., Maeda, T. \& Kouno, S. The critical period of non-reassuring fetal heart rate patterns in preterm gestation. Eur J Obstet Gynecol Reprod Biol 106, 36-39 (2003).

164. Williams, K. P. \& Galerneau, F. Intrapartum fetal heart rate patterns in the prediction of neonatal acidemia. Am J Obstet Gynecol 188, 820-823 (2003).

165. Nelson, K. B., Dambrosia, J. M., Ting, T. Y. \& Grether, J. K. Uncertain value of electronic fetal monitoring in predicting cerebral palsy. N Engl J Med 334, 613-618 (1996).

166. Struijk, P. C. et al. Blood pressure estimation in the human fetal descending aorta. Ultrasound Obstet Gynecol 32, 673-681 (2008).

167. Kovacsovics-Bankowski, M. et al. Pre-clinical safety testing supporting clinical use of allogeneic multipotent adult progenitor cells. Cytotherapy 10, 730-742 (2008).

168. Jacobs, S. A. et al. Human multipotent adult progenitor cells are nonimmunogenic and exert potent immunomodulatory effects on alloreactive T-cell responses. Cell Transplant 22, 1915-1928 (2013).

169. Jacobs, S. A., Roobrouck, V. D., Verfaillie, C. M. \& Van Gool, S. W. Immunological characteristics of human mesenchymal stem cells and multipotent adult progenitor cells. Immunol Cell Biol 91, 32-39 (2013).

170. Busch, S. A. et al. Multipotent adult progenitor cells prevent macrophage-mediated axonal dieback and promote regrowth after spinal cord injury. J Neurosci 31, 944-953 (2011).

171. Bedi, S. S. et al. Intravenous multipotent adult progenitor cell therapy attenuates activated microglial/macrophage response and improves spatial learning after traumatic brain injury. Stem Cells Transl Med 2, 953-60 (2013).

172. Fischer, U. M. et al. Pulmonary passage is a major obstacle for intravenous stem cell delivery: the pulmonary first-pass effect. Stem Cells Dev 18, 683-692 (2009).

173. Back, S. A., Riddle, A. \& Hohimer, A. R. Role of instrumented fetal sheep preparations in defining the pathogenesis of human periventricular white-matter injury. J Child Neurol 21, 582-589 (2006).

174. Niemarkt, H. J. et al. Multi-channel amplitude-integrated EEG characteristics in preterm infants with a normal neurodevelopment at two years of corrected age. Early Hum Dev 88, 209-216 (2012). 
175. Zwanenburg, A. et al. Heart rate-mediated blood pressure control in preterm fetal sheep under normal and hypoxic-ischemic conditions. Pediatr Res 73, 420-426 (2013).

176. Boozer, S. et al. Global Characterization and Genomic Stability of Human MultiStem, A Multipotent Adult Progenitor Cell. J Stem Cells 4, 17-28 (2009).

177. Gelman, A. et al. Bayesian data analysis. (CRC Press, Taylor \& Francis Group, LLC, 2014).

178. Miller, S. P. et al. Seizure-associated brain injury in term newborns with perinatal asphyxia. Neurology $\mathbf{5 8}$, 542-548 (2002).

179. Gluckman, P. D. et al. Selective head cooling with mild systemic hypothermia after neonatal encephalopathy: Multicentre randomised trial. Lancet 365, 663-670 (2005).

180. Glass, H. C. et al. Clinical Neonatal Seizures are Independently Associated with Outcome in Infants at Risk for Hypoxic-Ischemic Brain Injury. J Pediatr 155, 318-323 (2009).

181. De Vries, L. S. \& Toet, M. C. Amplitude Integrated Electroencephalography in the Full-Term Newborn. Clinics in Perinatology 33, 619-632 (2006).

182. Fyfe, K. L., Yiallourou, S. R., Wong, F. Y. \& Horne, R. S. C. The development of cardiovascular and cerebral vascular control in preterm infants. Sleep Medicine Reviews 18, 299-310 (2014).

183. Mays, R. W. et al. Development of an allogeneic adherent stem cell therapy for treatment of ischemic stroke. J Exp Stroke Transl Med 3, 34-46 (2010).

184. Lehman, N. et al. Development of a surrogate angiogenic potency assay for clinical-grade stem cell production. Cytotherapy 14, 994-1004 (2012).

185. Mallard, C. et al. Astrocytes and microglia in acute cerebral injury underlying cerebral palsy associated with preterm birth. Pediatr Res 1-7 (2013). doi:10.1038/pr.2013.188

186. Voulgari-Kokota, A. et al. Mesenchymal stem cells protect CNS neurons against glutamate excitotoxicity by inhibiting glutamate receptor expression and function. Exp Neurol 236, 161-170 (2012).

187. Offner, H. et al. Experimental stroke induces massive, rapid activation of the peripheral immune system. $J$ Cereb blood flow Metab 26, 654-665 (2006).

188. Offner, H. et al. Splenic atrophy in experimental stroke is accompanied by increased regulatory $\mathrm{T}$ cells and circulating macrophages. J Immunol 176, 6523-6531 (2006).

189. Ajmo, C. T. et al. The spleen contributes to stroke-induced neurodegeneration. J Neurosci Res 86, 22272234 (2008).

190. Liesz, A. et al. Regulatory T cells are key cerebroprotective immunomodulators in acute experimental stroke. Nat Med 15, 192-199 (2009).

191. Glezer, I., Simard, A. R. \& Rivest, S. Neuroprotective role of the innate immune system by microglia. Neuroscience 147, 867-883 (2007).

192. Miron, V. E. et al. M2 microglia and macrophages drive oligodendrocyte differentiation during CNS remyelination. Nat Neurosci 16, 1211-8 (2013).

193. Gelman, A., Jakulin, A., Pittau, M. G. \& Su, Y.-S. A weakly informative default prior distribution for logistic and other regression models. Ann Appl Stat 2, 1360-1383 (2008).

194. Silva, I. et al. Noninvasive fetal ECG: The PhysioNet/Computing in Cardiology Challenge 2013. in Computing in Cardiology Conference (CinC), 2013 29, 149-152 (2013).

195. Clifford, G. D., Silva, I., Behar, J. \& Moody, G. B. Non-invasive fetal ECG analysis. Physiol Meas 35, 1521 (2014).

196. Vullings, R. et al. A robust physiology-based source separation method for QRS detection in low amplitude fetal ECG recordings. Physiol Meas 31, 935-951 (2010).

197. Abdel-Aleem, S., St. Louis, J. D., Hughes, G. C. \& Lowe, J. E. Metabolic changes in the normal and hypoxic neonatal myocardium. Ann N Y Acad Sci 874, 254-261 (1999). 
198. Shellhaas, R. A., Gallagher, P. R. \& Clancy, R. R. Assessment of Neonatal Electroencephalography (EEG) Background by Conventional and Two Amplitude-Integrated EEG Classification Systems. J Pediatr 153, 369-374 (2008).

199. Kostović, I. \& Jovanov-Milošević, N. The development of cerebral connections during the first 20-45 weeks' gestation. Semin Fetal Neonatal Med 11, 415-422 (2006).

200. Kostović, I. \& Judaš, M. The development of the subplate and thalamocortical connections in the human foetal brain. Acta Paediatr 99, 1119-1127 (2010).

201. Fransson, P., Åden, U., Blennow, M. \& Lagercrantz, H. The functional architecture of the infant brain as revealed by resting-state fMRI. Cereb Cortex 21, 145-154 (2011).

202. Paul, L. K. Developmental malformation of the corpus callosum: A review of typical callosal development and examples of developmental disorders with callosal involvement. J Neurodev Disord 3, 3-27 (2011).

203. Meijer, E. J. et al. Functional connectivity in preterm infants derived from EEG coherence analysis. Eur J Paediatr Neurol 18, 780-9 (2014).

204. Koolen, N. et al. Development of an Interhemispheric Symmetry Measurement in the Neonatal Brain. in Proceedings - International Conference on Pattern Recognition Application and Methods 765-770 (2014).

205. Räsänen, O., Metsäranta, M. \& Vanhatalo, S. Development of a novel robust measure for interhemispheric synchrony in the neonatal EEG: Activation Synchrony Index (ASI). Neuroimage 69, 256-266 (2013).

206. Koolen, N. et al. Interhemispheric synchrony in the neonatal EEG revisited: activation synchrony index as a promising classifier. Front Hum Neurosci 8, 1030 (2014).

207. Liu, A. J. et al. Arterial baroreflex function is an important determinant of acute cerebral ischemia in rats with middle cerebral artery occlusion. Life Sci 83, 388-393 (2008).

208. Victor, R. G. Carotid baroreflex activation therapy for resistant hypertension. Nat Rev Cardiol 1-13 (2015). doi:10.1038/nrcardio.2015.96

209. Domingos, P. A few useful things to know about machine learning. Commun ACM 55, 78 (2012).

210. Greene, B. R. et al. A comparison of quantitative EEG features for neonatal seizure detection. Clin Neurophysiol 119, 1248-1261 (2008). 
Summary 

The personal and societal burden of hypoxic-ischemic encephalopathy (HIE) is immense. HIE is expressed as persistent brain injury induced by severe perinatal hypoxia (low blood oxygen levels) and/or ischemia (severely reduced blood flow). Brain injury occurs in two stages. The first stage is acute and takes place during hypoxic-ischemic conditions. The second stage of brain injury starts several hours after hypoxia-ischemia has been resolved. Infants with HIE will often experience lifelong effects, e.g. motor and learning disabilities, cerebral palsy, and blindness. Neonatal encephalopathy globally leads to 50 million disability-adjusted life-years (DALY). In comparison, the entire spectrum of cancer was estimated to lead to 169 million DALY globally. Though part of the burden can be reduced through general improvements to obstetric care in regions where public health care is less developed, additional reduction can only be realised through new and innovative improvements in perinatal care. Computer-assisted analysis has the potential of aiding physicians in assessing complex and challenging physiological signals, thus improving patient care. In this thesis we focussed on computer-assisted signal analysis in the two stages of HIE-related brain injury with the aim of improving the diagnostic tools available to physicians.

First, more accurate and earlier detection of conditions leading to the first stage of brain injury is essential to prevent or limit the extent of HIE. Fetal ECG contains characteristics that can be used to assess the hypoxic and acidotic conditions during birth that might lead to HIE. Heart rate and changes in the ST waveform of the fetal ECG have been implemented in clinical devices as markers to assess the fetal metabolic compromise related to severe hypoxia. Recent meta-analyses of clinical trials demonstrate that aforementioned devices effect little-if any-improvement in primary outcomes, e.g. a reduction in HIE. The cause for these findings is unknown. We hypothesised that these findings might be due to the choice of markers used in the devices. Several ECG based markers were reported in literature. We compared these markers within a fetal ovine model for standardized severe hypoxia-ischemia to identify which of these markers might be suitable for assessment of fetal hypoxia and acidosis. We found that the length of ventricular activation and repolarisation time, as well as heart rate may be useful interval-based markers for assessing fetal hypoxia. In contrast, waveform analysis of the ST segment was not useful as an early marker of fetal hypoxia. When compared to interval-based ECG markers, waveform-based markers are less reliable due to dependency on ECG lead and heart vector orientation. We found similar results with respect to ST waveform analysis using clinical Neoventa STAN S31 device in the fetal ovine model. Despite the basic observation that the ST waveform does change during prolonged hypoxia, the device was unable to adequately provide alarms. We speculated that this was mostly due to algorithm formulation.

Secondly, we investigated several markers on their suitability to assist clinicians in making treatment decisions for infants that potentially develop the second stage of brain injury. Currently, treatment of HIE needs to start before the onset of the second stage of brain injury for it to be effective. This means that assessment and treatment prognosis is required before the second stage begins. One issue here is that current markers that would enable physicians to reliably base their treatment decisions on are only effective after onset of the second phase of brain injury. Identification of suitable markers for basing outcome prognosis on is thus necessary. Beyond prognosis, the ability to monitor the effect of HIE and treatment on the functional level may help 
physicians make further decisions. In this thesis we investigated markers that are related to cortical, subcortical and autonomic function, and could be used for monitoring purposes and/or prognostic purposes.

In hypoxic-ischemic encephalopathy seizures not only indicate cortical dysfunction due to neuronal damage in hypoxic-ischemic encephalopathy, but they also may contribute to further brain injury. In preterm infants, seizures are often of short duration ( $<1 \mathrm{~min}$ ) and difficult to detect using existing seizure detection algorithms. We developed and evaluated a method that facilitated detection of short seizures in preterm infants by assessing the transition to and from seizure state in the EEG. This method can be used to provide improved detection of seizures and thus affects clinical treatment decisions.

Interhemispheric synchrony of EEG burst activity may be used as a marker for interhemispheric neuronal connectivity and subcortical function. In literature qualitative decreases of interhemispheric neuronal activity in HIE have been reported. Knowledge on baseline values for interhemispheric connectivity in healthy individuals is essential to allow quantitative descriptions of changes therein. Up to now, baseline values for interhemispheric burst synchrony in healthy preterm infants are not well established. Though various, slightly different methods enable manual estimation of interhemispheric burst synchrony, this estimation is a laborious task and subject to error. We developed an algorithm that enabled automated calculation of interhemispheric EEG burst synchrony. Using the algorithm we demonstrated a high degree of interhemispheric burst synchrony in healthy preterm infants (28-36 weeks postmenstrual age) that was not related to postmenstrual age. This finding indicates the early fetal presence of functional cortico-cortical (e.g. corpus callosum) and thalamocortical connections. The algorithm facilitates further quantitative investigation of interhemispheric burst synchrony in HIE.

Baroreceptor reflex associated cardiovascular fluctuations were assessed as marker for autonomic function. The baroreceptor reflex is a regulatory mechanism that maintains arterial blood pressure at a relatively constant level. Disruption of baroreceptor reflex function exposes the brain to periods of hypertension and hypotension. Disruption of baroreceptor reflex function thus threatens the still maturing vasculature of the infant brain and limits sufficient cerebral perfusion. HIE clearly attenuated operation of the baroreceptor reflex. However, baroreceptor reflex function was preserved after stem cell treatment. Hence the baroreceptor reflex may be used as a marker for identifying HIE treatment effects. 
Valorisation 

The personal and societal burden of hypoxic-ischemic encephalopathy (HIE) is immense. Infants with HIE will often experience lifelong effects, e.g. motor and learning disabilities, cerebral palsy, and blindness. Neonatal encephalopathy globally leads to 50 million disability-adjusted life-years (DALY). In comparison, the entire spectrum of cancer was estimated to lead to 169 million DALY globally. Reducing incidence and/or severity of HIE decreases the healthcare and personal costs involved in providing lifelong treatment and support.

This thesis advocates the use of computer-assisted analysis to allow physicians to make a more accurate decision on intervention and treatment. The aim is to use computer algorithms to provide timely and non-redundant information about the fetus or infant that is sensitive and specific to the issue being investigated. In this thesis, such information was based on electro-physiological signals. In chapter two, for example, ECG signal characteristics were used as markers for fetal metabolic compromise that would necessitate immediate intervention.

The design of clinical devices for fetal monitoring is in general based on relatively simple principles of preset threshold values. Alarms are generated after breaching thresholds. In general, threshold values for alarm limits are determined empirically, using a relatively small data set for training and algorithm validation. This approach works to some degree, but using small data sets increases the risk of overfitting. Overfitting means that the algorithm is too specifically designed for the data available for training and evaluation. Due to overfitting, an algorithm will perform less well when analysing new and unseen data. For example we discovered in our analysis that the Neoventa STAN S31 device used for ST waveform analysis did not take into account the negative changes in T/QRS ratio. We speculate that the devices' algorithm to assess only positive T/QRS ratio changes was likely because the data reported by the developers of the algorithm did not show negative T/QRS ratio changes as a response to fetal metabolic compromise. Negative T/QRS ratio changes were, however, present within our data set. Consequentially, the device did not perform well in our investigation. The same issue may also be a contributing factor to the lack of improvement in primary outcome by ST waveform analysis that was cited in the meta-analyses referred to in chapter two. Resources went into developing a device that, after expensive clinical trials, did not seem to yield the improvement expected by both the developers and clinicians, and public money was also invested in purchasing said devices for clinical use. Choices made in algorithm design have both healthcare and economic implications. This means that the decision making process that leads to the final algorithm and the data set on which it is tested need to be carefully evaluated to avoid accidental oversights.

One way to make algorithm development and design less prone to overfitting and other biases is to rely on machine learning methods and to use representative clinical data sets.

Machine learning algorithms can relate a multitude of markers - features with a numerical input value-to an outcome value. An example would be to relate fetal heart rate, fetal heart variability and other fetal ECG markers to metabolic compromise as an outcome. Machine learning algorithms need to know how to relate features to an outcome, i.e. the algorithm needs to 'learn' a model. To do so, such an algorithm is trained using a data set for which the outcome is known. An example was 
demonstrated in chapter 4, where a machine learning algorithm was trained to relate several EEG-derived features to annotated seizures. The next step after training is to evaluate how well the algorithm can use the model for predicting outcome. This requires the use of data other than that of the training set. If the training set were also to be used for validation, the resulting performance would be inflated due to overfitting. By repeating training and validation using different data each time it is possible to arrive at a relatively unbiased estimate of true performance. Naturally, using an entirely new data set each time training and validation is repeated is often not possible. Luckily, it is also not necessary as various methods exist to randomly divide a data set into training and validation sets.

There is also the issue of the representativeness of the data set used. The data set used for development of the Neoventa ST waveform analysis algorithm seems not to be representative because it lacked negative T/QRS changes in response to hypoxia and acidosis. Machine learning would not have prevented the issue with negative T/QRS changes even though it might have yielded different alarm thresholds for T/QRS changes. The data sets for this dissertation consisted of measurements in an ovine preterm model (chapters 2-4, 6-7) and in human infants (chapter 5). The ovine model we used had the broad advantage of reproducibility of HIE and the ability to do histological investigations. The original experiments with the ovine model were designed tot investigate on the one hand the processes involved in HIE and on the other hand potential HIE treatments. The ovine model also had specific advantages and limitations for the purpose of devising algorithm parameters. The main advantage was that measurements were reproducible, that hypoxia-ischemia led to a severe degree of $\mathrm{HIE}$, and that data was measured continuously. These qualities are necessary and sufficient for assessing the usefulness of markers for the first and second stages of brain injury in HIE. The limitation of the ovine model is that results, e.g. threshold values for markers, cannot be directly translated from bench-to-bedside. The processes leading to HIE in clinical practice are more diverse than the hypoxia-ischemia induced by the temporary complete umbilical cord occlusion of our model. Also, while the values of physiological parameters in the ovine fetus are comparable to human fetus or infant in range and in responses to hypoxia and acidosis, they are not the same.

We expect that a major improvement in medical algorithm development in obstetrics and neonatology can be made if a large database of human perinatal physiological signals can be constructed. This database can be used to accurately train and validate algorithms for HIE and other neonatal diseases and disorders. The data to be measured and included in the database should include fetal ECG and fetal blood gas analyses before birth, and at least ECG, EEG (reduced 10/20 system), blood pressure, body temperature, and blood gas analysis after birth. A challenge in developing the database will be the fact that despite the major consequences of neonatal encephalopathy on the global scale, the actual occurrence is relatively low at an estimated 1.5 cases per 1000 live births. Hence a large number of recordings has to be made to capture a reasonable cross-section of clinically occurring HIE. Such a database was unavailable for this thesis. Setting it up will require not only the efforts of a large group of gynaecologists and neonatologists, but also the support of experts in advanced signal analysis and machine learning. 
The algorithms developed and described in chapters four and five for calculating marker values can be directly used for further investigations. The algorithm described in chapter four is a novel approach to use temporal information in subsequently measured values of features or markers in a machine learning algorithm. Such an approach is especially useful if the event to be identified has a short temporal duration and cannot be identified based solely on feature values at each separate time point.

The algorithm on burst synchrony investigation as described in chapter five can be directly implemented into EEG monitoring devices, albeit not in devices intended for amplitude-integrated EEG monitoring. The latter devices commonly use only one or two EEG channels, which does not provide the electrode coverage required. It should be noted that reduction in interhemispheric burst synchrony in HIE has, to our knowledge, only been described qualitatively. Our algorithm allows quantitative investigation of burst synchrony as well. 



\section{Acknowledgements}



Most science is evolutionary, not revolutionary. This thesis is no different. It should be seen as a contribution, albeit humble, to the scientific discourse on computers in medicine in general and within perinatal and neonatal care in particular. For such a work I am heavily indebted to many colleagues and friends, some of which I will mention here.

First of all, I would like to extend my gratitude to my promotors, prof. dr. Tammo Delhaas and prof. dr. Boris Kramer, and my co-promotors, dr. Peter Andriessen and dr. Tim Wolfs. They helped tremendously in writing - and rewriting - the article manuscripts which constitute the main body of this thesis. Through our interactions I learned a great deal about myself and proper academic research in clinical settings.

Naturally, I would also like to thank my co-workers, dr. Reint Jellema and Daan Ophelders of the department of Pediatrics for managing our joint animal experiments and for having the patience to hear me out and understand my view on things. I would also like to thank Ben Hermans, who, as a Master's student, collaborated in part of my research projects.

Of course, a pleasant working environment is an important source of motivation. I'd like to thank my colleagues and the students at the biomedical engineering lab, Wouter Donders, Bart Spronck, Yvette Klinkenberg-Koeken, Georgina Palau Caballero, Jeire Steinbuch, Marieke Lijnkamp-Pluijmert, Lauren Dupuis, John Walmsley, Sjeng Quicken, Peter Huntjens, Kujtim Gashi, Tamara Klopmeijer and Maarten Heusinkveld, for creating and maintaining a good atmosphere in the lab and for the occasional serenade. I found the department of biomedical engineering to be a very welcoming group. The interactions between the different postdoc researchers and $\mathrm{PhD}$ candidates broadened my perspective on the field of cardiovascular modelling. I wish dr. Joost Lumens, dr. Koen Reesink and dr. Wouter Huberts good luck with their research lines.

I was happy to receive technical support at both the department of biomedical engineering and the department of pediatrics, and would like to thank Jeroen Hameleers, Nico Kloosterboer, Lilian Kessels and Leon Janssen for their technical assistance.

Let me also thank Claire Meertens, office manager of the BME department, without whom my academic life would have been a lot more difficult.

Last, but not least, I would like to thank my dear friends and my parents for their continued support and my now-wife for her support during the final stages of thesis writing. 



\section{Curriculum vitae \& publication list}





\section{Curriculum Vitae}

Alexander Adriaan Zwanenburg was born on the 6th of February 1985 in Rotterdam. That day and the days that followed seem to have been rather frosty, and an Elfstedentocht was organised later that month. Not that it mattered to young Alex, as he was kept warm in an incubator at the nursery of a local hospital. Decades would pass before he would visit a nursery again. As he grew older, his natural inquisitiveness and his continuous search for intellectual challenges led him to start his Bachelor's in applied physics in 2003. Illness and a year serving as a board member of the study association for applied physics at Eindhoven University of Technology led him to complete his Bachelor's study in 2010. He had started his Master's studies in applied physics before that. His internships took him back to the hospital setting. First he was an intern at the radiotherapy department of the Catharina Hospital in Eindhoven, before continuing to the medical physics department at the Máxima Medical Centre in Veldhoven to write his Master's thesis. During this time he worked with the departments of pediatrics of the hospital and of Maastricht University. After receiving his Master's degree in 2011, he was invited to continue his work as a PhD student under promotors prof. dr. Tammo Delhaas and prof. dr. Boris Kramer and co-promotors dr. Peter Andriessen and dr. Tim Wolfs. This resulted in the thesis you, dear reader, are reading this very moment. After his PhD studies, Alex will continue his career as a postdoc in radiomics at the OncoRay Center for Radiation Research in Oncology in Dresden.

\section{Publication list}

Jellema, R. K., Ophelders, D. R. M. G., Zwanenburg, A., Nikiforou, M., Delhaas, T., Andriessen, P., Mays, R. W., Deans, R. W., Germeraad, W. T. V., Wolfs, T. G. A. M. \& Kramer, B. W. Clinical-grade multipotent adult progenitor cells for hypoxicischemic injury in the preterm brain. Submitted to J Neuroinflammation (2015)

Seehase, M., Jennekens, W., Zwanenburg, A., Andriessen, P., Collins, J. J. P, Kuypers, E., Zimmermann, L. J., Vles, J. S. H., Gavilanes, A. W. D.,\& Kramer, B.W. Propofol administration to the maternal-fetal unit improved fetal EEG and influenced cerebral apoptotic pathway in preterm lambs suffering from severe asphyxia. Mol Cell Pediatr 2, 4 (2015)

Zwanenburg, A., Andriessen, P., Jellema, R. K., Niemarkt, H. J., Wolfs, T. G. A. M., Kramer, B. W. \& Delhaas, T. Using trend templated in a neonatal seizure algorithm improves detection of short seizures in a fetal ovine model. Physiol Meas 36, 369-384 (2015)

Meijer, E. J., Hermans, K. H., Zwanenburg, A., Jennekens, W., Niemarkt, H. J., Cluitmans, P. J., Van Pul, C., Wijn, P. F. F. \& Andriessen, P. Functional connectivity in preterm infants derived from EEG coherence analysis. Eur J Paediatr Neurol 18, 780-789 (2014) 
Jellema, R. K., Lima Passos, V., Ophelders, D. R. M. G., Wolfs, T. G., Zwanenburg, A., De Munter, S., Nikiforou, M., Collins, J. J. P., Kuypers, E., Bos, G. M., Steinbusch, H. W., Vanderlocht, J., Andriessen, P., Germeraad, W. T. \& Kramer, B. W. Systemic GCSF attenuates cerebral inflammation and hypomyelination but does not reduce seizure burden in preterm sheep exposed to global hypoxia-ischemia. J Exp Neurol 250, 293-303 (2013)

Zwanenburg, A., Jellema, R. K., Jennekens, W., Ophelders, D. R. M. G., Vullings, R., Van Hunnik, A., Van Pul, C., Bennet, L., Delhaas, T., Kramer, B. W. \& Andriessen, P. Heart rate mediated blood pressure control in preterm fetal sheep under normal and hypoxicischemic conditions. Ped Res 73, 420-426 (2013)

Jellema, R. K., Lima Passos, V., Zwanenburg, A., Ophelders, D. R. M. G., De Munter, S., Vanderlocht, J., Germeraad, W. T., Kuypers, E., Collins, J. J. P., Cleutjens, J. P., Jennekens, W., Gavilanes, A. W. D., Seehase, M., Vles, H. J., Steinbusch, H., Andriessen, P., Wolfs, T. G. A. M. \& Kramer, B. W. Cerebral inflammation and mobilization of the peripheral immune system following global hypoxia-ischemia in preterm sheep. J Neuroinflammation 10, 13

Jellema, R. K., Wolfs, T. G. A. M., Lima Passos, V., Zwanenburg, A., Ophelders, D. R. M. G., Kuypers, E., Hopman, A. H., Dudink, J., Steinbusch, H. W., Andriessen, P., Germeraad, W. T., Vanderlocht, J. \& Kramer, B. W. Mesenchymal stem cells induce T-cell tolerance and protect the preterm brain after global hypoxiaischemia. PloS one 8, e73031 (2013)

Zwanenburg, A., Meijer E., Jennekens, W., Van Pul, C., Kramer, B. W. \& Andriessen, P. Automatic dectection of burst synchrony in preterm infants. Conf Proc IEEE Eng Med Biol Soc, 4720-4723 (2012) 


\section{Propositions}



1. Developers of clinical algorithms should draw upon machine learning techniques and representative databases for algorithm development.

2. Interval-based ECG markers are more robust to inter-subject variation in heart position and instrumentation then waveform-based ECG markers.

3. Taking development of EEG signal characteristics over time into account aids in detecting seizures that would otherwise be missed.

4. Heart rate mediated baroreceptor reflex function is negatively affected by hypoxic-ischemic encephalopathy.

5. Alarm fatigue is at least as dangerous to patient health as a lack of alarms.

6. Given the increasing role of technology in clinical practice, medical students should be taught the fundamentals of medical technology.

7. Any system that on close inspection might appear chaotic can behave orderly when viewed from a distance.

8. Computer assisted analysis of electro-physiological signals improves assessment of an infant's condition by physicians. 